University of Louisville

ThinkIR: The University of Louisville's Institutional Repository

Electronic Theses and Dissertations

7-2006

\title{
Software based deployment of encryption keys in wireless sensor networks.
}

Timothy William Hnat

University of Louisville

Follow this and additional works at: https://ir.library.louisville.edu/etd

\section{Recommended Citation}

Hnat, Timothy William, "Software based deployment of encryption keys in wireless sensor networks."

(2006). Electronic Theses and Dissertations. Paper 622.

https://doi.org/10.18297/etd/622

This Master's Thesis is brought to you for free and open access by ThinkIR: The University of Louisville's Institutional Repository. It has been accepted for inclusion in Electronic Theses and Dissertations by an authorized administrator of ThinkIR: The University of Louisville's Institutional Repository. This title appears here courtesy of the author, who has retained all other copyrights. For more information, please contact thinkir@louisville.edu. 


\title{
SOFTWARE BASED DEPLOYMENT OF ENCRYPTION KEYS IN WIRELESS SENSOR NETWORKS
}

\author{
By \\ Timothy William Hnat \\ M.Eng., University of Louisville, 2006
}

\begin{abstract}
A Thesis
Submitted to the Faculty of the University of Louisville Speed School of Engineering as Partial Fulfillment of the Requirements for the Professional Degree of
\end{abstract}

\section{MASTER OF ENGINEERING}

Department of Computer Engineering and Computer Science

July 2006 


\title{
SOFTWARE BASED DEPLOYMENT OF ENCRYPTION KEYS IN WIRELESS SENSOR NETWORKS
}

\author{
Submitted by \\ Timothy William Hnat
}

A Thesis Approved on

by the Following Reading and Examination Committee:

Rammohan K. Ragade, Thesis Advisor

Adel S. Elmaghraby

John F. Naber 


\begin{abstract}
Sensor networks are just in their infancy. Their use will continue to grow as the technology becomes cheaper and more efficient. A current shortcoming with sensor networks is the inability to efficiently provide secure communications. As sensor networks are deployed to monitor and control systems, the security of communications will become a more important.

This thesis proposes a new approach to key establishment and renewal through the use of point-to-point keys and software verification and validation to ensure the integrity of two nodes. Sensor networks exist on limited resources, so power efficiency is a concern. The proposed protocol allows for the use of small keys instead of large pre-distributed keys.

This thesis explores the design and implementation of a new point-to-point key generation and renewal algorithm. The main contribution is the development of an algorithm that utilizes a software integrity check to ensure the validity of a node. The thesis also utilizes a simulated sensor network to test and validate the new software algorithm.
\end{abstract}




\section{ACKNOWLEDGMENTS}

I would like to express my appreciation to my advisor, Dr. Rammohan Ragade, for his support and guidance during my pursuit of this research and my committee, Dr. Adel Elmaghraby and Dr. John Naber. I would like to thank Jeff Hieb and Brian Carter who have helped me through the research process and have provided insight into the technical side of sensors and security. I would also like to thank my parents, William and Janice Hnat, for supporting me through these past 5 years of college. 


\section{TABLE OF CONTENTS}

\section{CHAPTER}

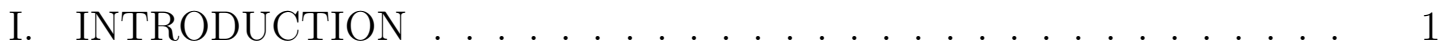

1.1. Background . . . . . . . . . . . . . . . . 1

1.2. Organization of Thesis . . . . . . . . . . . . . . 4

II. LITERATURE REVIEW . . . . . . . . . . . . . . . . . . . 5

2.1. Cryptographic Keys . . . . . . . . . . . . . . . . . . 10

2.2. Authentication ................... 14

2.3. Availability . . . . . . . . . . . . . . . . . . 14

2.3.1. Sybil Attack . . . . . . . . . . . . 15

2.4. Scalability . . . . . . . . . . . . . 16

2.5. Efficient Cryptographic Systems . . . . . . . . . . . . . 16

2.6. Attacks . . . . . . . . . . . . . . . 16

III. THEORY AND DESIGN . . . . . . . . . . . . . . . . . . . . 19

3.1. Wireless Sensors . . . . . . . . . . . . . . . . . . 19

3.2. TinyOS Framework . . . . . . . . . . . . . . 20

3.3. TOSSIM . . . . . . . . . . . . . . . . . 21

3.4. Energy Consumption . . . . . . . . . . . . . . . . . 22

3.5. Design of Algorithms . . . . . . . . . . . . . . . . 22

3.5.1. Session Keys . . . . . . . . . . . . . . . . 23

3.5.2. Soft Key Distribution _. . . . . . . . . . . . . 23

IV. IMPLEMENTATION . . . . . . . . . . . . . . . . . . . . . 29

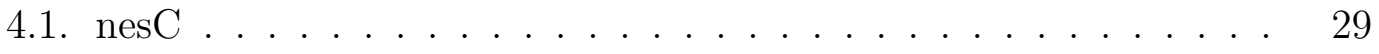

4.2. Application Code . . . . . . . . . . . . . . . . 30 
V. TESTING AND RESULTS . . . . . . . . . . . . . . 33

5.1. Testing Overview . . . . . . . . . . . . . . . . . 33

5.1.1. Testing Key Generation . . . . . . . . . . . . . . . . . . 33

5.1.2. Testing Network Communication . . . . . . . . . . . . 34

5.2. Experimental Design . . . . . . . . . . . . . . 37

5.2.1. Sample Tree Application . . . . . . . . . . . . . . 37

5.3. Security . . . . . . . . . . . . . . . . . 42

5.3.1. Intrusion . . . . . . . . . . . . . . . 42

5.3.2. Breaking the Encryption . . . . . . . . . . . 43

5.3.3. Misuse or Abuse . . . . . . . . . . . . . . . 46

VI. CONCLUSIONS AND FUTURE DIRECTIONS . . . . . . . . . 48

6.1. Conclusions . . . . . . . . . . . . . . . . . . . . 48

6.2. Future Directions . . . . . . . . . . . . . . . 50

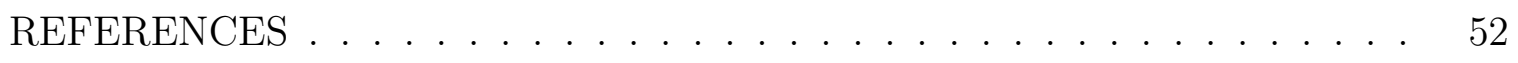
APPENDIX

I. TinyOS System . . . . . . . . . . . . . . . . . . . 58

1.1. TinyOS Installation $\ldots \ldots \ldots \ldots$

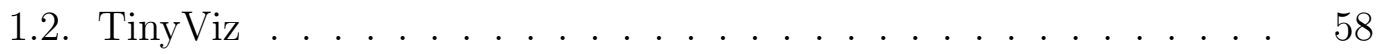

1.3. Power Profiling . . . . . . . . . . . . . . . 59

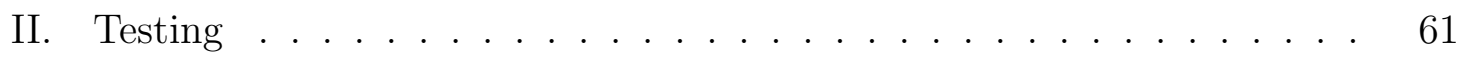

2.1. Key Generation Testing . . . . . . . . . . . . . . . . 61

2.2. Network Communication Testing . . . . . . . . . . . 63

2.3. Sample Tree Application Test . . . . . . . . . . . . . . 64

2.4. Base Tree Power Profile . . . . . . . . . . . . . . . 71

2.5. New Tree Power Profile . . . . . . . . . . . . . . . 73

III. Source Code . . . . . . . . . . . . . . . . . . . . . . 76

3.1. Network Test Application . . . . . . . . . . . . 76 
3.1.1. NetTest.nc . . . . . . . . . . . . . . . . . 76

3.1.2. NetTestM.nc . . . . . . . . . . . . . . . 76

3.2. Basic Tree Application . . . . . . . . . . . . . . . . . 77

3.2.1. Treenc ......................... 78

3.2.2. TreeM.nc ..................... . . 78

3.3. Encrypted Tree Application . . . . . . . . . . . . . . . 80

3.3.1. TreeTest.nc . . . . . . . . . . . . . . . . . 80

3.3.2. TreeTestM.nc . . . . . . . . . . . . . . 80 


\section{LIST OF TABLES}

TABLE 2.1. Benefits and drawbacks of key distribution schemes . . . . . . . 14

TABLE 5.1. Simulation Results for Key Generation Testing . . . . . . . . . 35

TABLE 5.2. Simulation Results for Network Communications Testing . . . . 36 


\section{LIST OF FIGURES}

FIGURE 2.1. Wireless Network Example . . . . . . . . . . . . . . 6

FIGURE 2.2. Compromised Master Key Example . . . . . . . . . . . . . . . . 7

FIGURE 2.3. Compromised Group Key Example . . . . . . . . . . . . . . . . 7

FIGURE 2.4. Compromised Point-to-Point Key Example . . . . . . . . . . . . 8

FIGURE 3.1. Mica2 mote . . . . . . . . . . . . . . . . . . . 20

FIGURE 3.2. XBow Sensor Board . . . . . . . . . . . . . . . . . . . 21

FIGURE 3.3. Setup Key Procedure . . . . . . . . . . . . . . . . . . . . 24

FIGURE 3.4. Validation Request Procedure . . . . . . . . . . . . . . . 25

FIGURE 3.5. Example Memory Map . . . . . . . . . . . . . . . . . . 26

FIGURE 3.6. Algorithm Message Passing . . . . . . . . . . . . . . . . . . 27

FIGURE 4.1. TOS_Msg Structure (from AM.h) . . . . . . . . . . . . . . . 31

FIGURE 4.2. Int_Msg Structure . . . . . . . . . . . . . . . . . 31

FIGURE 5.1. Key Generation Test Setup . . . . . . . . . . . . . . . . 34

FIGURE 5.2. Network Communications Test Setup . . . . . . . . . . . . 36

FIGURE 5.3. TOSSIM Network Test . . . . . . . . . . . . . . . . 37

FIGURE 5.4. Basic Tree Routing . . . . . . . . . . . . . . . . . . 38

FIGURE 5.5. Basic Tree Power Chart . . . . . . . . . . . . . . . . . . 39

FIGURE 5.6. Encrypted Tree Power Chart . . . . . . . . . . . . . . . . 40

FIGURE 5.7. Difference Power Chart . . . . . . . . . . . . . . . . . 40

FIGURE 5.8. Power Efficiency of the Algorithm . . . . . . . . . . . . . 41

FIGURE 5.9. Key Renewal Algorithm . . . . . . . . . . . . . . . . . . 44 


\section{CHAPTER I INTRODUCTION}

\subsection{Background}

Wireless sensor networks are becoming popular as a net method to gather information. The miniaturization of digital electronics, with regards to chip and sensor design, are allowing wireless sensor grids to become an economically practical solution.

Early work in sensor technologies used large wired sensors to gather data on large remote devices to monitor from afar. However, the technology today allows sensors to be battery powered and manufacturing costs are low enough to place multiple sensors within a given area. A device is created that can monitor and report information to a central location by reducing the cost per sensor and making their communication wireless. "A sensor grid is comprised of many individual and independent sensors which can be deployed inside or near a phenomenon" [1].

Wireless grids can be utilized for a variety of scenarios. Many sensors monitor temperature, humidity, lighting conditions, pressure, noise levels, chemical makeup of the area, etc... The sensors can be utilized for military applications from personnel

detection to battlefield surveillance and chemical attack detection [43, 11]. Other uses for sensor technology include monitoring human health and the environment $[43,11,28,29]$. For the consumer, home automation and smart environments require the abilities of the sensor grid to gather and distribute information. Commercial impacts involve better control of environmental conditions in office buildings [1]. 
The new wireless sensor grids present a whole new set of problems including, but not limited to: device integrity, communication security, and operating lifetime (battery power). As the devices become smaller, more power efficient, and less expensive, limitations are placed on the ability to secure each device and to maintain the security of network communications. A problem possible in hostile situations can be the enemy, with more sensors, has a larger opportunity to capture and/or break into the communication network of sensors $[43,15,20]$.

A lack of organization is inherent to the nature of wireless grids. Each sensor does not know where it will end up and with whom it can safely communicate. It is not aware of the location of other sensors within the grid. The sensor knows of nearby sensors but not their locations in the environment. This lack of organization presents problems in resolving communication channels back to a master controller, as well as problems with trust among other devices in the grid.

A critical component that must be addressed for large scale deployment of wireless sensor grids is the cost of the system. At present, each wireless sensor node costs upward of 250 dollars, a price which is cost prohibitive for wide-scale deployment [10]. The cost needs to be under one dollar per sensor for the economics of wireless grids to make fiscal sense [1]. At that price, sensors are expendable, something which is necessary in battlefield scenarios.

The power consumption of these wireless sensors is still too large to be of use in long-term environments and scenarios. The advantage of wireless sensor grids is limited due to the inability to provide long-term monitoring capabilities. Power consumption needs to be reduced and power sources must be improved. This could involve alternative power from solar and other environmental sources to support batteries. The long term capabilities need to rely on a power source that is available for extended periods of time. Without a long term wireless sensor, the network is neither fiscally nor logistically feasible to maintain $[1,35]$. 
These sensors are usually built upon a framework package known as TinyOS [1]. This framework provides the ability to quickly program and control sensor packages without the need to custom program each device during assembly. These sensors are built upon an Atmel micro-controller and run the AVR instruction set. TinyOS is capable of running on other types of sensors. TinyOS wraps this in a framework to provide a quick and easy interface for the sensors to work with.

Communication between sensors in the grid environment is cause for concern in security oriented deployments [1]. Basic communication protocols broadcast the transmitted information in a ASCII stream. The thrust of research up to this point has been on getting sensor networks functional and scaleable, not in the security of their information. This problem of scalability has been addressed in a variety of methods. These methods are based in software and network routing schemes.

Basic communication protocols and transmission methods utilize an omnidirectional antenna to both send and receive signals, though this does not provide any type of security in the transmission. However, directional antennas can limit the signal broadcast to the direction of the sensor in which communication is going to take place. The complexity of a directional approach makes this technique infeasible for most scenarios.

Wireless sensor grids are currently receiving a lot of research attention; however, the majority of research examines the communication and handoff protocols or the implementation side of networks. This thesis will describe the design and implementation of a software authentication procedure for establishing unique security keys between sensor nodes. 


\subsection{Organization of Thesis}

Chapter II presents a detailed literature review of wireless sensor grid concepts, techniques, and concerns. Chapter III discusses the design of the software based key determination approach. Chapter IV describes the implementation of the softwarebased key determination algorithm. Chapter V contains the testing and results from both simulation of the wireless grid system and a real-world scenario. Chapter VI contains conclusions and future directions. 


\section{CHAPTER II LITERATURE REVIEW}

Sensor grids are deployed for a wide variety of uses. A potential application area of concern is hostility to the grid infrastructure [32, 43, 15]. Sensor grids will be subjected to various security attacks within the confines of their deployment [32]. Three issues must be addressed with the deployments of sensors: (1) attacks on communications and data integrity from the sensors, (2) the physical vulnerability associated with deployed sensors, and (3) a method to provide a "self-checking" aspect within the sensor network and on the sensors themselves [32].

In order to verify that the program residing within a sensor has not been modified (integrity), some sort of authentication mechanism must be utilized [32]. One method is the use of a Program-Integrity Verification (PIV) server. A controller on a wireless grid is able to maintain a list of all authenticated nodes [32]. This process involves checking and authenticating the nodes as they join the network or after experiencing a long service blockage. This verification process does contain overhead. However, this overhead can be made relatively small as compared to traditional cryptographic functions. The verification process can utilize a cryptographic hash function that is "orders-of-magnitude cheaper and faster than other approaches" [32]. The hash functions can be optimized for the embedded controllers at both the 8- and 16-bit sizes.

The system architecture plays a major role in how resilient to attack the net-

work and individual nodes can be made. Nodes can be made relatively secure through a variety of well-defined hardware design methods. There are two general classes of hardware security mechanisms: read-proof and tamper-proof. Read-proof hardware 


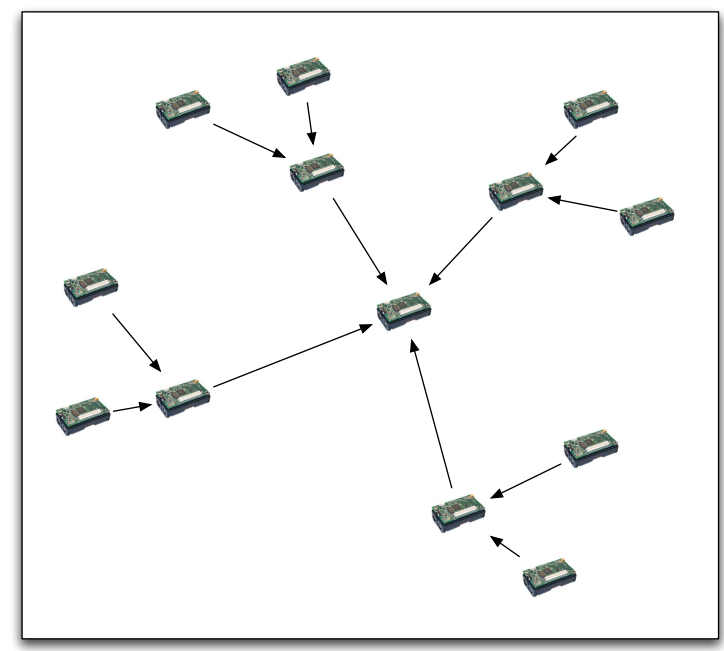

FIGURE 2.1 - Wireless Network Example

are the group of technologies that prevent the enemy from reading data contained within the chip. Tamper-proof hardware are the group of technologies that prevent the enemy from changing data on the chip [18]. This technology, so far, is relatively expensive to produce and cost prohibitive for deployment in sensor networks [33]. How the wireless system is setup in regards to the architecture will affect its security and resiliency.

Figure 2.1 demonstrates an example of a wireless sensor network. The solid arrows denote secure wireless traffic. A breakdown of a master key encrypted network, as shown in figure 2.2, has all of its communications insecure. Once a master key is discovered, the entire network is lost. The utilization of a group-based key system prevents the entire network from being lost (Figure 2.3). The best method is to use a point-to-point key system so if a link or node is compromised, only the current node is lost and not the rest of the network (Figure 2.4).

Attacks on a wireless grid come from many sources. "Node compromise is the central problem that uniquely characterizes the sensor network's threat model" $[35,21]$. The first is a hostile attack on the transmitted signal. This involves capturing some of the wireless signal data and processing it in an attempt to break into 


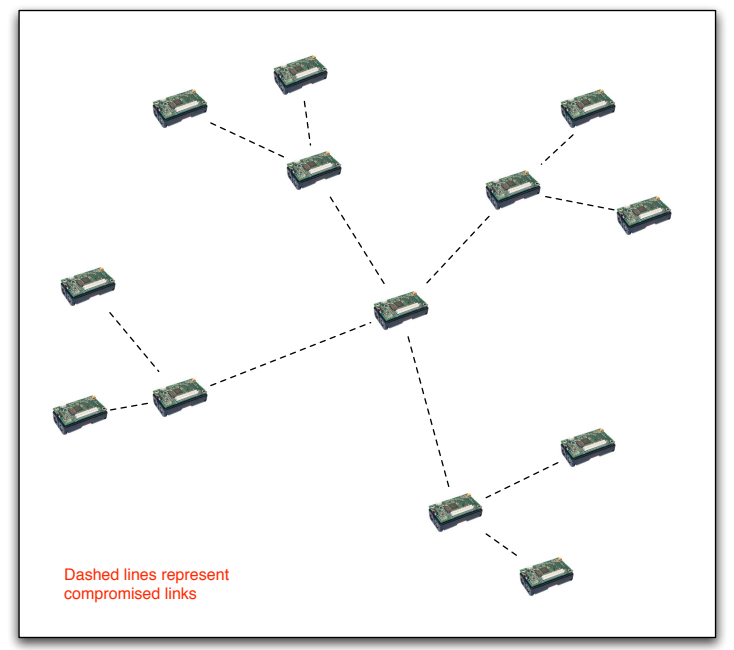

FIGURE 2.2-Compromised Master Key Example

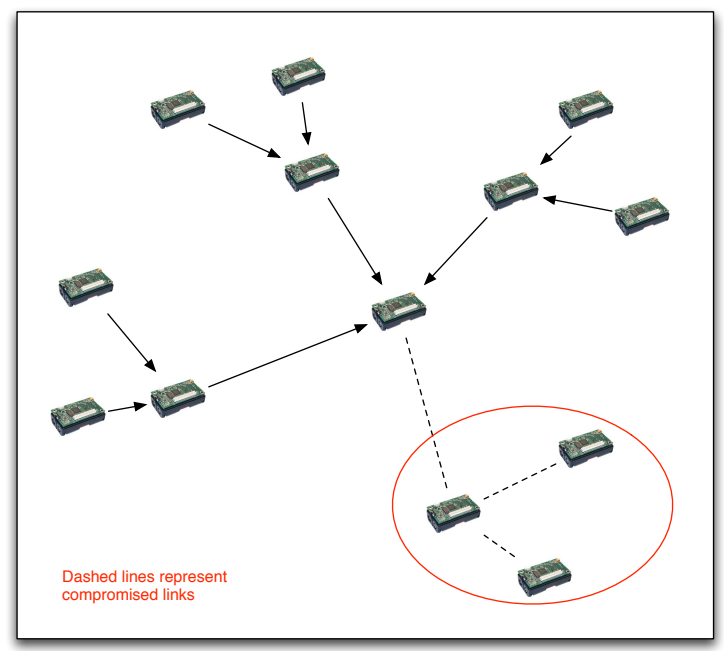

FIGURE 2.3-Compromised Group Key Example 


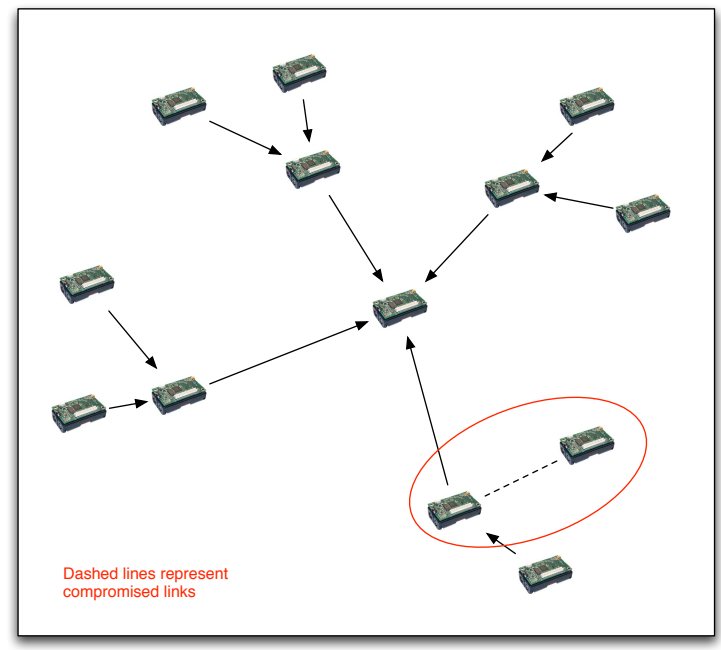

FIGURE 2.4-Compromised Point-to-Point Key Example

the current encryption of the wireless network. "Sensor nodes use wireless communication, which is particularly easy to eavesdrop on" $[35,9,11]$. This is impossible to detect from within the network. Passive monitoring will not cause any interference to the network.

The second attack vector is a physical attack on the node. This involves obtaining a node from the network or a replica of one and taking it into a lab to use a more powerful computer to break into the system. In the worst case, a scanning electron microscope could be utilized to dissect the memory from the device and rebuild the system on a new device, though this would be a very expensive process and most likely take too much time to be of much use.

A third option for attack would be to cause service disruptions to the wireless grid. This could be as easy as jamming the wireless signal or doing any thing else that would disrupt the ability of the network to do its task. Nodes could be destroyed in an attempt to decrease the range of the network. Once the network has been compromised, a data attack can be made on the system. Compromised nodes could be made to send invalid data throughout the network in an effort to disrupt the ability of the sensing grid $[31,16,35,33]$. 
The last attack vector is a more traditional method from the world of network computing. The wireless sensors are battery-powered devices and because of this, severely constrained in resources and lifetime. A resource consumption attack would attempt to get the individual nodes to utilize their sensors, processor, or transmitter in an effort to consume available battery resources [3]. This would quickly end the life of the sensor and eliminate it from the system. This also parallels denial-of-service attacks as seen on traditional networks [32, 35, 33, 11].

The ultimate problem with any wireless sensor network is the ability of a foreign body to capture and reproduce nodes in a more sophisticated device with more memory in such a way that the new device functions the same way from network's perspective as the original node but contains malicious code $[32,11]$. In traditional computing and security, physical security is one of the primary foci and communication restrictions another. A generally accepted practice is once an intruder gains physical access to a machine, anything on that machine is no longer secure and safe.

Security within wireless sensor grids needs to be able to scale efficiently to large-scale deployments $[35,2,6,20]$. The mechanisms and protocols need to have either bandwidth efficiency or be self-contained within a cluster of sensor nodes. This will allow for large-scale deployments to occur.

Sensor networks are expected to grow to many thousands of nodes in the future. This large scale deployment presents a scalability problem that is similar to problems occurring in large scale super-computing. The main difference is the computational and bandwidth resources available to the sensor networks. Security is usually not of too much concern within the super-computing environment other than at the perimeter of the system. The security model for sensor networks needs to be ultra scaleable for any viable deployment $[35,33,2,9,6]$. 


\subsection{Cryptographic Keys}

The security requirements for a key distribution protocol involve the availability of sensor nodes, authenticating sensor nodes, integrity of communication messages, confidentiality of communication, non-reputation of nodes, survivability of sensor nodes, and degradation of security services $[4,19]$. All of these are critical to ensure that total security of the system is kept intact. Key distribution mechanisms involve the ability to scale the key scheme to large networks, the efficiency at which this distribution occurs is measured in storage, processing and communication complex-

ity, key connectivity and resilience to capture $[4,15,19,28]$. The key distribution schemes involve one of two types of techniques: pre-distribution or post-distribution schemes.

The management systems need to provide and ensure key independence (if the protocol allows this), perfect forward secrecy, and backward/forward secrecy [2]. The key independence guarantees that an adversary, knowing a subset of the keys, cannot discover future or prior keys. "Forward secrecy guarantees that a passive adversary who knows a subset of old group keys cannot discover subsequent group keys, while backward secrecy guarantees that a passive adversary who knows a subset of group keys cannot discover preceding group keys" [2].

Key distribution within wireless sensor networks is critical to the ability of the system to keep communications secure. Many different approaches exist in this field. The pair-wise key pre-distribution scheme involves computing distinct pair-wise keys to distribute to the nodes [13]. The base case is typically only to utilize a single master key and distribute this to all nodes. This is a significant problem when one of the nodes is captured and the entire network is compromised. The best case is to generate n-1 keys for the network of size $n$ in order to keep communication between all nodes open. This poses problems with the scalability of the network [4, 8, 27, 40].

The random pair-wise key scheme addresses the storage component of the 
basic scheme at the expense of the inter-node key connectivity $[12,4,13]$. This scheme loads a small subset of the total keys into each node in an attempt to provide a high probability of matching a fellow node's key. This scheme has the same benefits and weaknesses as the basic key distribution scheme mentioned with regards to node compromise and scalability. The only benefit is with the storage of keys within each node $[8,34]$.

The closest pair-wise key pre-distribution scheme provides a more efficient system as compared to the random or basic schemes; however, this scheme relies on the ability to know approximately where each sensor is going to be placed [4, 25, 27]. This is not a good technique for networks in which sensors are not going to be placed in specific areas [14]. The ID-based one-way function scheme provides a mechanism in which two nodes can share a key in, at most, two hops [4]. Multiple ID based oneway function schemes offer a more efficient approach to the single one-way function scheme in terms of memory usage. The disadvantage of these schemes is that with a compromised node, multiple nodes within the network will now be compromised [12, 4, 27].

The broadcast session key negotiation protocol is based upon the master key pre-distribution solution. Each sensor receives a key prior to deployment and all other keys are based upon this key. A compromised master key results in a completely compromised network. The lightweight key management system is a modification of the broadcast session protocol. The lightweight system utilizes multiple groups (generations) in which each generation contains a unique key. These generations vary in size from entire networks to small subsets of nodes [4].

The random keychain-based key pre-distribution solution is based upon a probabilistic key scheme. This involves generating a large key-space and randomly drawing keys without replacement for use within the sensor networks. Probability is used such that sensors must have at least one key pair in common with each other and have to 
search through their list to obtain this information $[4,7]$. The difficult part about this scheme is in what constraints to make on key generation and distribution to ensure a secure and reliable network.

The key-chains that are utilized in the random key-chain scheme are determined at runtime. The idea behind combinatorial design-based key pre-distribution schemes is to design the key-chains using combinatorial design theory. This ensures that sensor nodes will find exactly one common key and the probability of the network communication succeeding is 100 percent. Scalability becomes a problem in computing these key-chains for utilization [4].

In a key matrix based dynamic key generation solution, Blom's scheme utilized two $\mathrm{N}$ x N matrices. There are public and private key matrices. The disadvantage to this method is that all sensor nodes within a given radio range must respond with a computation of their two matrices. This is a significant overhead for the computational resources on nodes. The multiple space key pre-distribution scheme utilized multiple private matrices and a shared key discovery scheme. This eliminates some of the computation and communication overhead [4].

The polynomial based dynamic key generation scheme utilized a method to symmetric polynomial distribution for key generation. Each pair of sensor nodes can generate their unique key from initial information common to both. The location of the sensor node (if known) can be utilized to more effectively compute shared keys $[14,21,24,25]$. This approach also is in utilization of a shared key discovery algorithm [4].

The lightweight key management system is a group-wise key distribution scheme. This utilizes a pair-wise key scheme that can be distributed to groups of sensors as they are deployed in different phases. The group-wise keys are distributed through the already secured pair-wise keys among sensors [4].

Network-wise key distribution schemes rely on a wireless sensor network to be 
based upon a tiered security level. For a master key solution, the base station can broadcast a network-wise key to the nodes. A better approach is to use a multi-tiered solution in which nodes are broken up into different security levels. The more sensitive the information, the more secure the node is required to be. The problem with both solutions is that once the master/network keys are compromised, the entire security level is compromised $[4,35]$.

Public key cryptography is difficult to implement within sensor networks. The computational overhead for generation and validation of keys is more than the sensor's processors can handle $[6,14,26,37,38,40]$. One approach is to move the computationally expensive operations for the public key system to external components of the sensor networks. This is only possible when these resources exist [39]. This approach depends on extra resources that may not be available in all sensor networks. Another approach is allowing the network to manage and create the trust relations required for public key cryptography [5].

An alternative method of implementing public key cryptography is to include a custom hardware chip to offload the computational overhead of the public key system from the small processor [17]. This would allow the public key computations to occur more quickly and consume much less power. "The implementation of NtruEncrypt consumed less than $20 \mu \mathrm{W} "$ [17]. This is significantly better than a general purpose processor could hope for.

An alternative to basic public key cryptography is to utilize the elliptic curve cryptography [38]. This has been difficult to implement because of the time and resources required to generate the keys. It has been shown that it is possible to implement elliptic curve cryptography on the sensor nodes and have "reasonable" results [30]. The definition of reasonable is left to the system designer and is dependent on the conditions the network is being designed for. 


\begin{tabular}{|c|c|c|c|}
\hline & Benefits & Drawbacks & Key Compromise \\
\hline Pair-wise key & Complete communication & Can not scale & Entire network \\
\hline Random Pair-wise & Communication & Can not scale well & Almost entire network \\
\hline Closest Pair-wise & Efficient & Requires sensor locations & Compromised area \\
\hline ID-based & Low memory usage for keys & Compromised Nodes & Multiple nodes \\
\hline Broadcast Session & Keys can be changed & Utilizes a master key & Entire network \\
\hline Random Keychain & Space efficient & Scalability & Portions of the network \\
\hline Group-wise & Better Scalability & Requires sensor locations & Groups of nodes \\
\hline Public Key & Secure & Requires computation power & Groups of nodes \\
\hline Point-to-Point Key & Scalable & Overhead to create keys & Captured node links \\
\hline
\end{tabular}

TABLE 2.1

Benefits and drawbacks of key distribution schemes

\subsection{Authentication}

Authentication is a component of the overall security of a sensor network and is necessary to allow sensors to verify and check the packets for malicious activity, which ensures the integrity of data $[35,20]$. However, authentication can only go so far in protecting the network. A compromised node will be able to inject invalid data into the stream and still pass the authentication checks. This integrity ensures protection against man-in-the-middle attacks [42].

\subsection{Availability}

A sensor network must maintain availability. The entire goal of the network is to monitor the area and report its information back to a specified location. If the availability is broken, part of the network will be unable to perform this task. A Denial-of-Service (DOS) attack is the most common vector in which to attack the 
availability of the sensor network. An important feature of the network is to provide a graceful degradation as nodes are either compromised or reach the end of their operating life [35, 9, 11, 20, 28].

Availability is a real concern for applications of sensor networks within disaster areas, for utilization with public safety, and in home healthcare $[41,28,29]$. Each of these three areas present their own concerns for security and availability of the network. In the disaster area, the network will likely be subjected to harsh environment and the system needs to ensure availability and robustness. The system needs to provide information as long as possible with a significant number of nodes ceasing to function. This robustness should provide the availability for the network [41]. In the case of public safety, the networks would be monitoring areas for hazardous material. Availability is crucial to not miss anything. An attack on this kind of network could be cause to look for a real threat $[41,28]$. The home healthcare environment has a necessity to provide high availability and confidential networks. These networks could provide vital signs and accident-notifications to the authorized users [41, 28, 29].

\subsubsection{Sybil Attack}

A common attack to sensor network availability is called the Sybil attack. This is an attack where a malicious node illegitimately claims multiple identities [35, 31]. This attack can occur at multiple logical layers. An attack to the routing of the network can alter the paths packets will take. By assuming multiple identities, a node can attempt to get more than its share of packets to come to it and selectively forward packets [31]. The Sybil attack can also be utilized at the Media Access Control (MAC) layer to attempt to consume all the available shared communication bandwidth. This has the effect of causing the real nodes to be unable to transmit their information $[35,31]$. 


\subsection{Scalability}

Scalability is a critical problem for sensor networks. As most security protocols have been designed for node to base station security, this is a problem for massive (thousands) of sensor nodes operating within a single mesh network [35, 9, 6, 40]. Scalability is necessary in order for the networks to be successful. A point-to-point network which is arranged into groups of sensors will provide a very scalable system. Each node will only be concerned with communicating among its nearby neighbors and not with the entire network.

\subsection{Efficient Cryptographic Systems}

Sensor nodes are severely constrained in regards to their processor and power capabilities. This is a problem for traditional security measures due to the fact that many require a large computational capability to accomplish their tasks. There seems to be a direct correlation between the capabilities of the cryptographic protocol and the amount of power required to accomplish this encryption [35].

\subsection{Attacks}

A multitude of attack vectors exist for wireless sensor networks. Outsider attacks require robustness and strength of the protocols to provide security against passive monitoring of the communication channels. The outsider attack can also be by packet injection. Packet injection involves inserting incorrect data into an existing network. This attack can be prevented through the use of a large quantity of sensor nodes and placing the nodes in such a way that their coverage areas overlap. Overlap allows the sensors to verify the information provided from other locations in the network [35]. Insider attacks are much more difficult to create; however, if successful they should be virtually undetectable. In order to cause an insider attack, a sensor 
must be compromised through an attack sufficient to allows modification of the sensor functionality without showing this modification to the sensor network. One method is to physically capture a node and interface it with a more powerful computer. This computer will be able to utilize the sensor, in its original state, to insert the data it wishes into the network [35].

DOS is an effective vector with which to attack a sensor network. Sensor networks rely on wireless communications and low power system. A DOS attack on the wireless communications will leave little bandwidth for utilization by legitimate traffic. Jamming of the signal involves transmitting noise on the frequencies used within the sensor network for communication. This will prevent the nodes from communicating and cause DOS to occur. This technique is effective only within the area the jammer can transmit. This will stop an entire network, given a large enough deployment area. A slightly more effective method is to only transmit data to cause packet collisions. It is not necessary to jam the signal all of the time. Constant jamming will alert the network to the problem. Selective collisions of wireless packets is a more directed attack and should eliminate a selected area of the network [41].

Sensor network, unlike traditional wired networks like the Internet or local area networks (LAN), rely on their batteries for operation. Attacking this power source is a method to eliminate the sensor network. A DOS attack, using directed packet injection, aimed at the sensor nodes could cause them to waste their power receiving and examining the packets. Any other method of causing the sensor to waste power should be just as effective at attacking the sensors [41].

The prior two attacks relied on brute-force attacks to the network. A more power efficient and effective strategy would be to selectively target and attack the network. A homing attack is a method of DOS that relies on traffic observance prior to commencing the attack. By observing the traffic pattens in a network, the attacker will be able to identify the critical nodes. The network relies on these particular nodes 
to pass information through the network. These can either be a node that just happen to be located in a critical route or that have a more sophisticated system and/or more power. The attacker can target these critical nodes in order to more effectively bring down the network. A similar method to the homing attack is to flood the network with connections. By opening many connections to the victim, the attacker will cause the victim to consume its battery. This also will not allow this sensor to receive any legitimate connections, thus preventing it from participating with the network [41].

Misdirection and black hole attacks involve a similar method for compromising the network. Both vectors rely on the attacker to already have packet access to the network . The misdirection attack will take all the packets it receives and forward these packets in the wrong direction. This should cause the network to spend more battery power than necessary to correctly route this information. This route is usually chosen to target a single node that is likely to be a critical node [41].

A black hole attack is an advertisement of zero-cost routes. These zero-cost routes will make the sensor routing tables think the new node is the most power effective route. This has a two-fold effect: (1) the target node will be DOS attacked by its own system and (2) all of the packets can be lost to the attacking node. In the second case, the attacking node will simply discard the message or selectively forward the ones it wishes to allow. Either method will result in a compromise in the sensor network [41]. 


\section{CHAPTER III THEORY AND DESIGN}

\subsection{Wireless Sensors}

A wireless sensor is a miniature computation device capable of detecting information about the environment, processing this information, and the ability to transmit this information to other locations. Each sensor is capable of functioning independently of the others. Multiple sensors comprise a sensor grid or network. This is the functional object which is designed to perform some kind of monitoring task.

This project uses the Mica2 mote and the MTS300 sensor board. The Mica2 mote (FIGURE 3.1) is powered by two AA batteries with a lifetime upwards of one year utilizing the sleep modes. There is 128 Kbytes of available flash memory for the program space. The board supports Universal Asynchronous Receiver/Transmitter (UART) serial communications. This device has a 10-bit Analog to Digital Converter (ADC) for monitoring analog sensors. The power consumption is $8 \mathrm{~mA}$ in active mode and less than $15 \mu \mathrm{A}$ in sleep mode. This small amount of current allows this device to have a lengthy lifetime [10].

The multi-channel radio onboard the mote allows for bidirectional communication within the network. The center frequency is $916 \mathrm{MHz}$. There can be four or five channels of communications utilizing frequency-shift keying (FSK). The data rate of the communications is 38.4 Kbaud. This device typically has a 500 foot line-of-site (LOS) range outdoors. The radio draws $27 \mathrm{~mA}$ transmitting at maximum power and

$10 \mathrm{~mA}$ receiving data. The radio sleep mode draws less than $1 \mu \mathrm{A}$ [10]. 


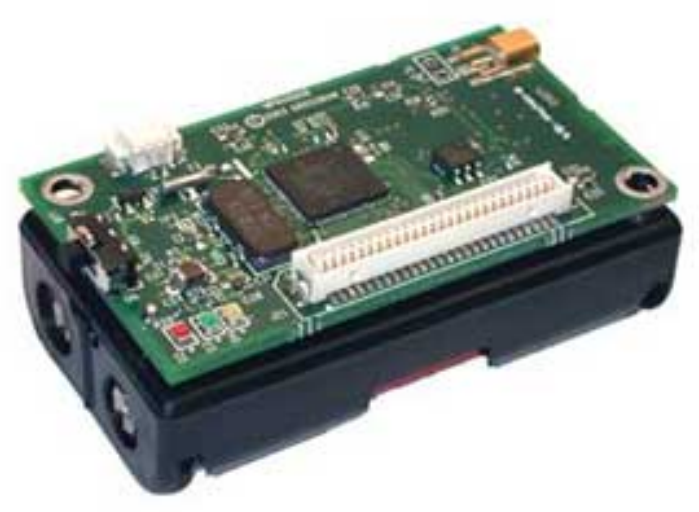

FIGURE 3.1 - Mica2 mote package from XBow [10]

The mote has 3 LEDs to provide feedback from the system. These are programmable through TinyOS and their functions are only what the developer programs. The mote is $58 \times 32 \times 7 \mathrm{~mm}$ and weighs 18 grams. An expansion connector of 51 pins is utilized to attach any number of sensor boards to the mote [10].

The MTS300 sensor board (FIGURE 3.2) is the basic sensor board which can detect light levels, sound levels, and temperatures, and is capable of sounding a tone as a feedback mechanism from the mote software. TinyOS can control how each sensor operates, such as turning the individual sensor on and off to conserver power. This board is not critical to this thesis's research; however, it will provide data to the sample simulation and tests [10].

\subsection{TinyOS Framework}

TinyOS is the open source framework which is utilized to develop and run embedded sensor networks. It is designed to support concurrency intensive operations required by sensor grids with a minimal hardware configuration. TinyOS utilizes a special language and compiler called nesC to program and develop for individual sensors [36]. 


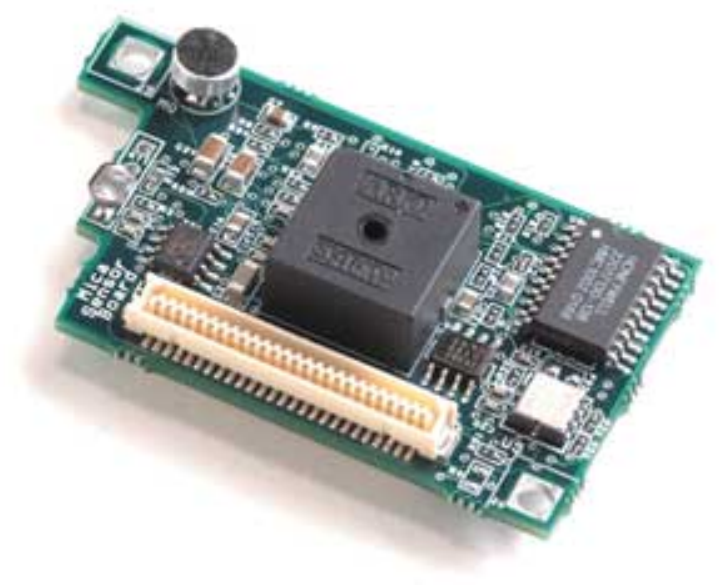

FIGURE 3.2-XBow Sensor Board with Temperator, Sound, and Light sensors [10]

The TinyOS Framework is designed to be completely modular. By wiring components together, nesC allows for this component-based design with a C-like syntax. The modules can be written to perform a number of tasks from wireless grid communication to hardware interfaces to encryption routines. The robustness of the modules allows for easy and powerful expansion of the system, within bounds of the limited hardware [36].

\subsection{TOSSIM}

TinyOS SIMulator (TOSSIM) is the simulator developed for a TinyOS environment. This simulator is useful when developing sensor network applications. The sensor's small form factor and remote locations prohibit examination of the state of the sensor. This is where TOSSIM becomes very useful. The simulator can scale to more nodes than a developer usually has available. This scalability of the simulation allows developers to determine what will happen when the network scales to a particular size. Since TOSSIM emulates the operations of the sensors to the bit level, any possible experiments can occur and should function the same on physical 
sensors. This simulator provides a drop-in replacement for the actual sensor and all of the tools utilized to communicate and test the networks can be utilized within the simulator [23].

A graphical tool, TinyViz, allows visualization and interaction of sensors with the user. This is very useful to graphically see what the network is doing. The basic plugins allow for monitoring, in a graphical fashion, the radio packets and other communications, sensor status, and anything else which can be monitored. This graphical tool allows the user to move and manipulate the sensor positions to test the network under a variety of conditions.

\subsection{Energy Consumption}

Energy consumption is a direct result of a wireless transceiver and processing of data. The goal is to minimize the energy consumption while maximizing security. There are main methods of increasing the energy efficiency of the device. The first

is to build more power efficient hardware. The other method is to develop more efficient software to take advantage of the hardware. TinyOS and some of the motes support sleep states to attempt to preserve power. The disadvantage of sleeping is that the node can not respond to anything while it is sleeping. Features and energy efficiency do not go hand in hand and sacrifices must be made as to which one is more important.

\subsection{Design of Algorithms}

This section describes how the algorithms were designed. It provides the necessary figures and discussion to replicate these algorithms. 


\subsubsection{Session Keys}

Session keys are utilized for node to node communication. They exist only between any two communicating nodes. Session keys are typically deployed in large sensor networks and when nodes are resource constrained. These nodes are assumed to be immobile and must share a master key [22]. The BROSK method assumes the master key is the sole determining factor in authenticating other nodes [22].

\subsubsection{Soft Key Distribution}

This BROSK method forms the basis on which new key distribution procedures are built. The new process combines this procedure from Lai [22] and the soft authentication ideas from Park [32] to form a new mechanism for generating and ensuring security within wireless sensor networks.

The goal is to provide smaller keys, generated on demand as the network sees fit, to secure point-to-point communications among pairs of nodes. The authentication mechanism examines the sensors to ensure the validity of the underlying system.

Each procedure (FIGURE 3.3,3.4) will run concurrently on a pair of motes, with mote A running the (setupKey) and mote B will run the (validationRequest). These procedures will run concurrently. Prior to utilizing the key generation and authentication algorithms, all communications for the key determination occur over a pre-shared master key. This key is only to be utilized during the startup procedures of the sensor network. Once the network has been established, the generated keys are the only form of encryption.

Mote A discovers which mote it wishes to setup a key with and adds this id to a list. Mote $\mathrm{A}$ then generates four random byte locations, within the operating system code, which then computes a cyclic-redundancy check (CRC) based upon the data at these four locations. Mote A sends the four byte locations in a data packet 


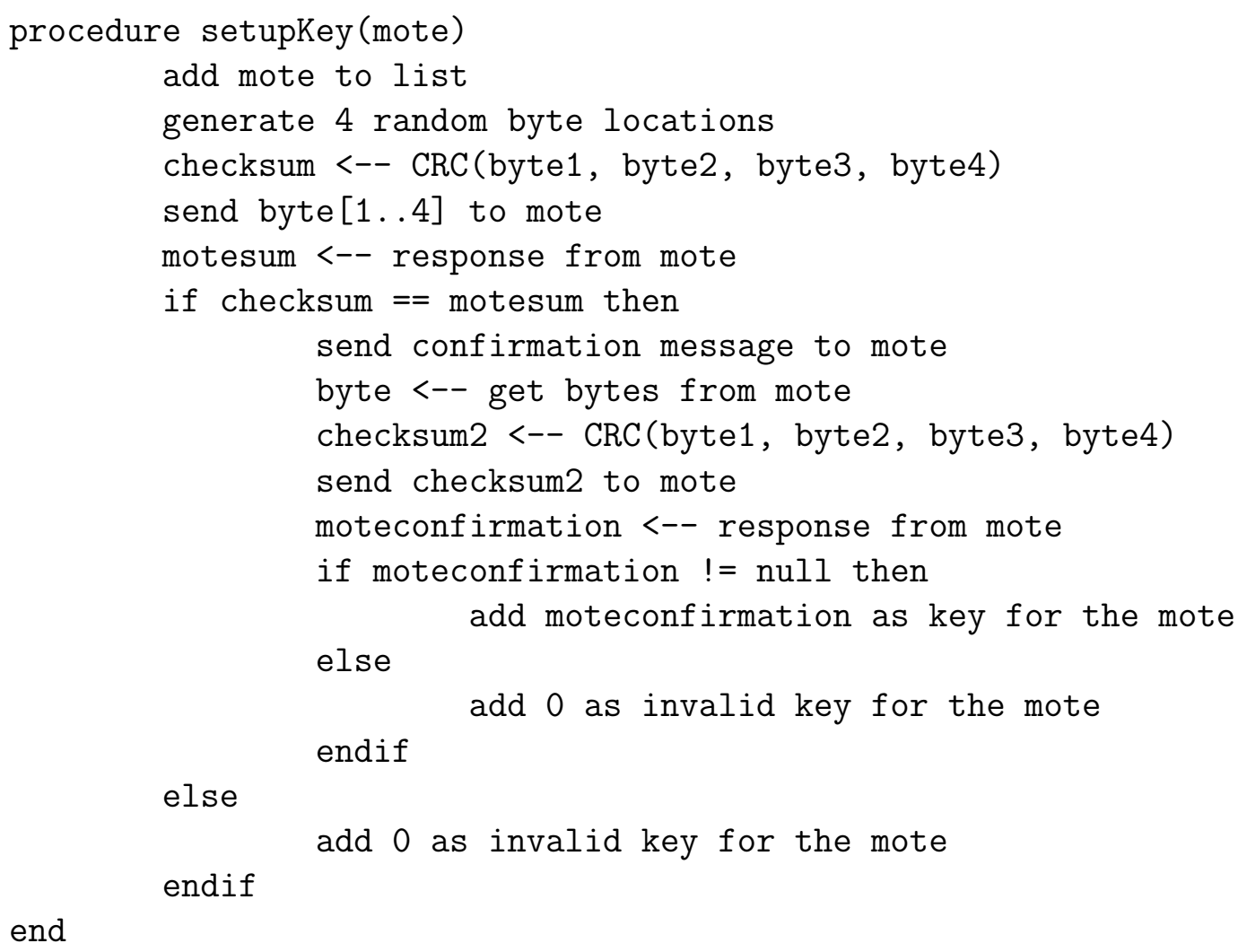

FIGURE 3.3-First half of a procedure to setup a key on a pair of nodes. 


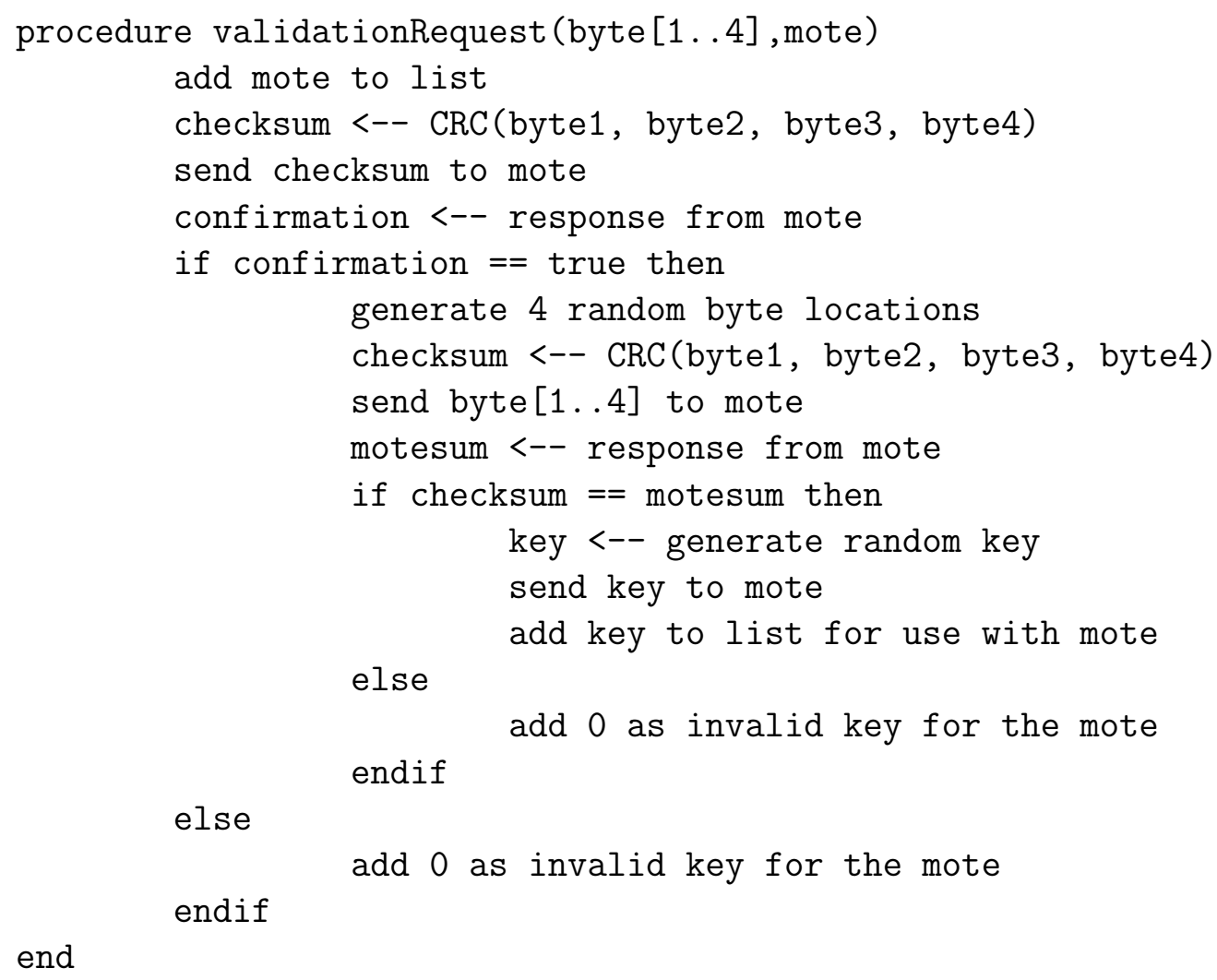

FIGURE 3.4-Other half of the procedure to setup a key on a pair of nodes. 


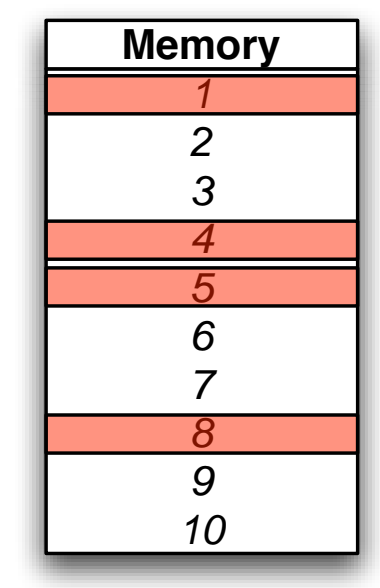

FIGURE 3.5-Example Memory Map

to mote B (Figure 3.5), the discovered mote. Mote A now waits for a response from mote B. Mote B performs the same steps as mote A and adds mote A's ID to a list. Mote $\mathrm{B}$ computes the $\mathrm{CRC}$ and returns this value inside of a data packet to mote A. Mote A check the received value from mote B to ensure it is the same as its checksum. If the checksums do not match, then mark mote B as a compromised node. If the checksums match, then send a confirmation message to mote B. Mote $\mathrm{B}$ will now generate four random byte locations and compute the CRC for these memory locations. Mote B sends these byte locations to mote A. Mote A receives four byte locations and computes the CRC based upon this information and returns the checksum to mote $\mathrm{B}$. If the checksum from mote $\mathrm{A}$ matches the one for mote $\mathrm{B}$ then mote B will generate a 16-bit cryptographic key. This key is added to the list for use with mote A and sent to mote A. Mote A will receive the key and add it to its list for use with mote B (Figure 3.6).

If anything happens in either procedure, the motes will assume a security problem has occurred and mark each other as invalid nodes. This will ensure the security and integrity of the procedures by not allowing a method for attack against the algorithm. This deny-on-error policy should guarantee these procedures to be hardened. 


\section{Sensor 1}

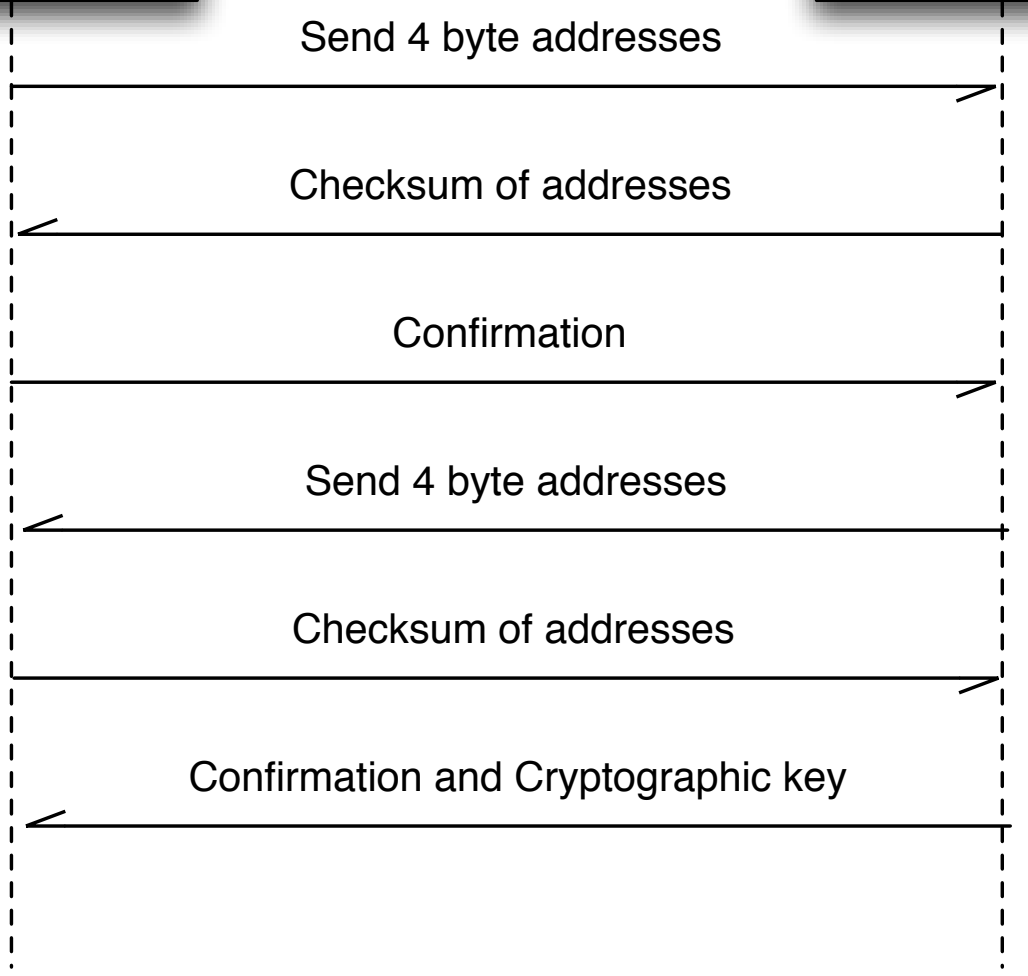

FIGURE 3.6-Algorithm Message Passing 
The controlling network layer should ensure the delivery of the data packets, even in the event of failures and interruptions. These two procedures will provide a 16-bit symmetric key for utilization between the two motes. This key is only valid for communication on this link. If a mote wishes to communicate with a different node, it must utilize the appropriate key for the link. This algorithm utilizes the 16-bit key; however, this key length may be changed to whatever is desired, within the limits of a single data packet. The current structure limits this key to be 160-bits.

Multi-hop communications will take a slight power and performance hit when routing information with the unique point-to-point keys. In order for data to route from one node to another node via multiple hops through different sensor nodes, the data packet will have to be decrypted and re-encrypted at each hop in the route. This will take only slightly more effort than a network utilizing a single master key. The major benefit to the point-to-point keys is with the extra security from node compromise. The losses from the decryption and re-encryption should not affect the power consumption and performance of the network very much. There will be a larger problem with power in multi-hop communications. 


\section{CHAPTER IV IMPLEMENTATION}

\section{1. nesC}

$\mathrm{NesC}$ is the language in which TinyOS is written. All modifications to the TinyOS system must be implemented in this language. NesC is a language for programming structured component-based applications. It has a C-like syntax, but supports the concurrency model from TinyOS. NesC allows for mechanisms for structuring, naming, and linking software components into embedded applications. This facilitates the designers in building components for complete concurrent systems [36].

One benefit of the nesC language is the ability to do extensive compile time testing and validation of software. The compilers can identify and detect many errors and problems both in syntax and in concurrency within the language. This provides valuable information to the developer. Concurrency errors are significantly reduced through the use of code checking [36].

The language, nesC, provides a component object. The application is comprised of one or more components linked together to form the application. Components provide and use interfaces. This is the only interaction available to the component. The interfaces are bidirectional for communication and interaction. The interface declares a set of methods called commands which must be implemented by the interface designer. These methods are similar to the sense of method in Java where they are part of a class. The other set of methods the interface declares is

events. Events give the component a method to force the application designer to 
provide the implementation which will likely be unique to the individual application. These events are similar to Java interfaces. All events must be implemented to utilize the component [36].

There are two different types of components in a nesC application. The first is a module. Modules provide the application code for the platform. The second is the configuration. The configuration links the interfaces of the provided models together to form a working application. This linking is known as wiring [36].

The concurrency model is a unique design that is necessary for TinyOS to function with the hardware. The system contains two type of handlers: task and hardware event. Tasks are run in such a way that they run to completion and do not preempt one another. Hardware events are similar to tasks in that they run to completion; however, they may preempt another hardware event or task. Race conditions become a problem when multiple asynchronous handlers occur and can preempt each other. This is why TinyOS contains checks for race condition. There are methods that the programmer can employ to resolve the race conditions reported by the TinyOS compiler. These are discussed in the NesC documentation [36].

\subsection{Application Code}

Data and control values must be passed between motes in order to accomplish the key establishment protocol. A TOS_Msg (FIGURE 4.1) structure is the building block on which custom structures may be added for data exchange. The message contains the destination address (addr) and type of message (type). A group can be specified if necessary and the length of the message is stored. The data structure is where a custom structure can be added for different tasks. A CRC is computed prior to transmission to ensure the integrity of the data within the packet.

For the new protocol, an IntMsg structure provides the necessary information. The source of the data (src) is transmitted with the structure. This allows the 


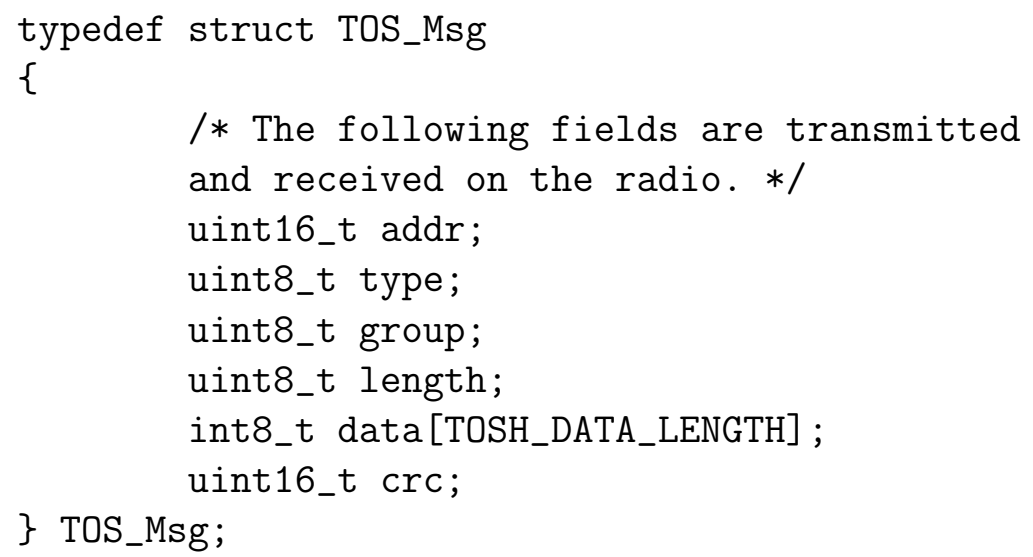

FIGURE 4.1-TOS_Msg Structure (from AM.h)

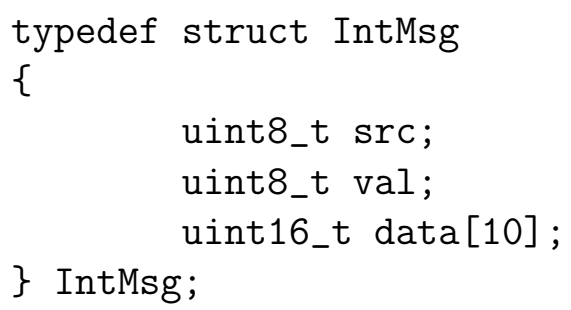

FIGURE 4.2-Int_Msg Structure

receiving mote to identify where the data is coming from and is important in the key establishment procedure (FIGURE 4.2). Message types are stored in the val attribute. This is utilized for tracking which data is contained within the data portion of the structure and is how the setup key algorithm keeps everything in order.

Passing of message between motes prompts the inclusion of the "val" attribute into the IntMsg structure. This is required to keep track of the process of keygeneration. Each step of the key-generation algorithm updates this value with where its message should be routed. The receive method, which processing incoming packets, examines this information and calls the appropriate function to process either the next stage of key establishment or any other user defined procedure. This message handler 
is responsible for the decryption of the incoming message and routing this message to the appropriate location.

A procedure named sendSecure takes the place of the usual Sendmsg.send method. This function provides the required encryption procedure to encode the data portion of the TOS_Msg structure. If a point-to-point link is not setup for the particular communications, then this will initiate the setup procedure before sending the current message. The current message is stored in a backup object and completed at the conclusion of the key setup procedure. Since multiple messages are being passed between motes, a flag is set to indicate the presence of the backup data and the last thing the mote does is check this flag before completing the key establishment procedure.

The encryption procedure is a simple exclusive-or (XOR) between the data bytes and the encryption key. This is the simplest form of encryption possible as well as the most power efficient. Initially, the encryption occurs with the master key built into the system; however, this is only utilized for the initial creation of a point-topoint key. Once the point-to-point key is established, all other communications occur with the generated key. The lifetime of a key can be set so the system will renew a particular key after this limit is reached. This will allow for key changing to prevent unauthorized access to the data streams. Each mote contains a list of keys for use with its communications that are stored in RAM. A physical attack on the mote will not easily allow the contents of this RAM to be determined. 


\section{CHAPTER V TESTING AND RESULTS}

\subsection{Testing Overview}

There are two main components to the system: key generation and network communication. The first test is for the key generation and establishment of dynamic point-to-point keys; while, the second test is to ensure the scenario is functional for multi-hop communications. Final testing combines both the prior tests to form the basic structure of the sensor network security. This is tested for correctness.

\subsubsection{Testing Key Generation}

Testing the key generation algorithm involves setting up two sensor nodes (FIGURE 5.1) and having them establish a shared key. Through the use of debug messages, TOSSIM can be utilized to test the key generation algorithm. Appropriate messages are placed at critical sections of the algorithm to display the contents of memory concerning the key generation. These messages are also used to show the progression of the algorithm, much like hand-tracing an algorithm.

For testing purposes, the key generation algorithm is slightly modified. Instead of reading data directly from the program code, byte data will be read from the data EEPROM. The structure of TinyOS places a limitation on reading and writing information from within the program EEPROM. This change will only affect the implementation of the algorithm, but will provide similar features and energy consumptions. For a practical implementation, TinyOS will need to be modified to allow 


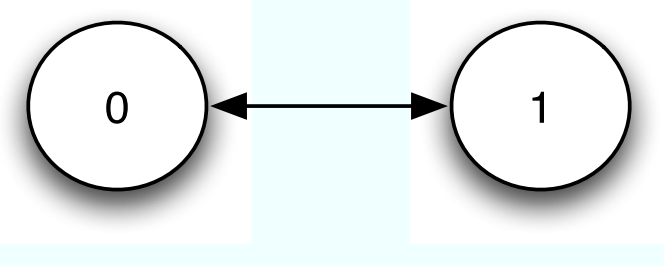

FIGURE 5.1-Key Generation Test Setup

reading of the program EEPROM data.

Node 0 and 1 will utilize the master key to secure the initial communication and establish a shared secret key. Once this key is established, some data will be transmitted between the nodes to ensure the communications and encryption are properly implemented. A default key utilization length of five transmissions is implemented within this application.

The following is the output excerpts from a sample run of the test. These results (TABLE 5.1) show the progression of the key generation algorithm. The debug dump message show the state of the data contained within the packets as well as the node transmitting and a status value for process control. Each step of the algorithm will utilize a different number of elements within the data packet. At the end of the process, an encryption key is generated and provided to each node for their private communications.

\subsubsection{Testing Network Communication}

Testing the network communications involves establishing a sensor network, in simulation, to verify the communications are functioning. This test will pass information from the initial node through a series of hops to the final destination node. (FIGURE 5.2) This is done with no encryption of any kind on the network, just a basic network. 
1: TIMER Message: 0

1: Dump: 17 12321,46472,23066,38187,6976,6047,3617,15709,23461,38485

1: Dump2: 17 12321,46472,23066,38187,6976,6047,3617,15709,23461,38485

1: Dump: 1100 7612,6759,5585,2749,0,0,0,0,0,0

0: Dump: $010161560,0,0,0,0,0,0,0,0,0$

1: Completed sending message

1: Dump: 1102 11653,10846,9704,14980,12345,12345,12345,12345,12345,12345

0: Completed sending message

0: Dump: 0103 53954,42243,19085,34196,12345,12345,12345,12345,12345,12345

1: Completed sending message

1: Dump: 1104 43365,6759,5585,2749,0,0,0,0,0,0

0: Completed sending message

0: Dump: 0105 2000,38202,31412,46509,0,0,0,0,0,0

1: Completed sending message

1: Mote Link $1<-->0$ is complete. Key: 2000

1: Dump: 17 12321,46472,23066,38187,6976,6047,3617,15709,23461,38485

0: Completed sending message

TABLE 5.1

Simulation Results for Key Generation Testing 
4: Sent Message: (Node,value) 3,1
3: Received Message: (Node,value) 4,1
3: Sent Message: (Node,value) 2,1
2: Received Message: (Node,value) 3,1
2: Sent Message: (Node,value) 1,1
1: Received Message: (Node,value) 2,1
1: Sent Message: (Node,value) 0,1
0: Received Message: (Node,value) 1,1

TABLE 5.2

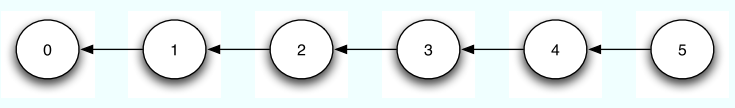

FIGURE 5.2- Network Communications Test Setup

This test will send data from node 5 to node 0 by passing the data packet to each node in the route. Debug messages are produced by each node upon reception and transmission of a data packet. This allows for easy analysis of the route and the functionality of the sensor network. The results (FIGURE 5.2) show the debug messages and progression (FIGURE 5.3) of data through the network. The arrows are the messages passing to the next node in the network. Meanwhile, the circle is a broadcast message from node 0 looking to see if any other nodes are in the network. The debug messages show node 4 sending a message to node 3 , then node 3 sends a message to node 2 and this continues until node 0 receives the message. This is just an excerpt from the simulation data; the rest can be found in appendix II. 


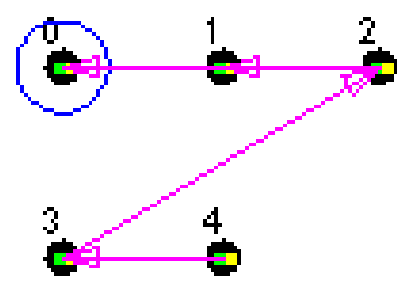

FIGURE 5.3-TOSSIM Network Test

\subsection{Experimental Design}

Given the procedures work as designed, the question is how much energy is it going to cost to deploy this algorithm and security system? This cost affects the capabilities of the network; however, when security is required, what kind of threat is there to the integrity of the communications and networks.

\subsubsection{Sample Tree Application}

The following application is for the testing of a basic multi-route wireless sensor network. It is developed and simulated completely on the computer. The goal is to monitor information at the bottom of the tree and pass this information through the sensor network back to the root node. This scenario is used because of the ease of implementation and predictability of the network.

Nodes follow two simple rules. First, generate a random number between zero and seven and send this information to the parent. This is the data the network is to provide to the root node. This routing of information ensures delivery to the 


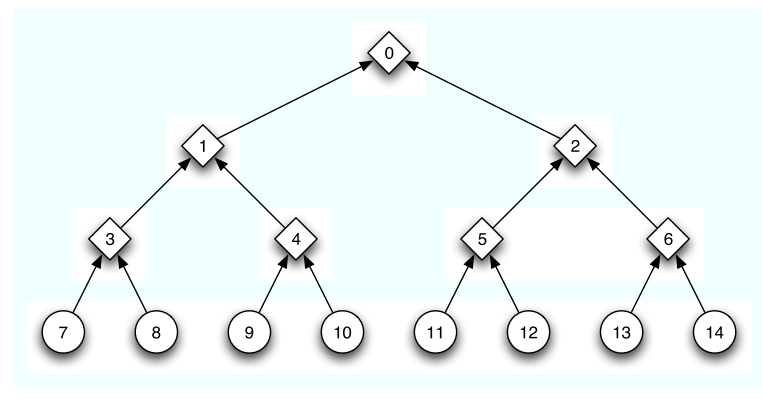

FIGURE 5.4-Routing diagram of the Tree test application

root node. With this first rule, the nodes will just generate numbers and send their information to their parents. This does not provide a route or method for multi-hop transfer. The second rule is if a node receives a radio packet with data, the node will cease to generate data and become a intermediary node within the tree structure. Once a node has determined if it is an intermediary node, its function changes to receive the data from both of its children and determine the larger of the two. This information is then passed along to its parent. The circles denote data/sensing nodes. This is where the data is generated to be passed along to the root node. The diamonds denote intermediary nodes whose only goal is to pass the largest of its children up the tree. The directed graph shows the routing of information to the root node. (FIGURE 5.4)

This application is tested with TinyViz for two scenarios. The first one being a basic unencrypted network of 15 nodes. TinyViz, with the directed graph and radio link plugins enabled, shows the radio packets in the network and is utilized to test and verify the simulation. Power profiling is tested with the test scripts (APPENDIX I). The profiling simulation will run for 180 seconds. Node power consumption is reported in Figure 5.5.

The second test is with the new encrypted point-to-point communications. The parameters are kept the same. 15 nodes will be utilized for the test and the power profiling will run for 180 seconds. Node power consumption is reported in 


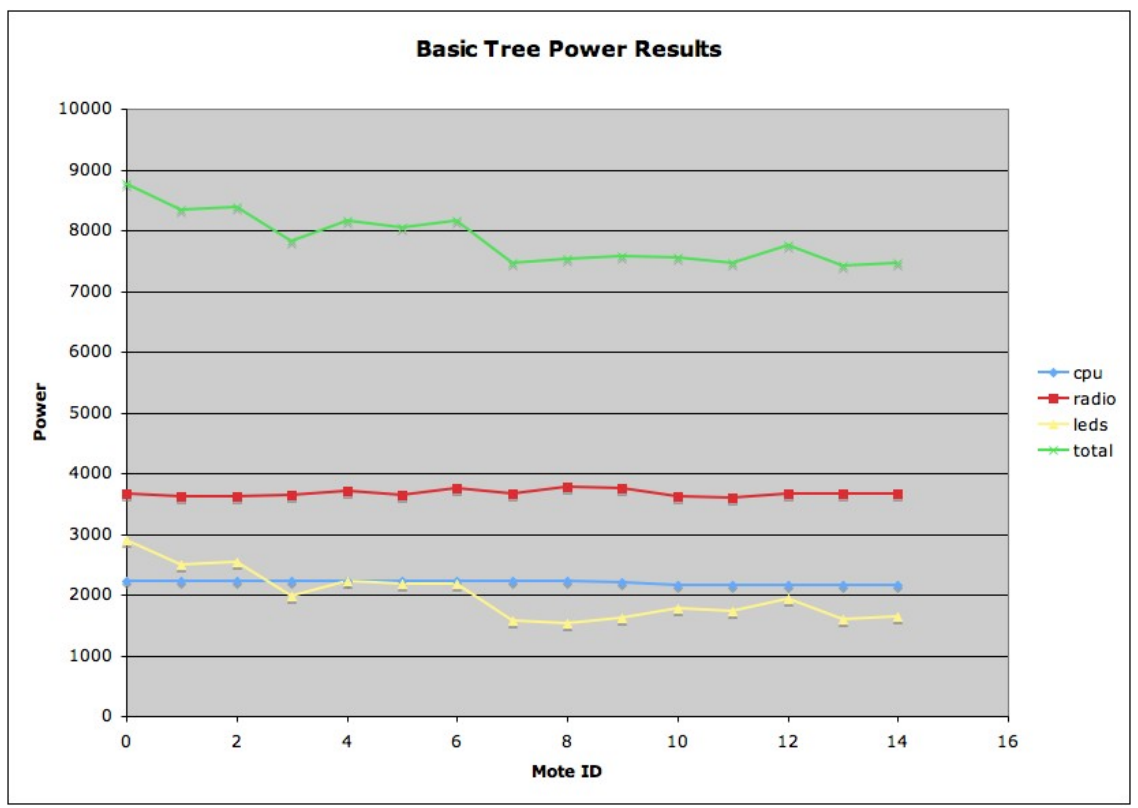

FIGURE 5.5-Power consumptions for the Basic Tree Applications

Figure 5.6.

The differences between each test are provided in Figure 5.7. The power capacity of a typical AA battery is 2700 mah. The motes utilize two batteries, providing them with 5400mah of power. The average utilization of energy (mJ) over the three minute trial is $7836 \mathrm{~mJ}$. This is equilivent to $\frac{7836 \mathrm{~mJ}}{180 \mathrm{sec}}=43.5 \mathrm{~mW}$. $\frac{43.5 \mathrm{~mW}}{3 \mathrm{~V}}=14.5 \mathrm{ma}$ is the amount of current consumed each second by each mote. The approximate lifetime of a sensor running at full power with no sleep is $\frac{5400 \mathrm{mah}}{14.5 \mathrm{ma}}=372$ hours $=15.5$ days . This is independent of the encryption technology overlaid on the communications.

An initial comparison of the results shows no noticeable differences between the two tests. Since the tests ran for the same length of time this is expected. With each mote running full speed, the cpu consumed a similar amount of power across both tests for all motes. The same holds true for the radio power consumption. The LEDs caused some variance to the total power levels. This is due to asynchronous nature of a mote. There is no guarantee exactly when the led will change.

These two tests show the energy consumption of an active running sensor 


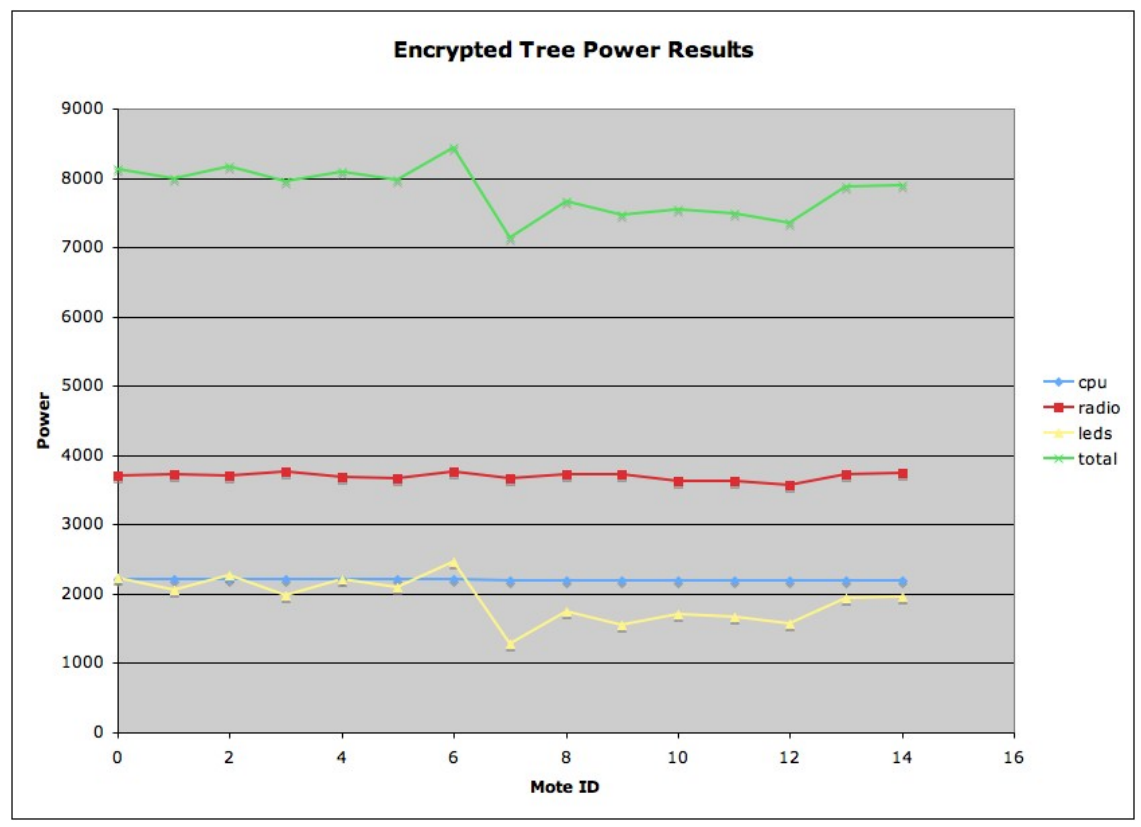

FIGURE 5.6- Power consumptions for the Encrypted Tree Applications

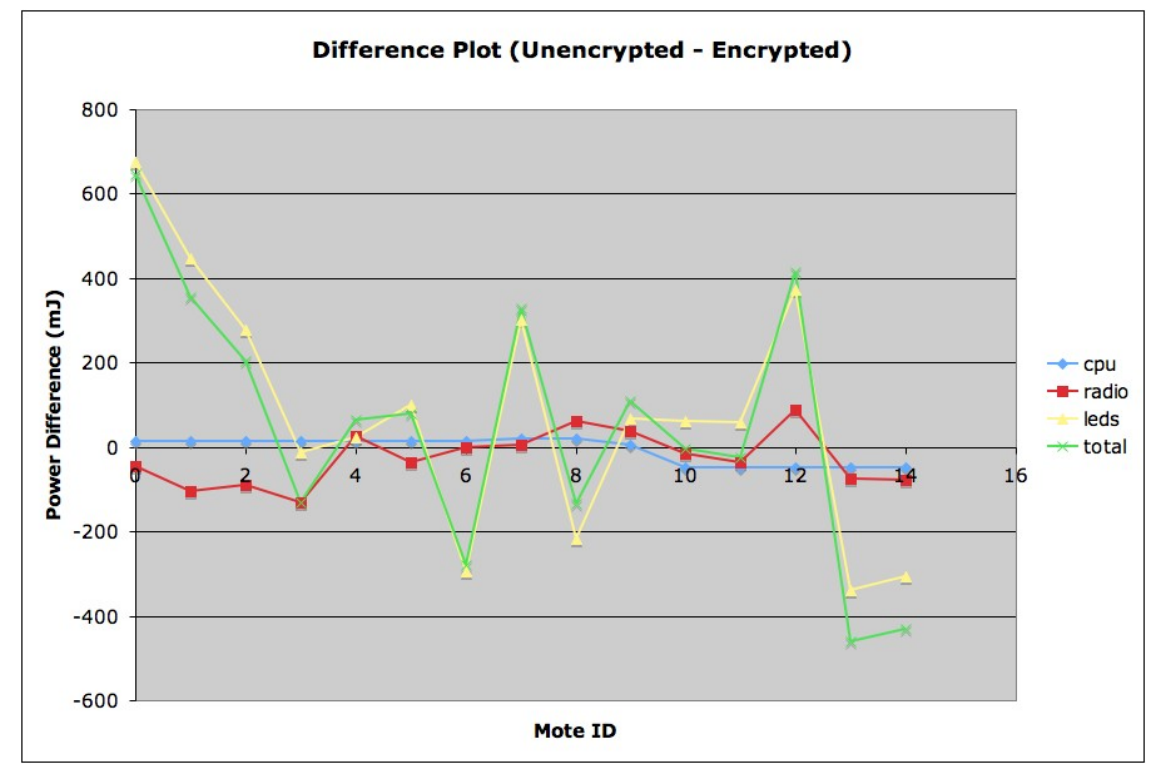

FIGURE 5.7-Power consumptions difference between encryption and plain 


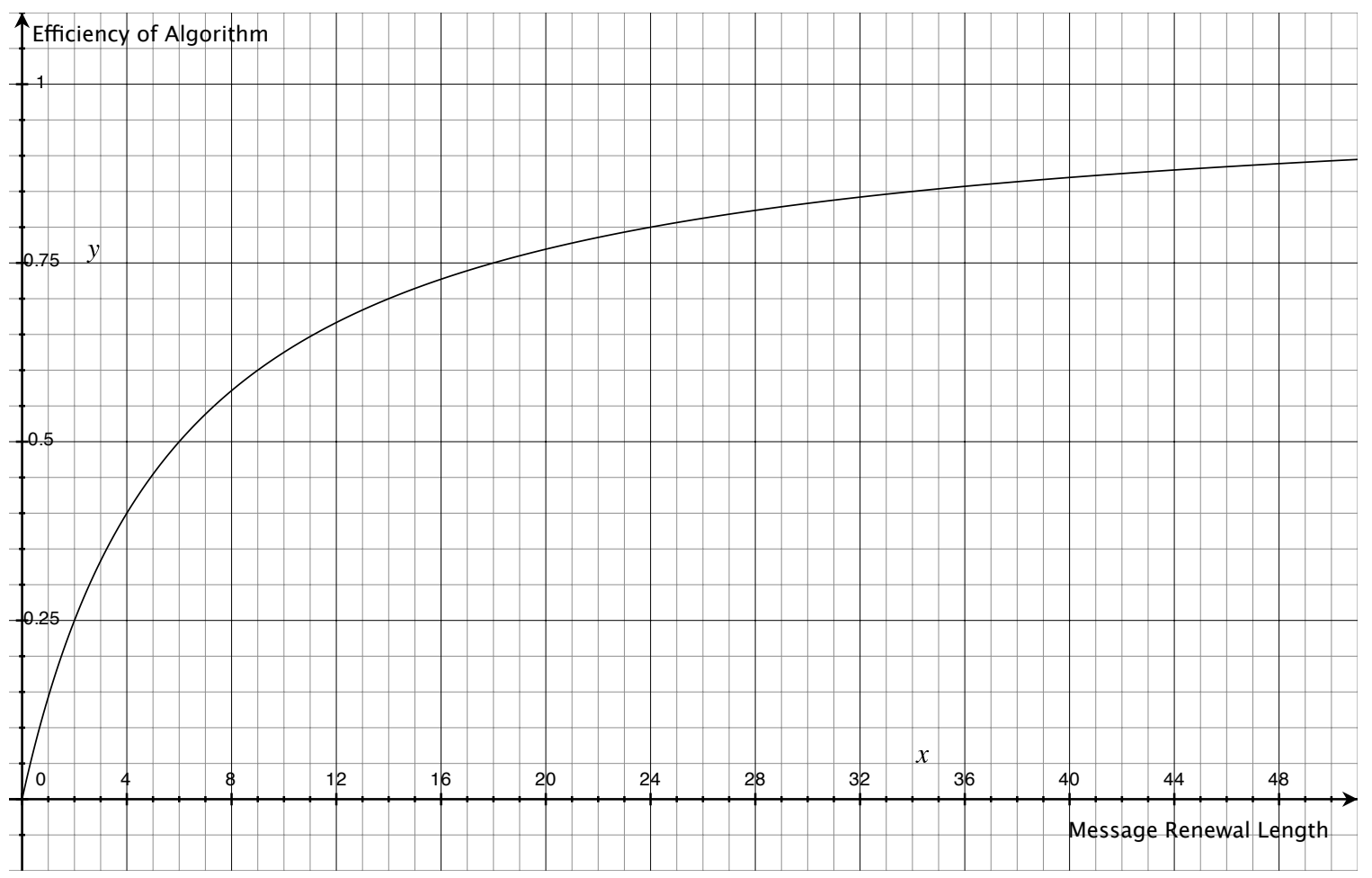

FIGURE 5.8- Power Efficiency of the Algorithm

network. Due to the overhead within the protocol (radio messages and cpu time), the encrypted test did not complete nearly as many bottom to top communications as the basic unencrypted test. For each effective message, the encrypted technique requires on average two radio message to send. There is an overhead of radio packets in the key creation and renewal algorithms. This overhead is the component to minimize for optimal power consumption.

The actual efficiency of the new protocol is 45 percent. This is from the total renewal period of messages. 11 total messages for sending 5 data messages yields the 45 percent efficiency. Figure 5.8 shows the effects of changing the renewal time (number of messages) before requiring a new key to be generated. A 45 percent efficiency show that the encrypted version of the software will only provide 45 percent of the amount of data in the given time period and energy consumption level. 


\subsection{Security}

There are three main security problems with the new protocol and with any sensor network. Intrusion into the network is a serious problem and can result in the collapse of the functional network. The integrity of the wireless encryption is critical to keeping intrusions at bay and the communicated information secure. Wireless integrity is the most likely attack method and potentially the most damaging. The last major topic is that of misuse and abuse of the sensor network systems. Once an intrusion has occurred, the attacker will be able to manipulate the network at will and change data within the network.

\subsubsection{Intrusion}

Intrusion into the sensor network involves breaking either the encryption keys or compromising the nodes. A compromised node is a significant problem for the safety of the network. This compromised node can also be used to affect the network communications through the use of flooding or black-hole attacks. These attacks mostly depend on how the routing is implemented within the wireless network. Many of the problems occur when the compromised node's security keys are discovered. The new key establishment protocol protects this network communication because each node-to-node link is unique. If a link is broken, only the compromised encryption is lost, not the rest of the network. If this link is discovered, through intrusion detection means, the area around the compromised key can be isolated and removed from the active network. The compromised key allows incorrect and misleading information into the network and the attack will be able to create an environment that does not exist. For example, a sensor network is monitoring an area for vehicle movement. A compromised node(s) will be able to simulate this motion through data injection into the network. Another method is to utilize the nodes to show normal data in the 
presence of vehicle movement, preventing the network from detecting it.

The new key establishment protocol provides an alternative method to the problems associated with pre-distribution procedures. These key pre-distribution methods have significant problems when a key compromise occurs. Attackers usually will acquire access to all the network's communications once the key or node has been compromised. All of the algorithms for encryption and decryption will be visible from this compromised node. These procedures will provide insight into how the encryption layer is constructed on the network and allow for a targeted attack against the network's security. For example, a compromised node has allowed the attacker to discover the encryption key for the network. Most likely, this node will contain enough information to easily communicate with the other nodes. This communication is all that is necessary to allow misinformation to be injected into the network.

\subsubsection{Breaking the Encryption}

The most likely attack on a sensor network is a compromise of the wireless encryption. As discussed in the prior section, this can be accomplished by discovering a sensor node and examining its code to determine how the encryption works and the relevant keys. Another method is to attack the wireless signals directly. This is either a passive or active attack. In the passive attack, the wireless traffic is collected from the sensor nodes. This traffic is analyzed on a powerful microcomputer in an attempt to discover the data within the communications and the key utilized in the encryption. An active attack involved sending and receiving data packets at a sensor node in an attempt to discover how the encryption works. This attack is usually detected quickly and stopped before any significant damage can occur.

For example, compromising a 16-bit key with a powerful microcomputer, in

the worst case, one needs to examine $2^{16}=65536$ different keys and $\frac{2^{16}}{2}=32768$ different keys, for the average case. All of these keys would have to be tried against 


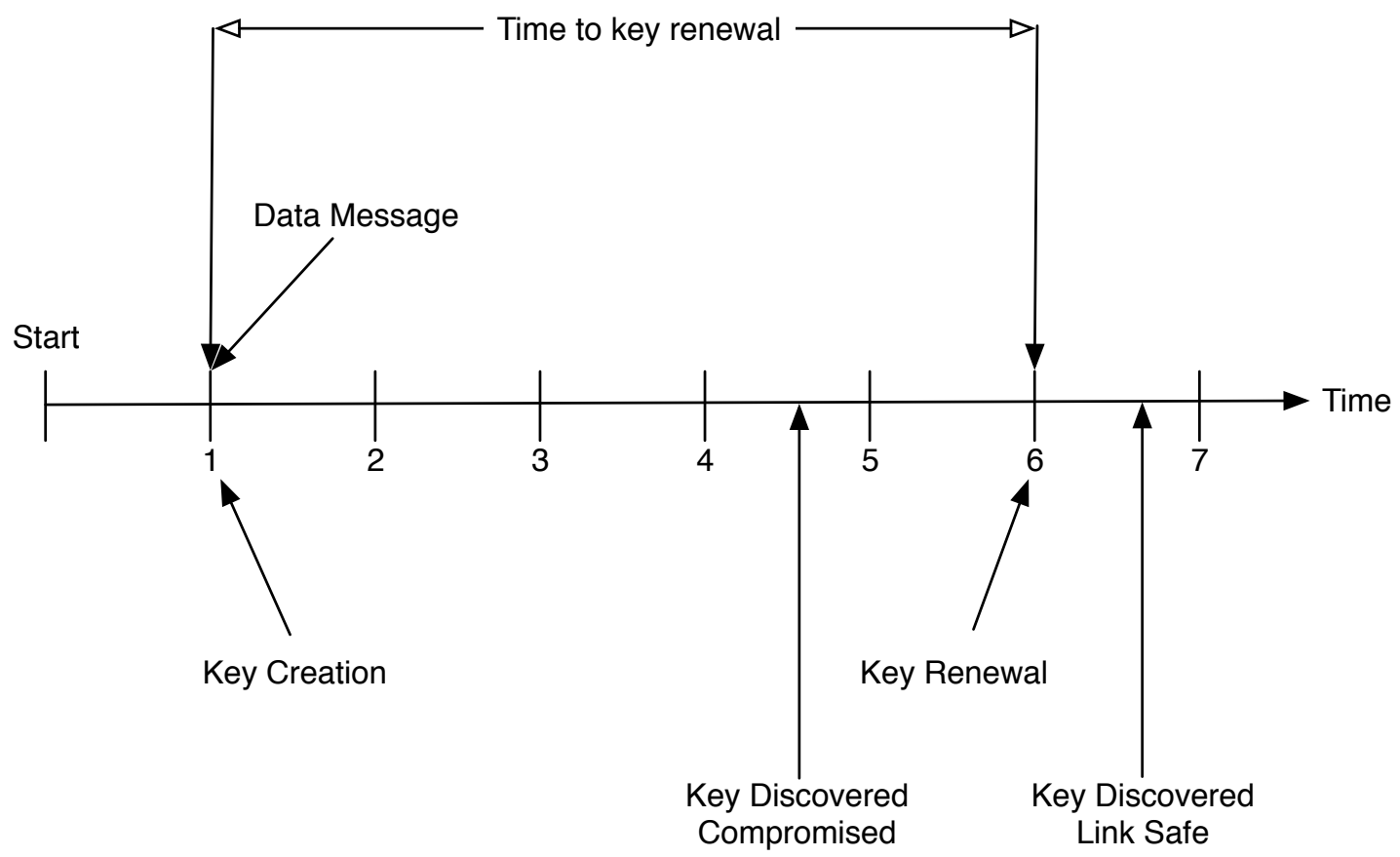

FIGURE 5.9-Key Renewal Algorithm

the captured data. Given, the encrypted data portion of the example structure is 20-bytes long and the keys are 16-bits (2-bytes) in length, the computer would have to perform $2^{16}$ keys $\times \frac{20 b y t e s}{2 b y t e s} \times 5$ packets $=3,276,800$ trials. Assuming the computer can perform each trial in 1ms, this computation will take 3276.8 seconds which is 54.6 minutes to try all the keys. This brute force method is practical if the key renewal time is longer than it takes to try the keys and make a decision about the correctness of each key. This is the minimum time required to break this encryption. Many other factors influence the strength of encryption. A longer key will require more time to evaluate the possible keys. For each bit that the key grows, the time required to check all the keys doubles. This example assumes the decryption routines are known. A more sophisticated encryption technique, such as a Advanced Encryption Standard (AES), would better secure the data. The price of security in sensor networks is limited lifetime and speed. AES will consume a lot more power than XOR (Figure $5.9)$. 
The difficult part of breaking any type of encryption is determining if this trial contains the actual key. The data can be arranged such that there are 65536 different sets of attempted decryptions, with each set containing 5 separate packets. One method for attempting to discover the key is to look at the decrypted data (spanning all recorded data packets) for decryptions showing "reasonable" values. These reasonable values will have to be determined from environmental measurements of the data being sensed and detailed knowledge of the outputs of the sensors on the devices. If the device's sensor is a custom design and not widely available, it may be necessary to capture a device and experiment on the sensor in order to determine "reasonable." Data packets contain little information (20-bytes max). The process of breaking the encryption is going to be difficult given this small sample size. The ability of the attacker to determine the type of information contained within this data packet is critical to the speed and certainty with which the encryption may be broken.

Longer key lengths or more sophisticated encryption techniques will better secure the data and communications; however, the cost to implement and deploy these techniques may be more than the sensor networks can handle. Consider the prior example, except utilize a 32-bit key instead of 16-bit key. This means there are $2^{32}=4,294,967,296=4.3$ billion keys available. If the same computer is utilized to attempt to break the encryption, the computation will take 107,374,182, 400 trials which results in about 3.4 years of computation. There are $2^{160}=1.461 \cdot 10^{45}$ keys possible in a 160-bit key length scheme. The processing time for brute-forceing this length of key is $4.63 \cdot 10^{37}$ years. This is the maximum key length supported by the data structures. There is a significant cost to compute cryptographic keys and encrypting the data on the sensor nodes prior to transmission. A similar cost occurs when the packet is received and decrypted. For this reason, small key sizes should be utilized for the encryption. The cost of key generation and encryption outweighs the 
cost of renewing a smaller key more times in a given period.

\subsubsection{Misuse or Abuse}

Misuse or abuse can occur when a foreign entity gains access to the sensor network. This is usually done through intrusion by a physical means or breaking the wireless encryption of a network. Once access is gained, under typical pre-distribution schemes, the intruder should be able to identify the encryption keys to most of the other network nodes. This will allow the exploitation of these nodes through the use of target messages or different routing of packets to misinform the intended node. This misinformed node will act as if the messages it received are valid and perform the necessary actions based upon the programming and data. Misinformation will allow the attacker to hide real information from the network, causing blind spots in the sensing ability of the network. The sensor can be fooled into thinking they are passing information along about a target, when in reality, there is nothing happening at that location.

This new point-to-point protocol separates each node from any other node more that a single hop away. This isolates the nodes into groups based on their communications. When a particular node has been compromised, only the data associated with the node needs to be invalidated. Compromised nodes do not provide keys for the rest of the network. The advantage of using the point-to-point protocol verses some kind of pre-distribution key is the integrity of the other point-to-point links is preserved. If the infrastructure supports detection of malicious nodes, this method will allow the entire rest of the system to continue to function unimpeded.

Abuse occurs when malicious nodes or outsiders attempt to consume resources within the sensor network. For a malicious node case, the protocol can do nothing to prevent DOS attacks on the rest of the network. The only thing the sensors can do is to ignore, as best they can, the incoming data packets. This does not stop the 
attack but hopefully reduces the impact on the sensor network's lifetime. The abuse can occur as a flood of data or attempts to change and disrupt the routing of the sensor network. There is no protection given by any encryption protocol to this type of abuse and the point-to-point protocol does nothing to stop this. The only hope is to prevent the abusive node from entering the network with the use of the encryption and not rely on stopping them once inside. 


\section{CHAPTER VI CONCLUSIONS AND FUTURE DIRECTIONS}

\subsection{Conclusions}

Wireless sensors will become more commonplace in the future, as the price of each individual sensor drops. These sensor networks will start to become part of everyday life and the security of their communication will become a concern. Good security is achieved through the practice of good implementation techniques and hardware technology. It is certain that security of sensor networks will be required and good security will rely on the development of new technologies.

In this thesis, a unique adaptation of two pre-existing ideas was utilized to provide a method to verify which node the communications are occurring with and develop a shared symmetric key between the two nodes. This algorithm allows individual nodes to choose who they wish to communicate with and generate a shared secret key with the node. This secret is only shared between these two nodes and is useless to any other pair of nodes on the sensor network. This secret key can be changes at necessary. The changing of the key is necessary because of the small size of the key.

Once deployed, sensor networks are at the mercy of their environment. This lack of physical security, at the sensor level, places a burden on the hardware and software. Not only is the software required to be energy efficient, it must be secure in its implementation. The hardware must be designed to prohibit discovery of the underlying software in the event of a captured node. 
A current problem with sensor networks is with the effect of a compromised or malicious node. The compromised node can do serious damage to an existing network. For a pre-shared key scenario, the attacker can use the information contained within the node to target and manipulate all the other nodes in the network. In the new point-to-point algorithm, the compromised node is only able to communicate directly with its neighbors, thus eliminating the attacker's ability to capture a single node and attack the entire network. This security style effectively isolates each node from the rest of the network in the event of a failure.

The ability of the point-to-point system to accommodate a variety of encryption techniques is a good benefit. The system is designed to support small keys to provide energy efficiency as well as large keys and sophisticated encryption algorithms to provide more security. This ability to easily allow different key sizes allows for easy expandability of the type and strength of the encryption.

The results of the energy comparison between the two different setups show the effects of encryption. The encryption is shown to consume more energy than the unencrypted version. This is expected due to the computation and packet overhead needed to setup and maintain this encryption. The single largest benefit obtained from utilizing this point-to-point protocol is the ability to change keys and the utilization of smaller key lengths.

For an actual implementation and deployment, the base system, TinyOS, must be modified to allow the application to be able to read the program code data in order to perform the validation of the sensor. For testing purposes, this was not done and data was read from a different data memory. This is acceptable for simulations; however, it should not be used for practical implementation.

The random number generator in the TinyOS package is a linear feedback shift register (LFSR). This is a basic algorithm used to generate a string of pseudorandom numbers. A significant problem for the sensors is how this LFSR is initialized. 
Currently, the seed value, is set to the mote ID. This lack of environmental seed significantly limits the random number sequences available to the mote.

This research has shown that there is a relationship between security and power efficiency. One cannot have both. There must be a line drawn between the two based upon the needs of the application. Security research is a tricky topic because it has no clear methodology for testing the security of the system. This is clearly evident in the Internet with the proliferation of thousands of viruses. Each virus takes advantage of a breakdown of security and there is no single solution to the problem. There are best practices, but no single solution. The best method for research is to examine security as a component of a larger scenario. This will allow security to be developed as part of a system, and avoid the problems of developing a security system and then finding an application for it.

\subsection{Future Directions}

Sensor networks are still in their infancy. There is still much to learn in terms of their capabilities and the drawbacks. The sensor networks utilized in this research are homogeneous. This allowed for each node to easily verify the validity of the others. A new direction for research would be to include multiple hardware configurations into the network. This will allow for larger hardware to provide the long-range communications and more powerful computation abilities. This would solve the problem with communication power consumption. The new device could contain more powerful batteries to provide the necessary resources to the nearby nodes. This approach is typically described as a flock and shepherd. This is one method of clustering nodes together. The drawbacks are in the validation algorithm. The new, more powerful nodes, will have to be able to communicate and validate the smaller sensor nodes as well as perform their own validations between other new nodes. This tiered approach to sensor networks adds complexity to the system; however, the 
benefits can be significant.

Another direction would be to improve on the random number generator. The simple LFSR would benefit from a more powerful algorithm as well as a hardware based random number generator. If a chip could be built to generate better random numbers, the whole system would benefit from the availability of these new random numbers.

The energy consumption is a good direction to pursue with new research. This is a critical component to the lifetime of a sensor network. Barring better power sources, energy consumption minimization is the only way to extend the life of a network. From this research, more sophisticated test cases are needed to better understand the energy consumption of real world networks. The example chosen is a simple, easy to test and trace algorithm. The real world applications will not be so trivial. Energy mapping of a network could show where the point of failures will occur. This mapping could be utilize to reposition sensors to better utilized the available resources. It could also be used to redefine the routing system within the network to spread out the power consumption. 


\section{REFERENCES}

[1] I.F. Akyildiz, W. Su, T. Sankarasubramaniam, and E. Cayirci. Wireless sensor networks: A survey. Computer Networks, 38, 2002.

[2] Yair Amir, Cristina Nita-Rotaru, Jonathan Stanton, and Gene Tsudik. Secure spread: An integrated architecture for secure group communication. IEEE Transactions on Dependable and Secure Computing, 2(3):248-261, Jul-Sep 2005.

[3] Michael Brownfield, Yatharth Gupta, and Nathaniel Davis IV. Wireless sensor network denial of sleep attack. Proceedings of the IEEE Workshop on Information Assurance and Security, pages 356-364, 2005.

[4] Seyit A. Camtepe and Bülent Yener. Key distribution mechanisms for wireless sensor networks: A survey. IEEE Transactions on Mobile Computing, 4(3), 2005.

[5] Srdjan Capkun, Levente Buttyán, and Jean-Pierre Hubaux. Self-organized public-key management for mobile ad hoc networks. IEEE Transaction on Mobile Computing, 2(1):52-64, Jan-Mar 2003.

[6] H. Chan and A. Perrig. Pike: Peer intermediaries for key establishment in sensor networks. Proceedings of IEEE Infocom, 2005.

[7] H Chan, A Perrig, and D Song. Random key predistribution schemes for sensor networks. Security and Privacy, 2003. Proceedings. 2003 Symposium on, pages 197-213, 2003. 
[8] Haowen Chan, Virgil D. Gilgor, Adrian Perrig, and Gautam Muralidharan. On the distribution and revocation of cryptographic keys in sensor networks. IEEE Transactions on Dependable and Secure Computing, 2(3):233-247, Jul-Sep 2005.

[9] Haowen Chan and Adrian Perrig. Security and privacy in sensor networks. IEEE Security, October 2003.

[10] Inc. Crossbow Technology. http://www.xbow.com/.

[11] Jing Deng, Richard Han, and Shivakant Mishra. Enhancing base station security in wireless sensor networks. April 2003.

[12] R Di Pietro, LV Mancini, and A Mei. Random key-assignment for secure wireless sensor networks. Proceedings of the 1st ACM workshop on Security of ad hoc and sensor networks, pages 62-71, 2003.

[13] W Du, J Deng, YS Han, PK Varshney, J Katz, and A Khalili. A pairwise key predistribution scheme for wireless sensor networks. ACM Transactions on Information and System Security (TISSEC), 8(2):228-258, 2005.

[14] Wenliang Du, Jing Deng, Yunghsiang S. Han, Shigang Chen, and Pramod K. Varshney. A key management scheme for wireless sensor networks using deployment knowledge. IEEE Conference on Computer Communications, 2004.

[15] L Eschenauer and VD Gligor. A key-management scheme for distributed sensor networks. pages -

[16] Huirong Fu, Satoshi Kawamura, Ming Zhang, and Liren. Replication attack on random key pre-distribution schemes for wireless sensor networks. Proceedings of the IEEE Workshop on Information Assurance and Security, pages 134-141, 2005. 
[17] Gunnar Gaubatz, Jens-Peter Kaps, and Berk Sunar. Public key cryptography in sensor networks - revisited. First European Workshop on Security in Ad-Hoc and Sensor Networks, August 2004.

[18] R. Gennaro, A. Lysyanskaya, T. Malkin, S. Micali, and T. Rabin. Algorithmic tamper-proof (atp) security: Theoretical foundations for security against hardware tampering. 2004.

[19] Fei Hu, Jim Ziobro, Jason Tillett, and Neeraj K. Sharma. Secure Wireless Sensor Networks: Problems and Solutions.

[20] Linqxuan Hu and David Evans. Secure aggregation for wireless networks. IEEE Workshop on Security Assurance in Ad Hoc Networks, 2003.

[21] Dijiang Huang, Manish Mehta, Deep Medhi, and Lein Harn. Location-aware key management scheme for wireless sensor networks. ACM Workshop on Security of Ad Hoc and Sensor Networks, pages 29-42, October 2004.

[22] Bocheng Lai, Sungha Kim, and Ingrid Verbuwhede. Scalable session key construction protocol for wireless sensor networks. IEEE Workshop on Large Scale Real-Time and Embedded Systems, December 2002.

[23] Philip Levis, Nelson Lee, Matt Welsh, and David Culler. Tossim: Accurate and scalable simulation of entire tinyos applications. ACM Conference on Embedded Networked Sensor Systems, pages 126-137, November 2003.

[24] Zang Li, Wade Trappe, Yanyong Zhang, and Badri Nath. Robust statistical methods for securing wireless localization in sensor networks. Proceedings of the International Conference on Information Processing in Sensor Networks, pages 91-98, April 2005. 
[25] D Liu and P Ning. Location-based pairwise key establishments for static sensor networks. Proceedings of the 1st ACM workshop on Security of ad hoc and sensor networks, pages 72-82, 2003.

[26] Donggang Liu and Peng Ning. Establishing pairwise keys in distributed sensor networks. ACM Conference on Computer and Communications Security, October 2003 .

[27] Donggang Liu, Peng Ning, and Wenliang Du. Group-based key pre-distribution in wireless sensor networks. Proceedings of ACM Workshop on Wireless Security, 2005.

[28] Konrad Lorincz, David J. Malan, Thaddeus R.F. Fulford-Jones, Alan Nawoj, Antony Clavel, Victor Shnayder, Geoffrey Mainland, Matt Welsh, and Steve Moulton. Sensor networks for emergency response: Challenges and opportunities. IEEE Pervasive Computing, pages 16-23, 2004.

[29] Alan Mainwaring, Joseph Polastre, Robert Szewczyk, David Culler, and John Anderson. Wireless sensor networks for habitat monitoring. ACM International Workshop on Wireless Sensor Networks and Applications, September 2002.

[30] DJ Malan, M Welsh, and MD Smith. A public-key infrastructure for key distribution in tinyos based on elliptic curve cryptography. Sensor and Ad Hoc Communications and Networks, 2004. IEEE SECON 2004. 2004 First Annual IEEE Communications Society Conference on, pages 71-80, 2004.

[31] J Newsome, E Shi, D Song, and A Perrig. The Sybil attack in sensor networks: analysis \& defenses. Proceedings of the third international symposium on Information processing in sensor networks, pages 259-268, 2004. 
[32] Taejoon Park and Kang G. Shin. Soft tamper-proofing via a program integrity verification in wireless sensor networks. IEEE Transactions on Mobile Computing, 4(3), 2005.

[33] A Perrig, J Stankovic, and D Wagner. Security in wireless sensor networks. Communications of the ACM, 47(6):53-57, 2004.

[34] Mahalingam Ramkumar. Safe renewal of a random key pre-distribution scheme for trusted devices. Proceedings of the IEEE Workshop on Information Assurance and Security, pages 142-149, 2005.

[35] Elaine Shi and Adrian Perrig. Designing secure sensor networks. IEEE Wireless Communications, December 2004.

[36] TinyOS. http://www.tinyos.net/.

[37] Patrick Traynor, Heesook Choi, Guohong Cao, Seneun Zhu, and Tom La Porta. Establishing pair-wise keys in heterogeneous sensor networks. 2004.

[38] AS Wander, N Gura, H Eberle, V Gupta, and SC Shantz. Energy analysis of public-key cryptography for wireless sensor networks. Third IEEE International Conference on Pervasive Computing and Communications (PERCOMÍ05), 2005.

[39] R Watro, D Kong, S Cuti, C Gardiner, C Lynn, and P Kruus. Tinypk: securing sensor networks with public key technology. Proceedings of the 2nd ACM workshop on Security of ad hoc and sensor networks, pages 59-64, 2004.

[40] R. Wei and J. Wu. Product construction of key distribution schemes for sensor networks. May 2004.

[41] Anthony D. Wood and John A. Stankovic. Denial of service in sensor networks. IEEE Computer, pages 54-62, 2002. 
[42] Qi Xue and Aura Ganz. Runtime security composition for sensor networks (securesense). IEEE Vehicular Technology Conference, 2003.

[43] Chee yee Chong and Srikanta P. Kumar. Sensor networks: Evolution, opportunities and challenges. Proceedings of the IEEE, 91(8), August 2003. 


\section{APPENDIX I \\ TinyOS System}

\subsection{TinyOS Installation}

The following procedure is the method used to install TinyOS.

1. Download and install the stable version of TinyOS from http://www.xbow.com/Support/Support_pdf_files/tinyos-1.1.0-1is.zip This will include all the necessary components for the Cygwin, TinyOS packages, TinyViz, and the special Crossbow libraries.

2. To launch the system, run the Cygwin shortcut on the desktop.

3. Navigate to the tinyos system bash $\$$ cd /opt/tinyos-1.x

4. You are now ready to use the TinyOS system.

\subsection{TinyViz}

TinyViz is installed with TinyOS. This is the simulator for running motes. In order to run a simulation, one must follow this procedure:

1. Build the application for the computer bash $\$$ make pc 
2. Enable the appropriate debug environment variables. For example:

bash $\$$ export DBG $=$ usr,usr2,usr3

3. Run main.exe and save the output to a trace file

$\begin{array}{ll}\text { bash } \$ \text { /opt/tinyos-1.x/tools/java/net/tinyos/sim/tinyviz } & \text {-run } \\ . / \text { build/pc/main.exe } 15 & \end{array}$

\subsection{Power Profiling}

1. Build the application

bash $\$$ make pc

2. Enable the power debug environment variable

bash $\$$ export $\mathrm{DBG}=$ power

3. Run main.exe and save the output to a trace file

$\operatorname{bash} \$ . /$ build $/$ pc/main.exe $-\mathrm{t}=60-\mathrm{p} 15$ i tree.trace

4. Run a post process script to compute the power consumption for each mote

$\operatorname{bash} \$ \quad /$ opt/tinyos-1.x/tools/scripts/PowerTOSSIM/postprocess.py $\quad-\mathrm{sb}=0$
-em /opt/tinyos-1.x/tools/scripts/PowerTOSSIM/mica2EnergyModel.txt
tree.trace

5. Single node example results which can be directly compared to other nodes to determine relative energy consumption. 
Mote 0, cpu total: 2224.706574

Mote 0, radio total: 3617.270259

Mote 0, adc total: 0.000000

Mote 0, leds total: 2892.881511

Mote 0, sensor total: 0.000000

Mote 0, eeprom total: 0.000000

Mote 0, cpucycle total: 0.000000

Mote 0, Total energy: 8734.858344 


\section{APPENDIX II}

\section{Testing}

\subsection{Key Generation Testing}

Results from 180 seconds of simulation with 2 mote sensors. This output shows the debug messages containing the packet data, the timer fired messages pushing data into the network, and the process of setting up the keys.

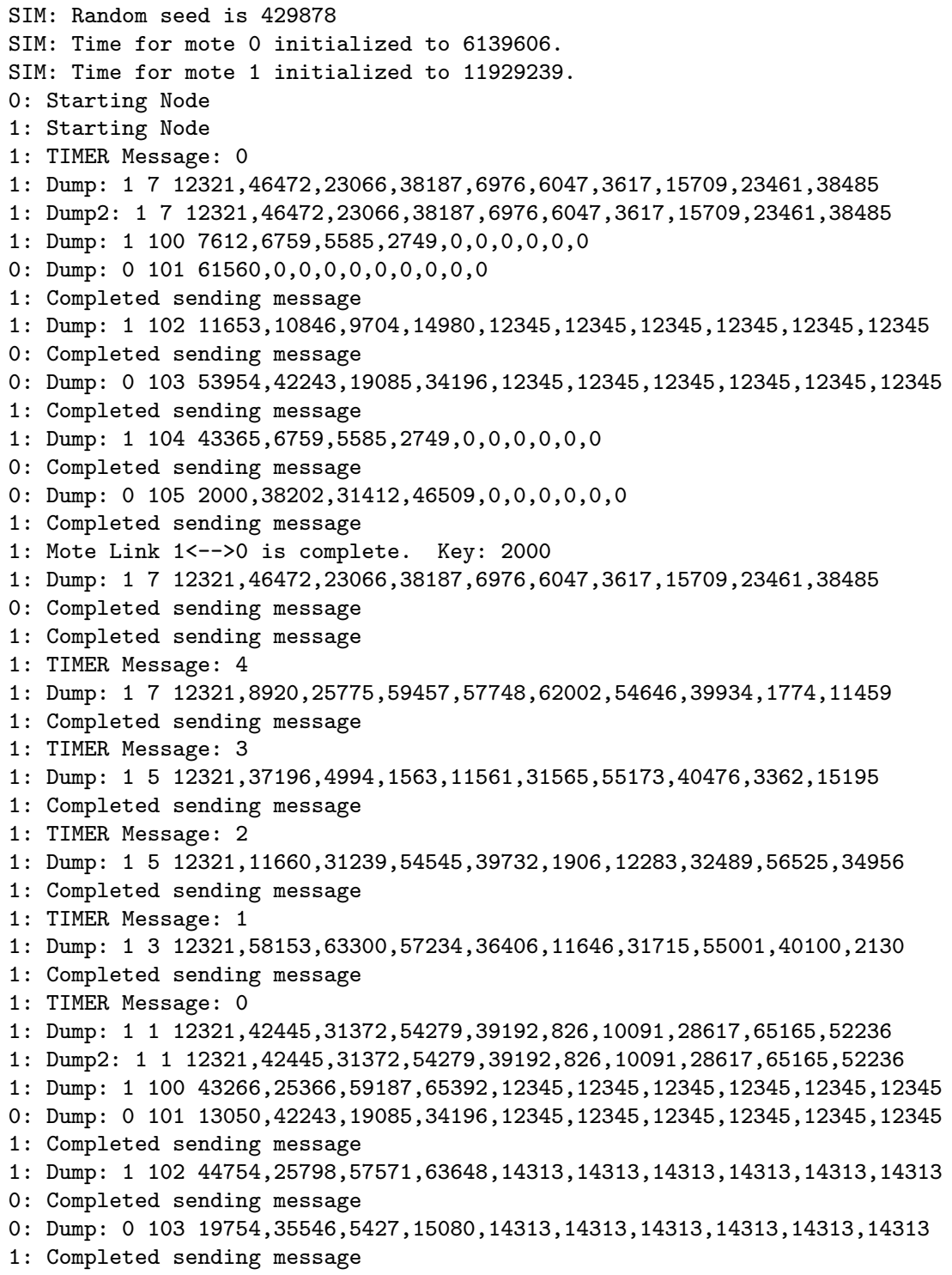


1: Dump: $110453584,25366,59187,65392,12345,12345,12345,12345,12345,12345$

0: Completed sending message

0: Dump: $010555858,36106,4835,15672,12345,12345,12345,12345,12345,12345$

1: Completed sending message

1: Mote Link $1<-->0$ is complete. Key: 55858

1: Dump: 11 12321,42445,31372,54279,39192,826,10091, 28617,65165,52236

0 : Completed sending message

1: Completed sending message

1: TIMER Message: 4

1: Dump: 10 12321,36810,11910,31763,55609,33636, 14290, 20155,48233, 18884

1: Completed sending message

1: TIMER Message: 3

1: Dump: $1012321,35183,9160,26255,60417,59668,58162,63350,57342,36590$

1: Completed sending message

1: TIMER Message: 2

1: Dump: 13 12321,53273,37156,4946,1979,11881,32205,55941,33820,14626

1: Completed sending message

1: TIMER Message: 1

1: Dump: 11 12321, 15940,23959, 39473,1396, 11255, 30449, 52477, 43244, 24770

1: Completed sending message

1: TIMER Message: 0

1: Dump: $1012321,53610,37830,5790,3107,14681,21421,34373,15772,23079$

1: Dump2: 10 12321,53610,37830,5790,3107, 14681, 21421, 34373, 15772, 23079

1: Dump: $110038225,7092,5751,3569,14313,14313,14313,14313,14313,14313$

0: Dump: 0101 20288,35546,5427,15080,14313,14313,14313,14313,14313,14313

1: Completed sending message

1: Dump: 1102 20323,49542,52293,55235,60891,60891,60891,60891,60891,60891

0 : Completed sending message

0: Dump: 0103 49684,33967,2533,836,60891,60891,60891,60891,60891,60891

1: Completed sending message

1: Dump: 1104 43004,7092,5751,3569,14313,14313,14313,14313,14313,14313

0 : Completed sending message

0: Dump: $010515490,24221,54231,55670,14313,14313,14313,14313,14313,14313$

1: Completed sending message

1: Mote Link $1<-->0$ is complete. Key: 15490

1: Dump: 10 12321,53610,37830,5790,3107,14681,21421,34373,15772, 23079

0 : Completed sending message

1: Completed sending message

1: TIMER Message: 4

1: Dump: $1712321,57809,62132,54386,39414,766,9443,26841,61613,53324$

1: Completed sending message

1: TIMER Message: 3

1: Dump: 16 12321,1331,11129,30701,52933,44188, 26658,61787, 54176, 38506

1: Completed sending message

1: TIMER Message: 2

1: Dump: $1312321,5177,2413,13253,18069,44085,27004,62439,55000,40122$

1: Completed sending message

1: TIMER Message: 1

1: Dump: 13 12321,17081,42093,31180,53895,37912,6458,4971,1993,11917

1: Completed sending message

1: TIMER Message: 0

1: Dump: $1512321,33596,14178,20443,48809,19524,47511,16952,42351,31688$

1: Dump2: $1512321,33596,14178,20443,48809,19524,47511,16952,42351,31688$

1: Dump: $110054927,39944,2330,13099,60891,60891,60891,60891,60891,60891$

0: Dump: $010155750,33967,2533,836,60891,60891,60891,60891,60891,60891$

1: Completed sending message

1: Dump: 1102 59917,41098,13720,4009,53593,53593,53593,53593,53593,53593

0 : Completed sending message

0: Dump: 0103 34739,4077,3924,3622,53593,53593,53593,53593,53593,53593

1: Completed sending message

1: Dump: 1104 3762,39944,2330,13099,60891,60891,60891,60891,60891,60891

0 : Completed sending message

0: Dump: 0105 2314,13167,13270,12964,60891,60891,60891,60891,60891,60891

1: Completed sending message

1: Mote Link $1<->0$ is complete. Key: 2314

1: Dump: 15 12321,33596,14178,20443,48809,19524,47511, 16952, 42351, 31688

0 : Completed sending message

1: Completed sending message 
1: TIMER Message: 4

1: Dump: 10 12321,42810,32614,57299,36528,11402,30731,53513,37636,5906

1: Completed sending message

1: TIMER Message: 3

1: Dump: 11 12321, 24525,40581,3100,14631, 21329, 34749, 15980, 24007, 39569

1: Completed sending message

Simulation of 2 motes completed.

\subsection{Network Communication Testing}

Results from 30 seconds of simulation with 5 mote sensors.

SIM: Random seed is 163419

4: Sent Message: (Node,value) 3,2

3: Received Message: (Node,value) 4,2

3: Sent Message: (Node, value) 2,2

1: Sent Message: (Node,value) 0,7

4: Sent Message: (Node,value) 3,6

3: Received Message: (Node,value) 4,6

3: Sent Message: (Node, value) 2,6

2: Received Message: (Node,value) 3,6

2: Sent Message: (Node, value) 1,6

1: Received Message: (Node,value) 2,6

1: Sent Message: (Node, value) 0,6

4: Sent Message: (Node, value) 3,4

3: Received Message: (Node,value) 4,4

3: Sent Message: (Node, value) 2,4

2: Received Message: (Node, value) 3,4

2: Sent Message: (Node, value) 1,4

1: Received Message: (Node, value) 2,4

1: Sent Message: (Node, value) 0,4

0: Received Message: (Node, value) 1,4

4: Sent Message: (Node, value) 3,4

3: Received Message: (Node,value) 4,4

3: Sent Message: (Node, value) 2,4

2: Received Message: (Node,value) 3,4

2: Sent Message: (Node, value) 1,4

1: Received Message: (Node,value) 2,4

1: Sent Message: (Node, value) 0,4

0: Received Message: (Node,value) 1,4

4: Sent Message: (Node, value) 3,1

3: Received Message: (Node,value) 4,1

3: Sent Message: (Node, value) 2,1

2: Received Message: (Node,value) 3,1

2: Sent Message: (Node, value) 1,1

1: Received Message: (Node,value) 2,1

1: Sent Message: (Node, value) 0,1

0: Received Message: (Node,value) 1,1

4: Sent Message: (Node, value) 3,0

3: Received Message: (Node,value) 4,0

3: Sent Message: (Node, value) 2,0

2: Received Message: (Node,value) 3,0

2: Sent Message: (Node, value) 1,0

1: Received Message: (Node,value) 2,0

1: Sent Message: (Node, value) 0,0

0: Received Message: (Node,value) 1,0

4: Sent Message: (Node, value) 3,7

3: Received Message: (Node, value) 4,7

3: Sent Message: (Node, value) 2,7

2: Received Message: (Node,value) 3,7

2: Sent Message: (Node, value) 1,7

1: Received Message: (Node,value) 2,7

1: Sent Message: (Node, value) 0,7

0 : Received Message: (Node, value) 1,7 
4: Sent Message: (Node, value) 3,2

3: Received Message: (Node, value) 4,2

3: Sent Message: (Node, value) 2,2

2: Received Message: (Node, value) 3,2

2: Sent Message: (Node, value) 1,2

1: Received Message: (Node,value) 2,2

1: Sent Message: (Node, value) 0,2

0: Received Message: (Node,value) 1,2

4: Sent Message: (Node,value) 3,1

3: Received Message: (Node,value) 4,1

3: Sent Message: (Node, value) 2,1

2: Received Message: (Node,value) 3,1

2: Sent Message: (Node, value) 1,1

1: Received Message: (Node,value) 2,1

1: Sent Message: (Node, value) 0,1

0 : Received Message: (Node,value) 1,1

Simulation of 5 motes completed.

\subsection{Sample Tree Application Test}

Results from 30 seconds of simulation with 15 mote sensors.

SIM: Random seed is 505942

SIM: Time for mote 0 initialized to 38308441.

SIM: Time for mote 1 initialized to 11063463.

SIM: Time for mote 2 initialized to 37427157.

SIM: Time for mote 3 initialized to 6269218.

SIM: Time for mote 4 initialized to 7424788 .

SIM: Time for mote 5 initialized to 21263943.

SIM: Time for mote 6 initialized to 30998662 .

SIM: Time for mote 7 initialized to 26766772 .

SIM: Time for mote 8 initialized to 37219119.

SIM: Time for mote 9 initialized to 793060 .

SIM: Time for mote 10 initialized to 9022949.

SIM: Time for mote 11 initialized to 366235.

SIM: Time for mote 12 initialized to 13551507.

SIM: Time for mote 13 initialized to 35265355.

SIM: Time for mote 14 initialized to 28527150.

11: Starting Node

11: Starting Timer

9: Starting Node

9: Starting Timer

3: Starting Node

3: Starting Timer

4: Starting Node

4: Starting Timer

10: Starting Node

10: Starting Timer

1: Starting Node

1: Starting Timer

12: Starting Node

12: Starting Timer

5: Starting Node

5: Starting Timer

7: Starting Node

7: Starting Timer

14: Starting Node

14: Starting Timer

6: Starting Node

6: Starting Timer

13: Starting Node

13: Starting Timer

8: Starting Node

8: Starting Timer 


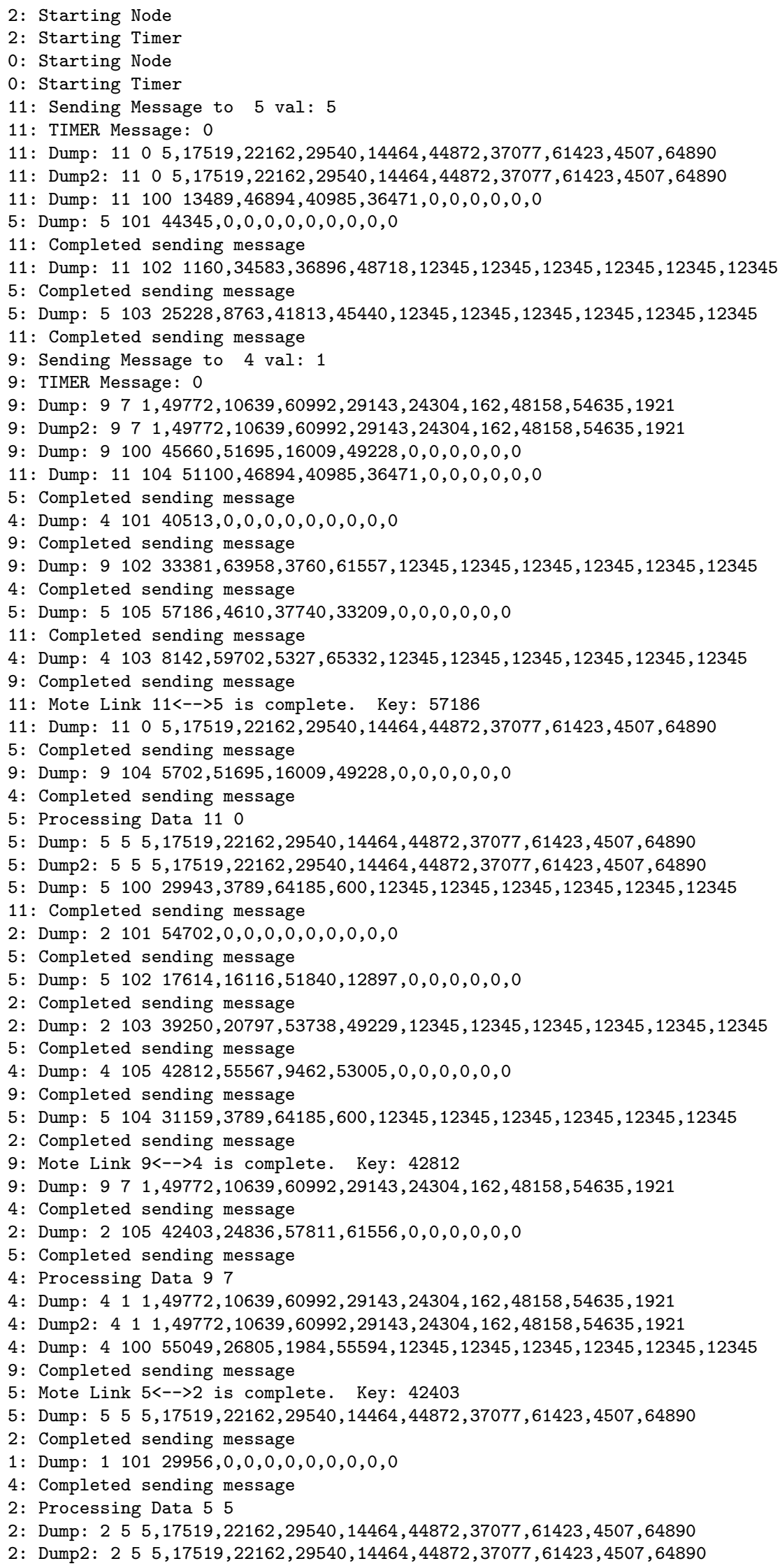


2: Dump: $21008742,14300,7208,19392,12345,12345,12345,12345,12345,12345$

5: Completed sending message

4: Dump: $410259184,22668,14329,59667,0,0,0,0,0,0$

1: Completed sending message

0: Dump: $010122049,0,0,0,0,0,0,0,0,0$

2: Completed sending message

1: Dump: $110346472,23066,38187,6976,12345,12345,12345,12345,12345,12345$

4: Completed sending message

2: Dump: $21024639,2021,11281,31737,0,0,0,0,0,0$

0 : Completed sending message

4: Dump: $410420097,26805,1984,55594,12345,12345,12345,12345,12345,12345$

1: Completed sending message

o: Dump: $010353954,42243,19085,34196,12345,12345,12345,12345,12345,12345$

2: Completed sending message

2: Dump: 2104 43365,14300,7208,19392,12345,12345,12345,12345,12345,12345

0 : Completed sending message

1: Dump: $11053617,27171,42258,11129,0,0,0,0,0,0$

4: Completed sending message

4: Mote Link $4<-->1$ is complete. Key: 3617

4: Dump: 41 1,49772,10639,60992,29143,24304,162,48158,54635, 1921

1: Completed sending message

1: Processing Data 41

1: Dump: 11 1,49772,10639,60992, 29143, 24304,162,48158,54635, 1921

1: Dump2: 11 1,49772,10639,60992,29143, 24304,162,48158,54635, 1921

1: Dump: 1100 23461,38485,7612,6759,12345,12345,12345,12345,12345,12345

4: Completed sending message

0: Dump: $010142028,38202,31412,46509,0,0,0,0,0,0$

1: Completed sending message

0: Dump: $01057982,42243,19085,34196,12345,12345,12345,12345,12345,12345$

2: Completed sending message

1: Mote Link $1<-->0$ is complete. Key: 7982

1: Dump: 11 1,49772,10639,60992, 29143, 24304,162, 48158, 54635, 1921

0: Completed sending message

0 : Processing Data 11

1: Completed sending message

3: Sending Message to 1 val: 0

3: TIMER Message: 0

3: Dump: 30 0,46109,14862,13857,11903,7875, 32699, 48459, 10402, 4985

3: Dump2: 3 o 0,46109,14862,13857,11903,7875,32699,48459,10402, 4985

3: Dump: $310025807,35747,17778,51417,0,0,0,0,0,0$

1: Dump: $110147081,42604,11653,10846,0,0,0,0,0,0$

3: Completed sending message

3: Dump: 3102 21750,48026,30027,63712,12345,12345,12345, 12345, 12345, 12345

1: Completed sending message

1: Dump: 1103 18901,45749,21628,35303,12345, 12345, 12345, 12345, 12345, 12345

3: Completed sending message

3: Dump: $310449588,35747,17778,51417,0,0,0,0,0,0$

1: Completed sending message

1: Dump: 1105 25775,33420,25669,47582,0,0,0,0,0,0

3: Completed sending message

3: Mote Link $3<-->1$ is complete. Key: 25775

3: Dump: 30 0,46109,14862,13857, 11903,7875, 32699, 48459, 10402, 4985

1: Completed sending message

1: Processing Data 30

1: Dump: 11 0,46109,14862,13857,11903,7875,32699,48459,10402, 4985

3: Completed sending message

0 : Processing Data 11

1: Completed sending message

10: Sending Message to $4 \mathrm{val}: 1$

10: TIMER Message: 0

10: Dump: $1001,39295,52518,26004,9465,42543,45958,39124,52848,25400$

10: Dump2: $1001,39295,52518,26004,9465,42543,45958,39124,52848,25400$

10: Dump: $1010010657,48287,34534,61972,0,0,0,0,0,0$

4: Dump: $41018299,22668,14329,59667,0,0,0,0,0,0$

10: Completed sending message

10: Dump: $101026552,36006,46815,49709,12345,12345,12345,12345,12345,12345$

4: Completed sending message

4: Dump: 4103 63072,10851,33388,49787,12345,12345,12345,12345,12345, 12345 
10: Completed sending message

10: Dump: $101046738,48287,34534,61972,0,0,0,0,0,0$

4: Completed sending message

4: Dump: 4105 21048,6746,45653,62018,0,0,0,0,0,0

10: Completed sending message

10: Mote Link $10<-->4$ is complete. Key: 21048

10: Dump: $1001,39295,52518,26004,9465,42543,45958,39124,52848,25400$

4: Completed sending message

4: Processing Data 100

4: Dump: 411 1,39295,52518, 26004, 9465, 42543, 45958, 39124, 52848, 25400

10: Completed sending message

1: Processing Data 41

1: Dump: 11 1,39295, 52518, 26004, 9465, 42543, 45958, 39124, 52848, 25400

4: Completed sending message

0: Processing Data 11

1: Completed sending message

12: Sending Message to 5 val: 1

12: TIMER Message: 0

12: Dump: $1231,53536,63490,43590,3790,22487,58857,37264,31074,47247$

12: Dump2: $1231,53536,63490,43590,3790,22487,58857,37264,31074,47247$

12: Dump: $1210011100,7411,29617,44333,0,0,0,0,0,0$

5: Dump: $510122776,16116,51840,12897,0,0,0,0,0,0$

12: Completed sending message

12: Dump: 12102 7013,11466,17288,40212,12345,12345,12345,12345,12345, 12345

5: Completed sending message

5: Dump: 5103 60479,12116,47499,33852,12345,12345,12345,12345,12345, 12345

12: Completed sending message

12: Dump: $1210419124,7411,29617,44333,0,0,0,0,0,0$

5: Completed sending message

5: Dump: $51052482,8045,35250,46085,0,0,0,0,0,0$

12: Completed sending message

12: Mote Link $12<-->5$ is complete. Key: 2482

12: Dump: 123 1,53536,63490,43590,3790, 22487, 58857,37264, 31074, 47247

5: Completed sending message

5: Processing Data 123

5: Dump: $551,53536,63490,43590,3790,22487,58857,37264,31074,47247$

12: Completed sending message

2: Processing Data 55

2: Dump: 21 1,53536,63490,43590,3790, 22487, 58857, $37264,31074,47247$

2: Dump2: $211,53536,63490,43590,3790,22487,58857,37264,31074,47247$

2: Dump: $210064968,38921,21383,54430,0,0,0,0,0,0$

5: Completed sending message

0: Dump: $010115557,38202,31412,46509,0,0,0,0,0,0$

2: Completed sending message

0: Completed sending message

7: Sending Message to 3 val: 1

7: TIMER Message: 0

7: Dump: 74 1,63570,25896,20009,6187,46127,64558,27680, 23609,15371

7: Dump2: 74 1,63570,25896,20009,6187,46127,64558, 27680, 23609,15371

7: Dump: $710064623,27822,23845,15923,0,0,0,0,0,0$

3: Dump: $310154402,48026,30027,63712,12345,12345,12345,12345,12345,12345$

7: Completed sending message

7: Dump: 7102 52310,23703,27932,3594,12345,12345,12345,12345,12345,12345

3: Completed sending message

3: Dump: $310327997,39047,25402,33865,0,0,0,0,0,0$

7: Completed sending message

7: Dump: 7104 49604,27822,23845,15923,0,0,0,0,0,0

3: Completed sending message

3: Dump: 3105 63345,43198,21251,46192,12345,12345,12345,12345,12345, 12345

7: Completed sending message

7: Mote Link $7<-->3$ is complete. Key: 63345

7: Dump: 74 1,63570,25896,20009,6187,46127,64558,27680, 23609, 15371

3: Completed sending message

3: Processing Data 74

3: Dump: 31 1,63570, 25896, 20009,6187,46127,64558, 27680, 23609, 15371

7: Completed sending message

1: Processing Data 31

1: Dump: 11 1,63570,25896,20009,6187,46127,64558,27680,23609,15371 


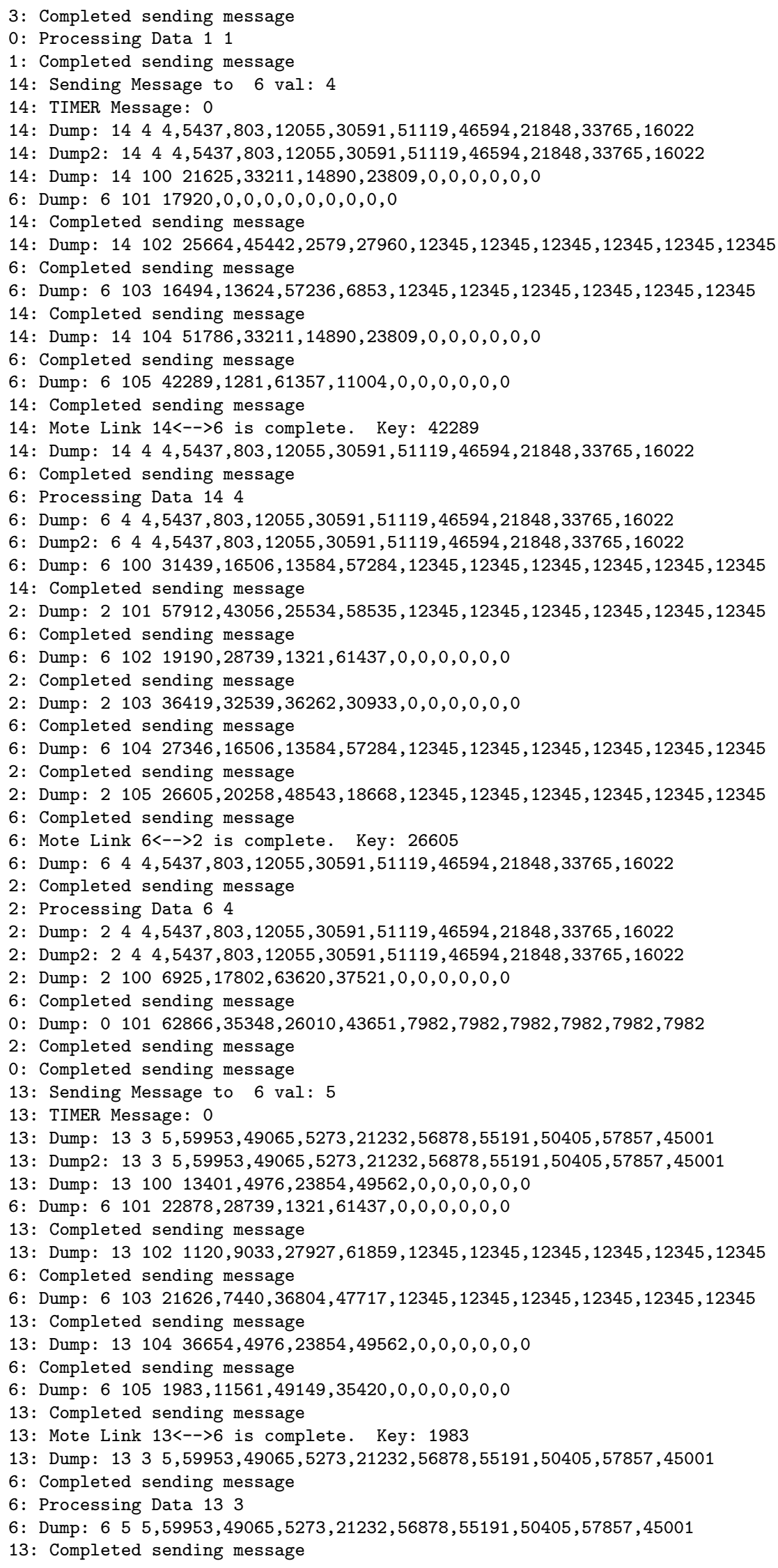


2: Processing Data 65

2: Dump: 25 5,59953,49065,5273,21232,56878,55191,50405,57857,45001

2: Dump2: 25 5,59953,49065,5273,21232,56878,55191,50405,57857,45001

2: Dump: 2100 65278,40549,24431,52558,12345,12345, 12345,12345, 12345, 12345

6: Completed sending message

0: Dump: $010135801,38202,31412,46509,0,0,0,0,0,0$

2: Completed sending message

0 : Completed sending message

8: Sending Message to 3 val: 5

8: TIMER Message: 0

8: Dump: $875,29857,26041,18313,1001,35625,35492,35262,36746,33762$

8: Dump2: $875,29857,26041,18313,1001,35625,35492,35262,36746,33762$

8: Dump: $810039730,43666,51666,3922,0,0,0,0,0,0$

3: Dump: $310156874,39047,25402,33865,0,0,0,0,0,0$

8: Completed sending message

8: Dump: 8102 43787,39595,63979,16235,12345,12345,12345,12345,12345,12345

3: Completed sending message

3: Dump: 3103 26711,37523,30482,44057,12345,12345,12345,12345,12345, 12345

8: Completed sending message

8: Dump: $810458230,43666,51666,3922,0,0,0,0,0,0$

3: Completed sending message

3: Dump: $310522065,41642,18219,39968,0,0,0,0,0,0$

8: Completed sending message

8: Mote Link $8<-->3$ is complete. Key: 22065

8: Dump: 87 5,29857, 26041, 18313, 1001, 35625, 35492, 35262, 36746, 33762

3: Completed sending message

3: Processing Data 87

3: Dump: 35 5,29857, 26041, 18313, 1001, 35625, 35492, 35262, 36746, 33762

8: Completed sending message

1: Processing Data 35

1: Dump: 15 5,29857,26041,18313,1001,35625,35492,35262, 36746, 33762

3: Completed sending message

0 : Processing Data 15

1: Completed sending message

11: Sending Message to 5 val: 4

11: TIMER Message: 4

11: Dump: 116 4,33008,53157,20751,31826,9956,37760,59717,7375,59346

5: Processing Data 116

5: Dump: 54 4,33008,53157,20751,31826,9956,37760,59717, 7375, 59346

11: Completed sending message

2: Processing Data 54

2: Dump: $254,33008,53157,20751,31826,9956,37760,59717,7375,59346$

2: Dump2: 25 4,33008,53157,20751,31826,9956,37760,59717,7375,59346

2: Dump: $210037295,16579,61974,34741,0,0,0,0,0,0$

5: Completed sending message

0: Dump: $010127615,35348,26010,43651,7982,7982,7982,7982,7982,7982$

2: Completed sending message

9: Sending Message to 4 val: 3

9: TIMER Message: 4

9: Dump: 96 3,45672,51591,15961,49644,11919,57408, 28119, 26352, 28834

0 : Completed sending message

4: Processing Data 96

4: Dump: 43 3,45672,51591,15961,49644,11919,57408, 28119, 26352, 28834

9: Completed sending message

1: Processing Data 43

1: Dump: 15 3,45672,51591,15961,49644,11919,57408, 28119, 26352, 28834

1: Dump2: 15 3,45672,51591,15961,49644,11919,57408,28119,26352,28834

1: Dump: $11001774,11459,30873,53293,12345,12345,12345,12345,12345,12345$

4: Completed sending message

0: Dump: $010131456,38202,31412,46509,0,0,0,0,0,0$

1: Completed sending message

0 : Completed sending message

10: Sending Message to 4 val: 3

10: TIMER Message: 4

10: Dump: 107 3,6766,56077, 18882,31829,5991,49435, 32238, 5133,50999

4: Processing Data 107

4: Dump: 43 3,6766,56077, 18882,31829,5991, 49435, 32238, 5133, 50999

10: Completed sending message 
1: Processing Data 43

1: Dump: 15 3,6766,56077,18882,31829,5991,49435,32238,5133,50999

1: Dump2: 15 3,6766,56077,18882,31829,5991,49435, 32238, 5133,50999

1: Dump: 1100 4994,1563,11561,31565,12055,12055,12055,12055,12055, 12055

4: Completed sending message

0: Dump: $010153918,42243,19085,34196,12345,12345,12345,12345,12345,12345$

1: Completed sending message

0 : Completed sending message

12: Sending Message to 5 val: 6

12: TIMER Message: 4

12: Dump: 122 6,41415,6604,31187,47601,10656,6411,30817, 47757,12120

5: Processing Data 122

5: Dump: $566,41415,6604,31187,47601,10656,6411,30817,47757,12120$

12: Completed sending message

2: Processing Data 56

2: Dump: $266,41415,6604,31187,47601,10656,6411,30817,47757,12120$

2: Dump2: 26 6,41415,6604,31187,47601,10656,6411,30817,47757,12120

2: Dump: 2100 43918,13445,6810,18084,12345,12345, 12345, 12345, 12345, 12345

5: Completed sending message

0: Dump: $010155757,38202,31412,46509,0,0,0,0,0,0$

2: Completed sending message

0 : Completed sending message

7: Sending Message to 3 val: 6

7: TIMER Message: 4

7: Dump: 77 6,16608,1465, 36619, 35430, 32944, 38164, 48732, 59596, 17900

3: Processing Data 77

3: Dump: 36 6,16608,1465, 36619, 35430, 32944, 38164, 48732, 59596, 17900

7: Completed sending message

1: Processing Data 36

1: Dump: $166,16608,1465,36619,35430,32944,38164,48732,59596,17900$

1: Dump2: 16 6,16608,1465,36619,35430,32944,38164,48732,59596, 17900

1: Dump: $110040476,3362,15195,22441,12345,12345,12345,12345,12345,12345$

3: Completed sending message

0: Dump: $010116126,35348,26010,43651,7982,7982,7982,7982,7982,7982$

1: Completed sending message

0 : Completed sending message

14: Sending Message to $6 \mathrm{val}: 7$

14: TIMER Message: 4

14: Dump: $1477,61935,55938,35928,8684,27277,64579,49626,47848,19596$

6: Processing Data 147

6: Dump: $677,61935,55938,35928,8684,27277,64579,49626,47848,19596$

14: Completed sending message

2: Processing Data 67

2: Dump: $277,61935,55938,35928,8684,27277,64579,49626,47848,19596$

2: Dump2: $277,61935,55938,35928,8684,27277,64579,49626,47848,19596$

2: Dump: $210040489,24519,52254,64421,0,0,0,0,0,0$

6: Completed sending message

0: Dump: $010154822,47661,21923,39610,12055,12055,12055,12055,12055,12055$

2: Completed sending message

0 : Completed sending message

13: Sending Message to $6 \mathrm{val}: 7$

13: TIMER Message: 4

13: Dump: $1367,30565,38152,16859,63604,39715,23949,49368,60027,48957$

6: Processing Data 136

6: Dump: $677,30565,38152,16859,63604,39715,23949,49368,60027,48957$

13: Completed sending message

2: Processing Data 67

2: Dump: $277,30565,38152,16859,63604,39715,23949,49368,60027,48957$

2: Dump2: $277,30565,38152,16859,63604,39715,23949,49368,60027,48957$

2: Dump: 2100 19011,59158,44469,14351,12345,12345,12345, 12345, 12345, 12345

6: Completed sending message

0: Dump: 0101 24799,42243,19085,34196,12345,12345,12345, 12345, 12345, 12345

2: Completed sending message

0 : Completed sending message

8: Sending Message to 3 val: 5

8: TIMER Message: 4

8: Dump: 83 5,38460,45198,65002, 26402,17083, 2437, 40945, 41748,56030

3: Processing Data 83 
3: Dump: 36 5,38460,45198,65002, 26402,17083, 2437, 40945, 41748,56030

8: Completed sending message

1: Processing Data 36

1: Dump: 15 5,38460,45198,65002, 26402,17083, 2437, 40945, 41748, 56030

1: Dump2: 15 5,38460,45198,65002,26402,17083, 2437,40945, 41748,56030

1: Dump: 1100 11660,31239,54545,39732,12055,12055,12055,12055,12055, 12055

3: Completed sending message

0: Dump: $01019540,47661,21923,39610,12055,12055,12055,12055,12055,12055$

1: Completed sending message

0 : Completed sending message

11: Sending Message to 5 val: 1

11: TIMER Message: 3

11: Dump: $1161,13566,47020,41245,35967,54971,25395,6186,60948,4717$

5: Processing Data 116

5: Dump: $561,13566,47020,41245,35967,54971,25395,6186,60948,4717$

11: Completed sending message

2: Processing Data 56

2: Dump: $271,13566,47020,41245,35967,54971,25395,6186,60948,4717$

2: Dump2: $271,13566,47020,41245,35967,54971,25395,6186,60948,4717$

2: Dump: $210029836,39560,22153,56962,0,0,0,0,0,0$

5: Completed sending message

0: Dump: $010125712,35348,26010,43651,7982,7982,7982,7982,7982,7982$

2: Completed sending message

9: Sending Message to 4 val: 6

9: TIMER Message: 3

9: Dump: 96 6,49787,10657,60956,29039,24448,578,47582,57067,4225

0 : Completed sending message

4: Processing Data 96

4: Dump: 46 6,49787,10657,60956, 29039, 24448,578,47582,57067, 4225

9: Completed sending message

1: Processing Data 46

1: Dump: $166,49787,10657,60956,29039,24448,578,47582,57067,4225$

1: Dump2: 16 6,49787,10657,60956,29039,24448,578,47582,57067,4225

1: Dump: 1100 12283,32489,56525,34956,12345, 12345, 12345, 12345, 12345, 12345

4: Completed sending message

0: Dump: $010164437,38202,31412,46509,0,0,0,0,0,0$

1: Completed sending message

0 : Completed sending message

Simulation of 15 motes completed.

\subsection{Base Tree Power Profile}

Results from 180 seconds of simulation with 15 mote sensors

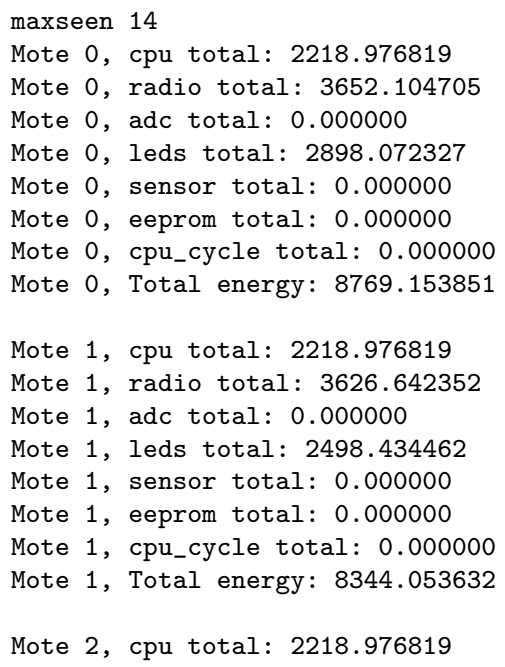

Mote 2, cpu total: 2218.976819 
Mote 2, radio total: 3609.511452

Mote 2, adc total: 0.000000

Mote 2, leds total: 2548.352690

Mote 2, sensor total: 0.000000

Mote 2, eeprom total: 0.000000

Mote 2, cpu_cycle total: 0.000000

Mote 2, Total energy: 8376.840962

Mote 3, cpu total: 2218.976819

Mote 3, radio total: 3629.881890

Mote 3, adc total: 0.000000

Mote 3, leds total: 1973.682531

Mote 3, sensor total: 0.000000

Mote 3, eeprom total: 0.000000

Mote 3, cpu_cycle total: 0.000000

Mote 3, Total energy: 7822.541240

Mote 4, cpu total: 2218.976819

Mote 4, radio total: 3715.867709

Mote 4, adc total: 0.000000

Mote 4, leds total: 2226.251559

Mote 4, sensor total: 0.000000

Mote 4, eeprom total: 0.000000

Mote 4, cpu_cycle total: 0.000000

Mote 4, Total energy: 8161.096087

Mote 5, cpu total: 2218.976819

Mote 5, radio total: 3634.872872

Mote 5, adc total: 0.000000

Mote 5, leds total: 2190.444029

Mote 5, sensor total: 0.000000

Mote 5, eeprom total: 0.000000

Mote 5, cpu_cycle total: 0.000000

Mote 5, Total energy: 8044.293721

Mote 6, cpu total: 2218.976819

Mote 6 , radio total: 3761.738920

Mote 6 , adc total: 0.000000

Mote 6, leds total: 2174.156711

Mote 6, sensor total: 0.000000

Mote 6, eeprom total: 0.000000

Mote 6, cpu_cycle total: 0.000000

Mote 6, Total energy: 8154.872450

Mote 7, cpu total: 2218.976819

Mote 7 , radio total: 3672.689758

Mote 7 , adc total: 0.000000

Mote 7, leds total: 1578.147064

Mote 7, sensor total: 0.000000

Mote 7, eeprom total: 0.000000

Mote 7, cpu_cycle total: 0.000000

Mote 7, Total energy: 7469.813641

Mote 8, cpu total: 2218.976819

Mote 8 , radio total: 3785.418183

Mote 8, adc total: 0.000000

Mote 8, leds total: 1532.638110

Mote 8, sensor total: 0.000000

Mote 8, eeprom total: 0.000000

Mote 8, cpu_cycle total: 0.000000

Mote 8, Total energy: 7537.033112

Mote 9, cpu total: 2202.844661

Mote 9, radio total: 3757.922072

Mote 9, adc total: 0.000000

Mote 9, leds total: 1618.119804

Mote 9, sensor total: 0.000000

Mote 9, eeprom total: 0.000000 
Mote 9, cpu_cycle total: 0.000000

Mote 9, Total energy: 7578.886537

Mote 10, cpu total: 2150.746170

Mote 10 , radio total: 3616.119656

Mote 10, adc total: 0.000000

Mote 10, leds total: 1774.790384

Mote 10, sensor total: 0.000000

Mote 10, eeprom total: 0.000000

Mote 10, cpu_cycle total: 0.000000

Mote 10, Total energy: 7541.656211

Mote 11, cpu total: 2150.746170

Mote 11 , radio total: 3594.948287

Mote 11, adc total: 0.000000

Mote 11, leds total: 1722.899798

Mote 11, sensor total: 0.000000

Mote 11, eeprom total: 0.000000

Mote 11, cpu_cycle total: 0.000000

Mote 11, Total energy: 7468.594255

Mote 12, cpu total: 2150.746170

Mote 12 , radio total: 3665.030092

Mote 12, adc total: 0.000000

Mote 12, leds total: 1940.712262

Mote 12, sensor total: 0.000000

Mote 12, eeprom total: 0.000000

Mote 12, cpu_cycle total: 0.000000

Mote 12, Total energy: 7756.488524

Mote 13, cpu total: 2150.746170

Mote 13, radio total: 3654.548687

Mote 13, adc total: 0.000000

Mote 13, leds total: 1605.464870

Mote 13, sensor total: 0.000000

Mote 13, eeprom total: 0.000000

Mote 13, cpu_cycle total: 0.000000

Mote 13, Total energy: 7410.759727

Mote 14, cpu total: 2150.746170

Mote 14, radio total: 3669.187537

Mote 14, adc total: 0.000000

Mote 14, leds total: 1648.371681

Mote 14, sensor total: 0.000000

Mote 14, eeprom total: 0.000000

Mote 14, cpu_cycle total: 0.000000

Mote 14, Total energy: 7468.305388

\subsection{New Tree Power Profile}

Results from 180 seconds of simulation with 15 mote sensors

maxseen 14

Mote 0, cpu total: 2203.959851

Mote 0, radio total: 3696.191106

Mote 0, adc total: 0.000000

Mote 0, leds total: 2222.547375

Mote 0, sensor total: 0.000000

Mote 0, eeprom total: 0.000000

Mote 0, cpu_cycle total: 0.000000

Mote 0, Total energy: 8122.698332

Mote 1, cpu total: 2203.959851

Mote 1 , radio total: 3732.661747 
Mote 1, adc total: 0.000000

Mote 1, leds total: 2052.241901

Mote 1, sensor total: 0.000000

Mote 1, eeprom total: 0.000000

Mote 1, cpu_cycle total: 0.000000

Mote 1, Total energy: 7988.863498

Mote 2, cpu total: 2203.959851

Mote 2, radio total: 3700.232011

Mote 2, adc total: 0.000000

Mote 2, leds total: 2269.787883

Mote 2, sensor total: 0.000000

Mote 2, eeprom total: 0.000000

Mote 2, cpu_cycle total: 0.000000

Mote 2, Total energy: 8173.979745

Mote 3, cpu total: 2203.959851

Mote 3, radio total: 3762.596357

Mote 3, adc total: 0.000000

Mote 3, leds total: 1986.239145

Mote 3, sensor total: 0.000000

Mote 3, eeprom total: 0.000000

Mote 3, cpu_cycle total: 0.000000

Mote 3, Total energy: 7952.795352

Mote 4, cpu total: 2203.943069

Mote 4, radio total: 3690.571027

Mote 4, adc total: 0.000000

Mote 4, leds total: 2202.636157

Mote 4, sensor total: 0.000000

Mote 4, eeprom total: 0.000000

Mote 4, cpu_cycle total: 0.000000

Mote 4, Total energy: 8097.150252

Mote 5, cpu total: 2203.943069

Mote 5, radio total: 3671.221505

Mote 5, adc total: 0.000000

Mote 5, leds total: 2089.842176

Mote 5, sensor total: 0.000000

Mote 5, eeprom total: 0.000000

Mote 5, cpu_cycle total: 0.000000

Mote 5, Total energy: 7965.006750

Mote 6, cpu total: 2203.943069

Mote 6, radio total: 3762.733678

Mote 6, adc total: 0.000000

Mote 6, leds total: 2468.257579

Mote 6, sensor total: 0.000000

Mote 6, eeprom total: 0.000000

Mote 6, cpu_cycle total: 0.000000

Mote 6, Total energy: 8434.934325

Mote 7, cpu total: 2197.774589

Mote 7 , radio total: 3668.898956

Mote 7 , adc total: 0.000000

Mote 7 , leds total: 1274.993334

Mote 7, sensor total: 0.000000

Mote 7 , eeprom total: 0.000000

Mote 7, cpu_cycle total: 0.000000

Mote 7 , Total energy: 7141.666880

Mote 8, cpu total: 2197.774589

Mote 8 , radio total: 3723.208888

Mote 8, adc total: 0.000000

Mote 8, leds total: 1749.073120

Mote 8, sensor total: 0.000000

Mote 8, eeprom total: 0.000000

Mote 8, cpu_cycle total: 0.000000 
Mote 8, Total energy: 7670.056597

Mote 9, cpu total: 2197.774589

Mote 9 , radio total: 3720.864808

Mote 9 , adc total: 0.000000

Mote 9, leds total: 1551.275613

Mote 9, sensor total: 0.000000

Mote 9, eeprom total: 0.000000

Mote 9, cpu_cycle total: 0.000000

Mote 9, Total energy: 7469.915010

Mote 10, cpu total: 2197.774589

Mote 10 , radio total: 3632.346702

Mote 10, adc total: 0.000000

Mote 10, leds total: 1714.697507

Mote 10, sensor total: 0.000000

Mote 10, eeprom total: 0.000000

Mote 10, cpu_cycle total: 0.000000

Mote 10, Total energy: 7544.818798

Mote 11, cpu total: 2197.774589

Mote 11 , radio total: 3631.745911

Mote 11 , adc total: 0.000000

Mote 11, leds total: 1663.282477

Mote 11, sensor total: 0.000000

Mote 11, eeprom total: 0.000000

Mote 11, cpu_cycle total: 0.000000

Mote 11, Total energy: 7492.802977

Mote 12, cpu total: 2197.774589

Mote 12 , radio total: 3575.857780

Mote 12, adc total: 0.000000

Mote 12, leds total: 1568.121608

Mote 12, sensor total: 0.000000

Mote 12, eeprom total: 0.000000

Mote 12, cpu_cycle total: 0.000000

Mote 12, Total energy: 7341.753977

Mote 13, cpu total: 2197.774589

Mote 13 , radio total: 3730.243473

Mote 13, adc total: 0.000000

Mote 13, leds total: 1943.839616

Mote 13, sensor total: 0.000000

Mote 13 , eeprom total: 0.000000

Mote 13, cpu_cycle total: 0.000000

Mote 13, Total energy: 7871.857679

Mote 14, cpu total: 2197.774589

Mote 14 , radio total: 3747.671901

Mote 14, adc total: 0.000000

Mote 14, leds total: 1954.747879

Mote 14, sensor total: 0.000000

Mote 14, eeprom total: 0.000000

Mote 14, cpu_cycle total: 0.000000

Mote 14, Total energy: 7900.194369 


\section{APPENDIX III \\ Source Code}

\subsection{Network Test Application}

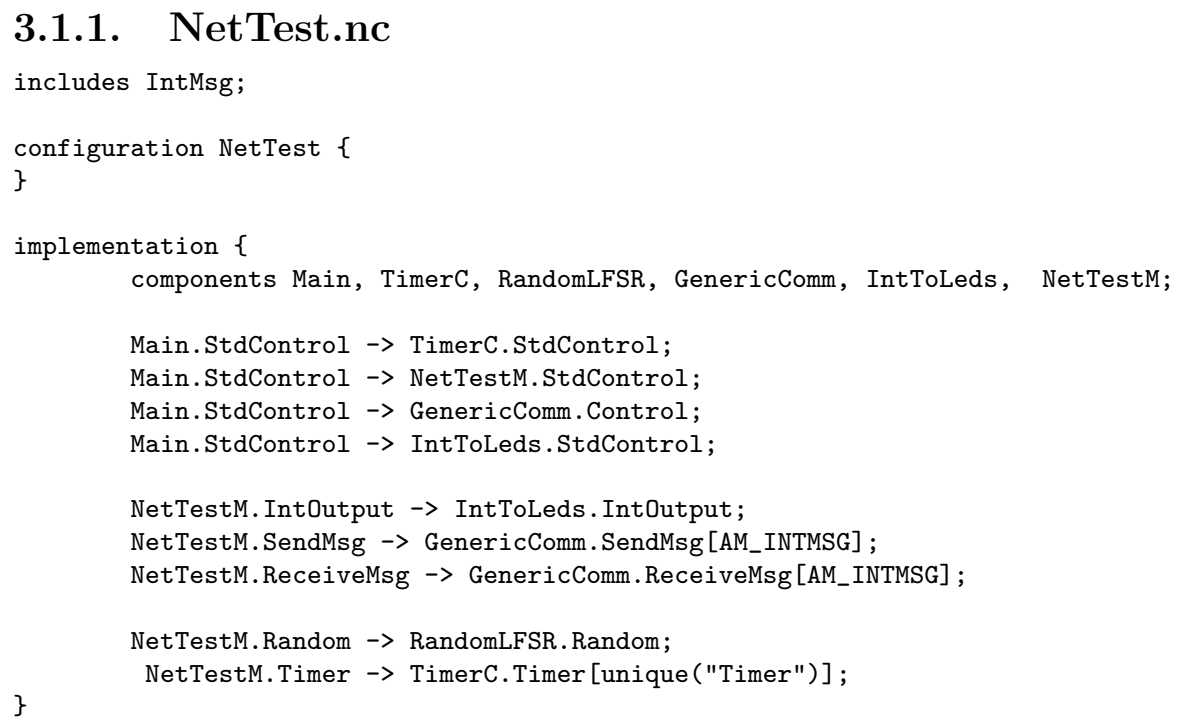

\subsubsection{NetTestM.nc}

includes IntMsg;

module NetTestM \{

provides \{

interface StdControl;

\}

uses \{

interface SendMsg;

interface ReceiveMsg;

interface IntOutput;

interface Random;

\}

interface Timer;

\}

implementation \{

struct TOS_Msg data;

uint16_t receiveFlag;

uint16_t leftNode;

uint16_t rightNode;

//// Interface $/ / / / /$

$/ / / / / / / / / / / / / / / / / / /$ 


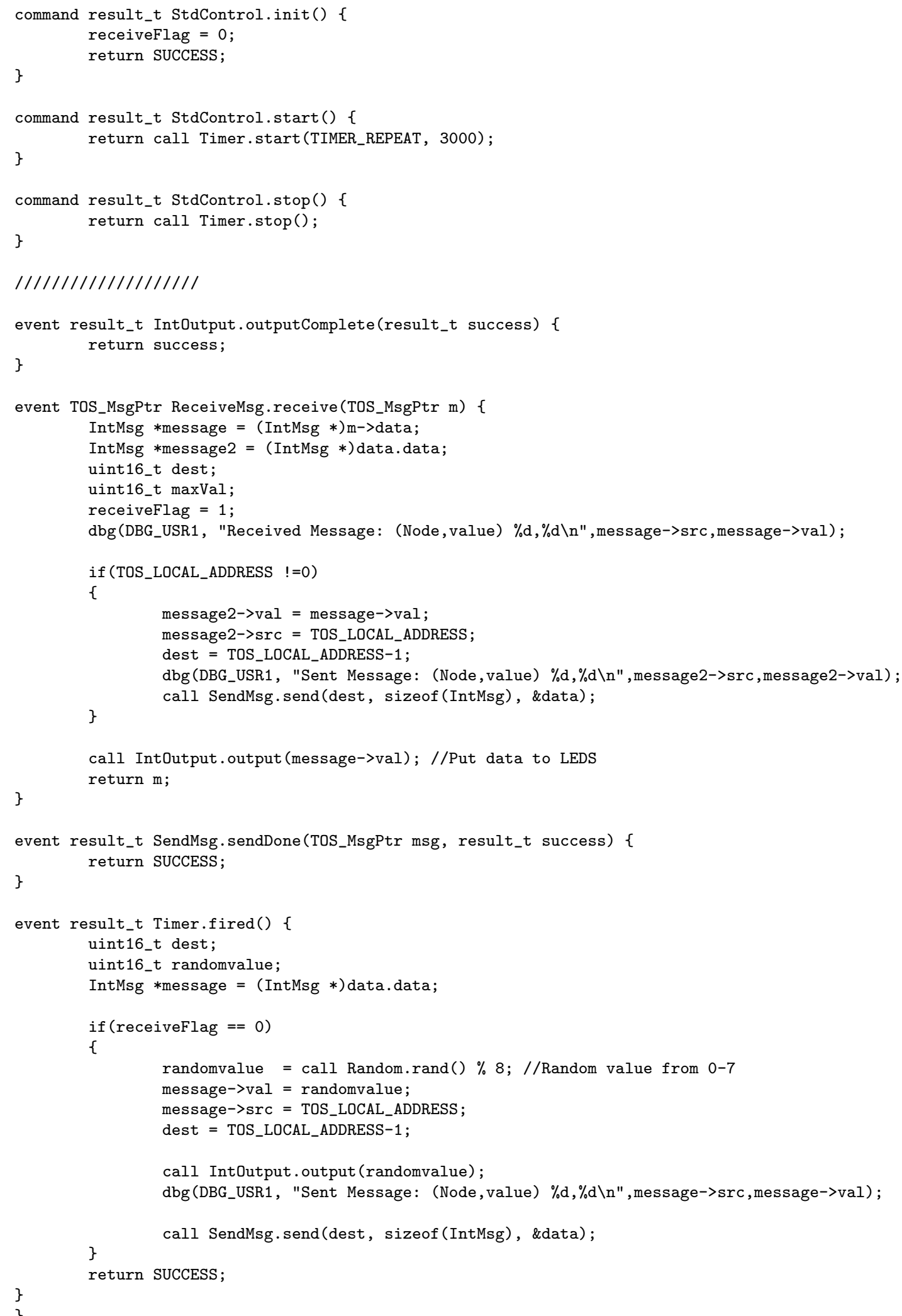




\section{2. $\quad$ Basic Tree Application}

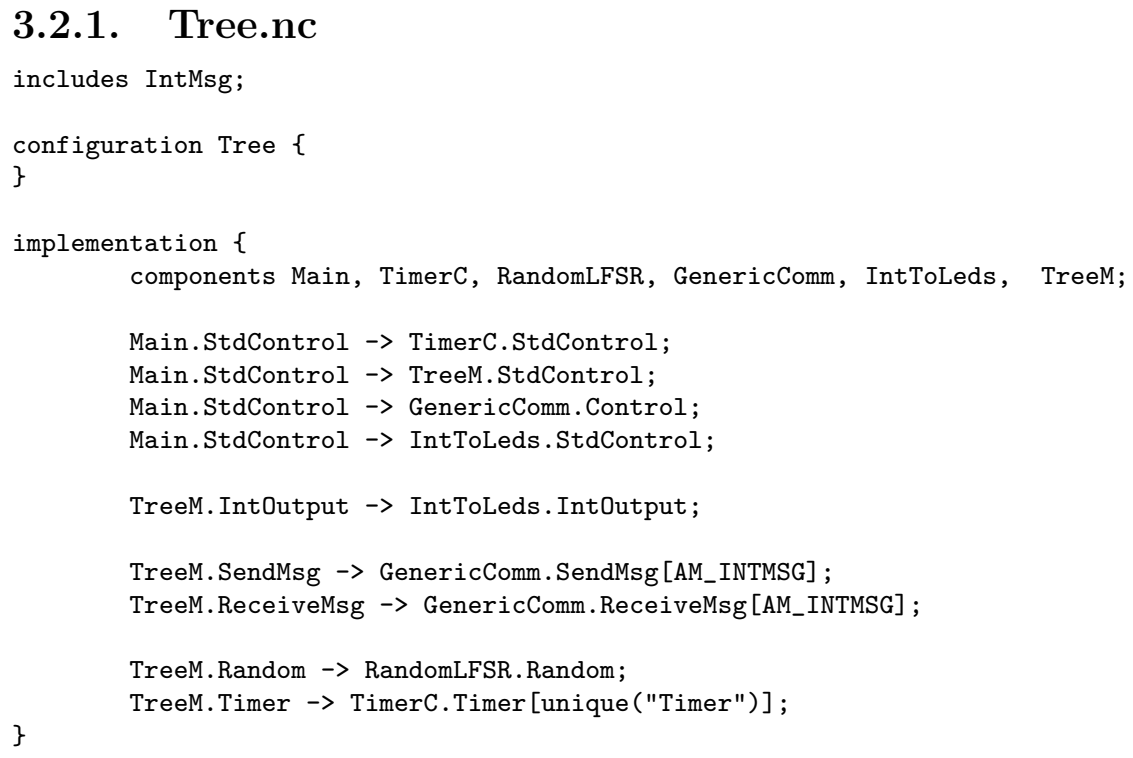

\subsubsection{TreeM.nc}

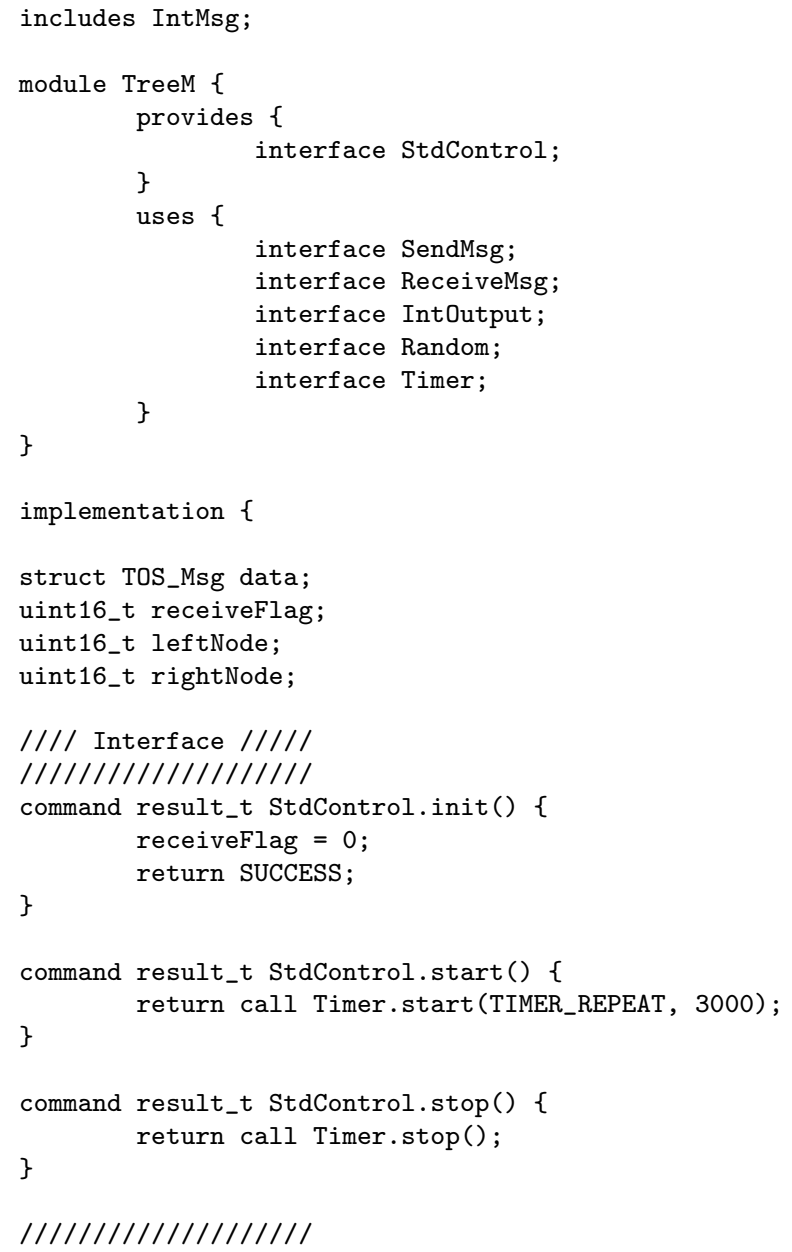




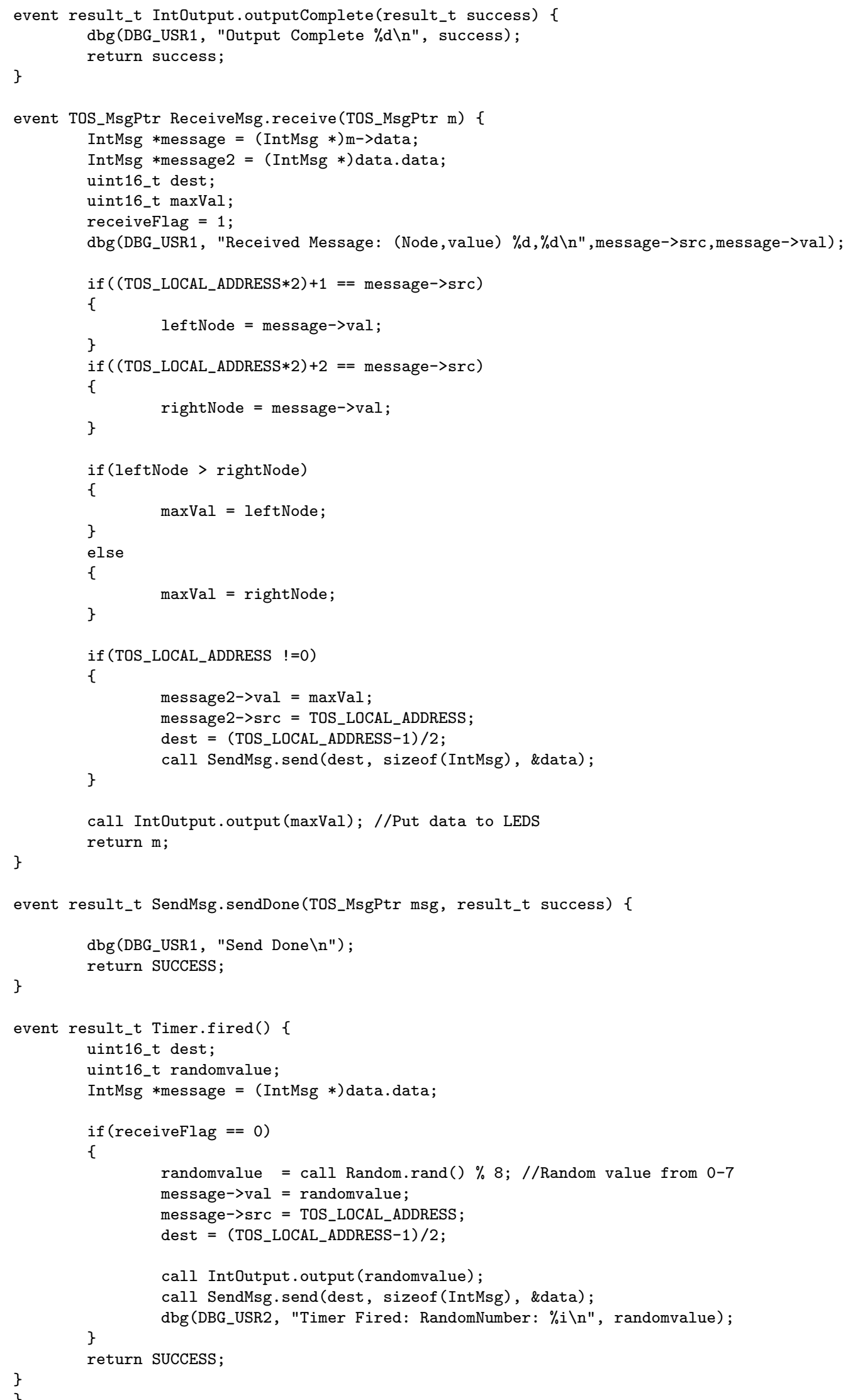




\subsection{Encrypted Tree Application}

\subsubsection{TreeTest.nc}

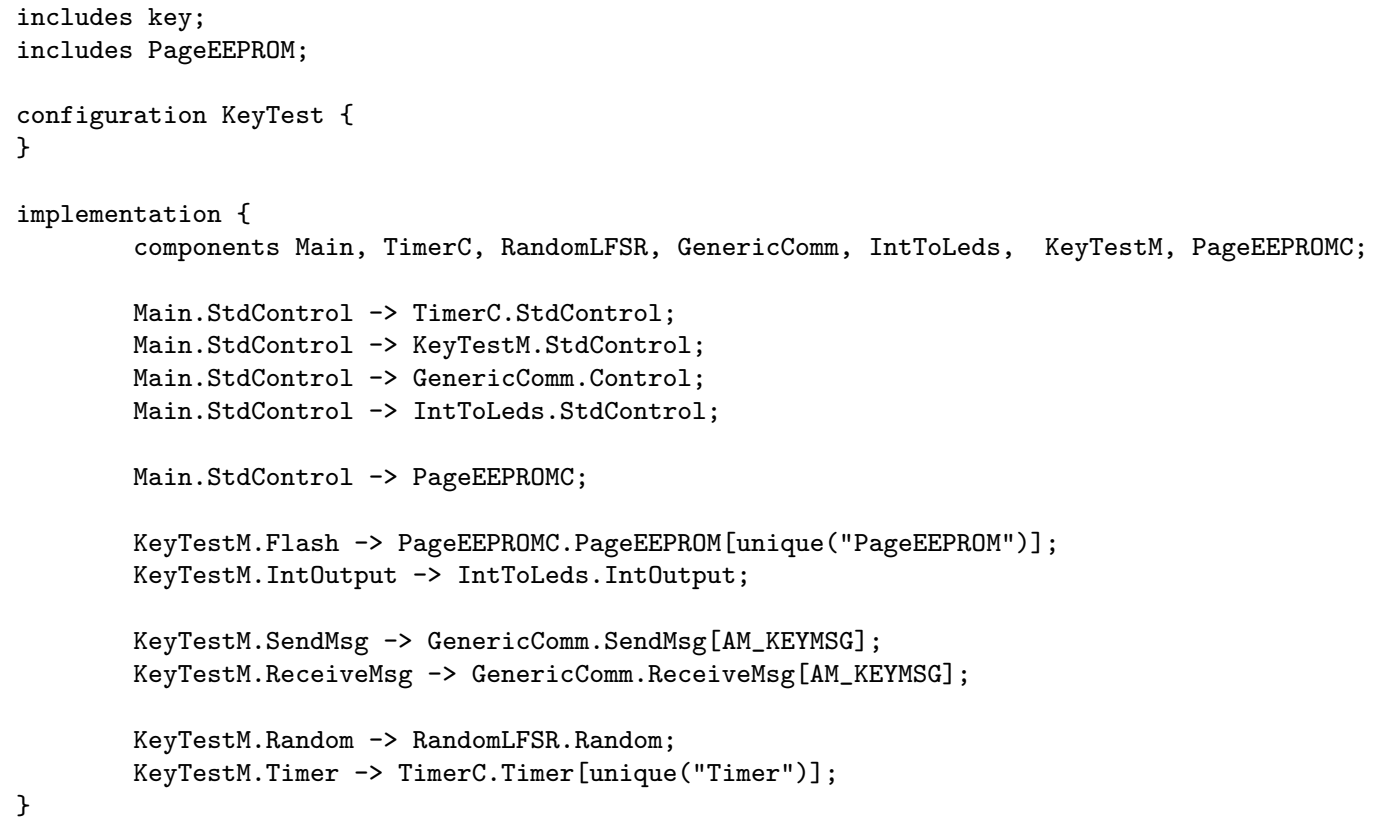

\subsubsection{TreeTestM.nc}

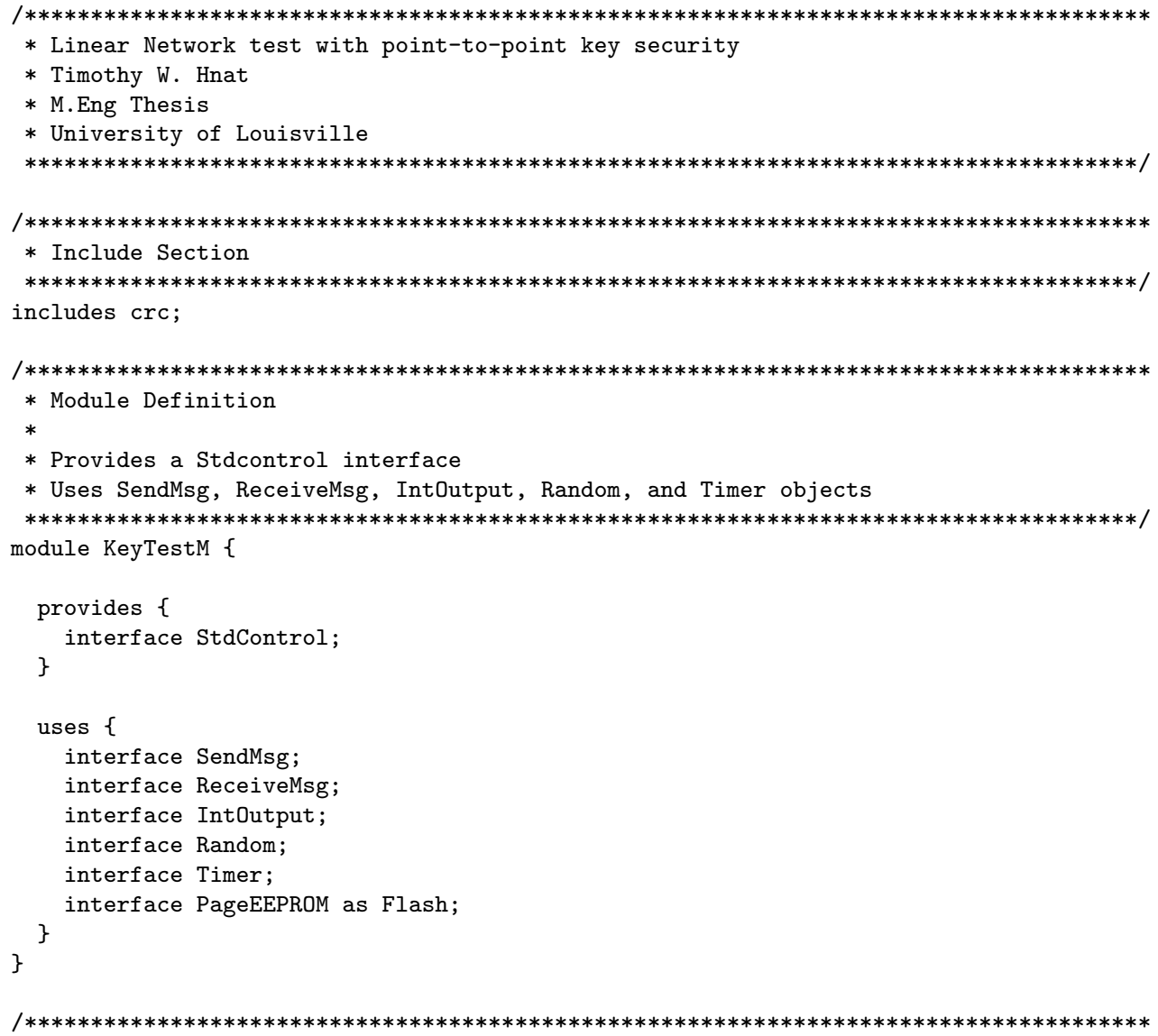




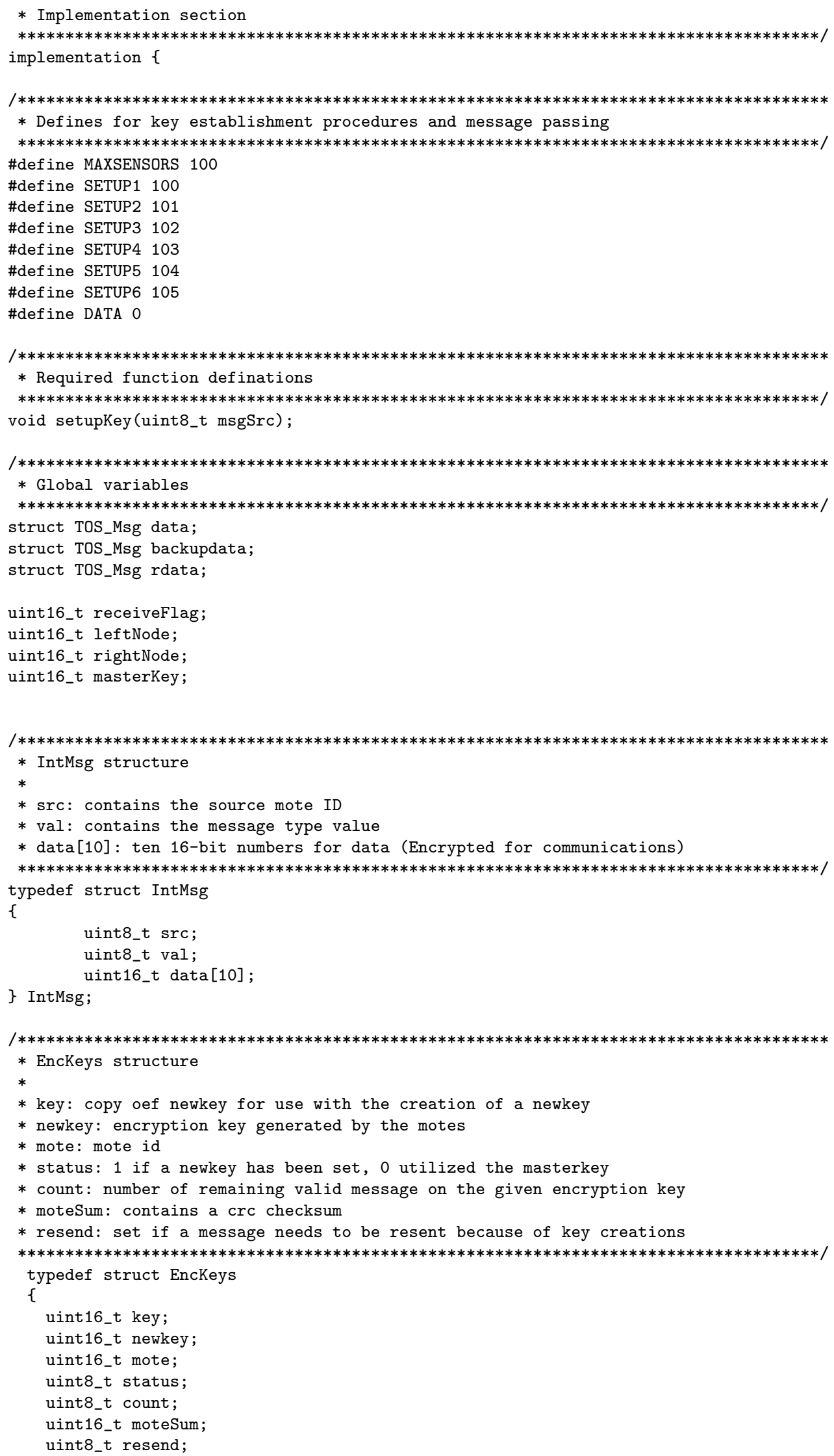


\} EncKeys;
EncKeys moteList [MAXSENSORS] ;

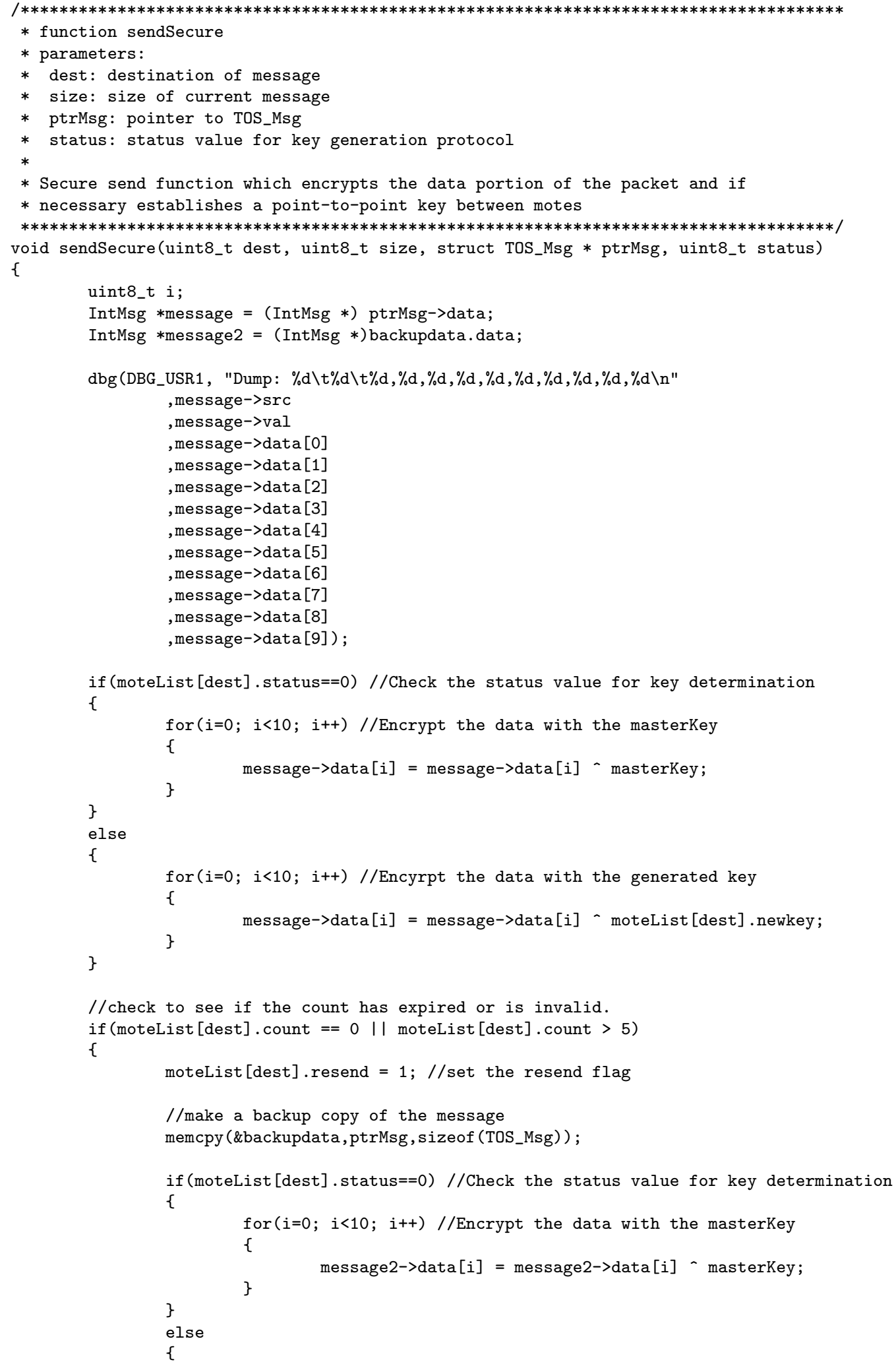




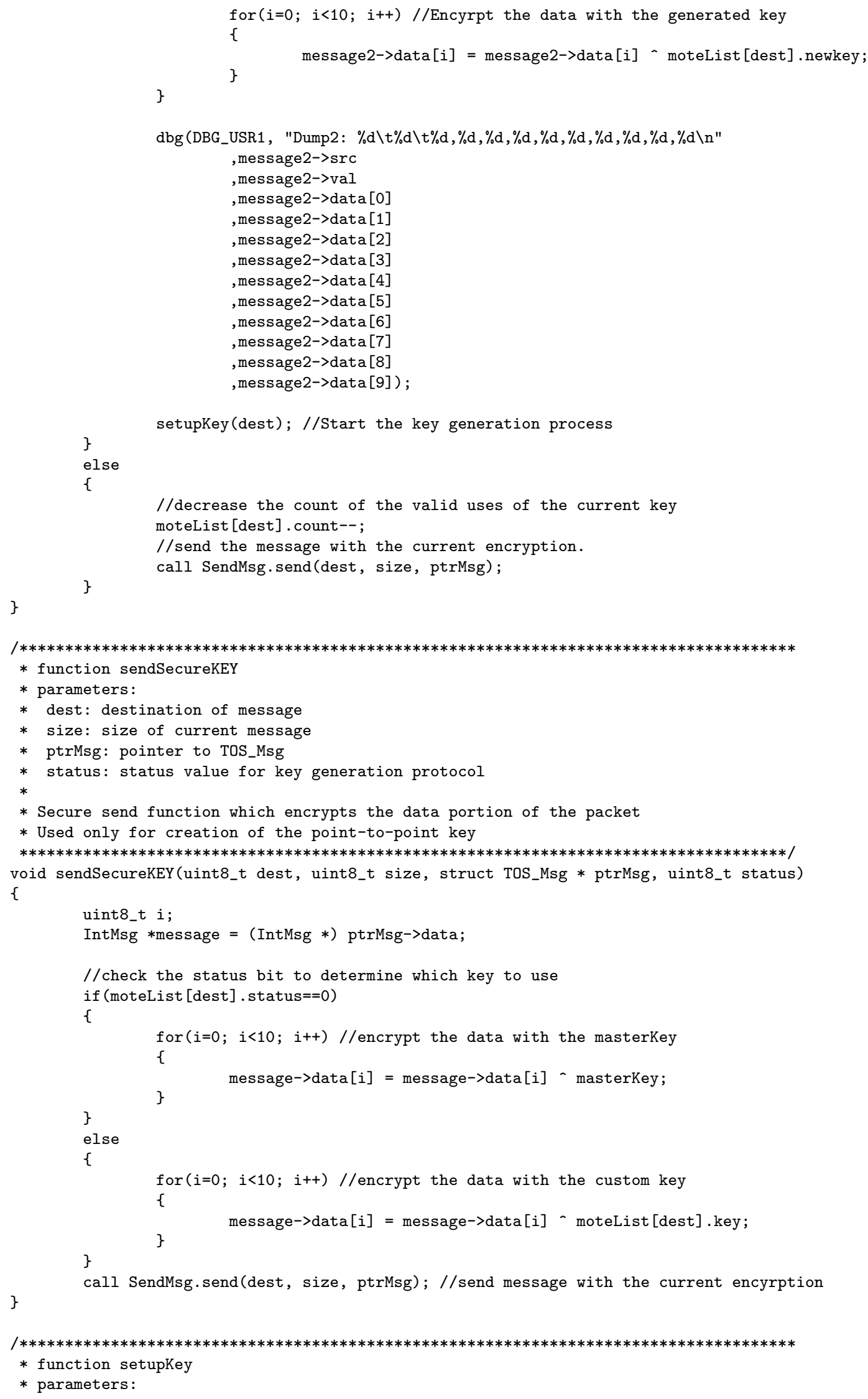




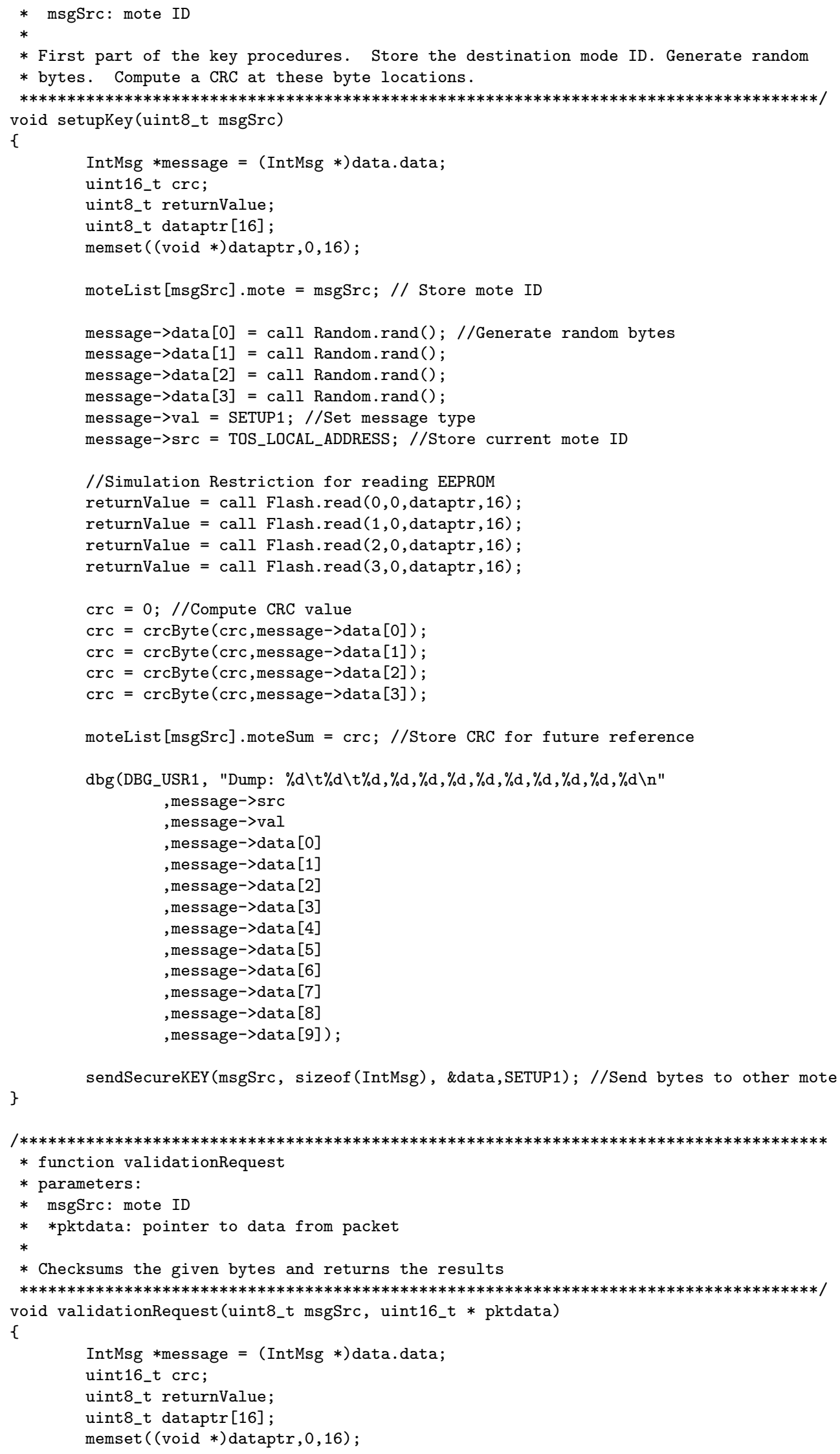




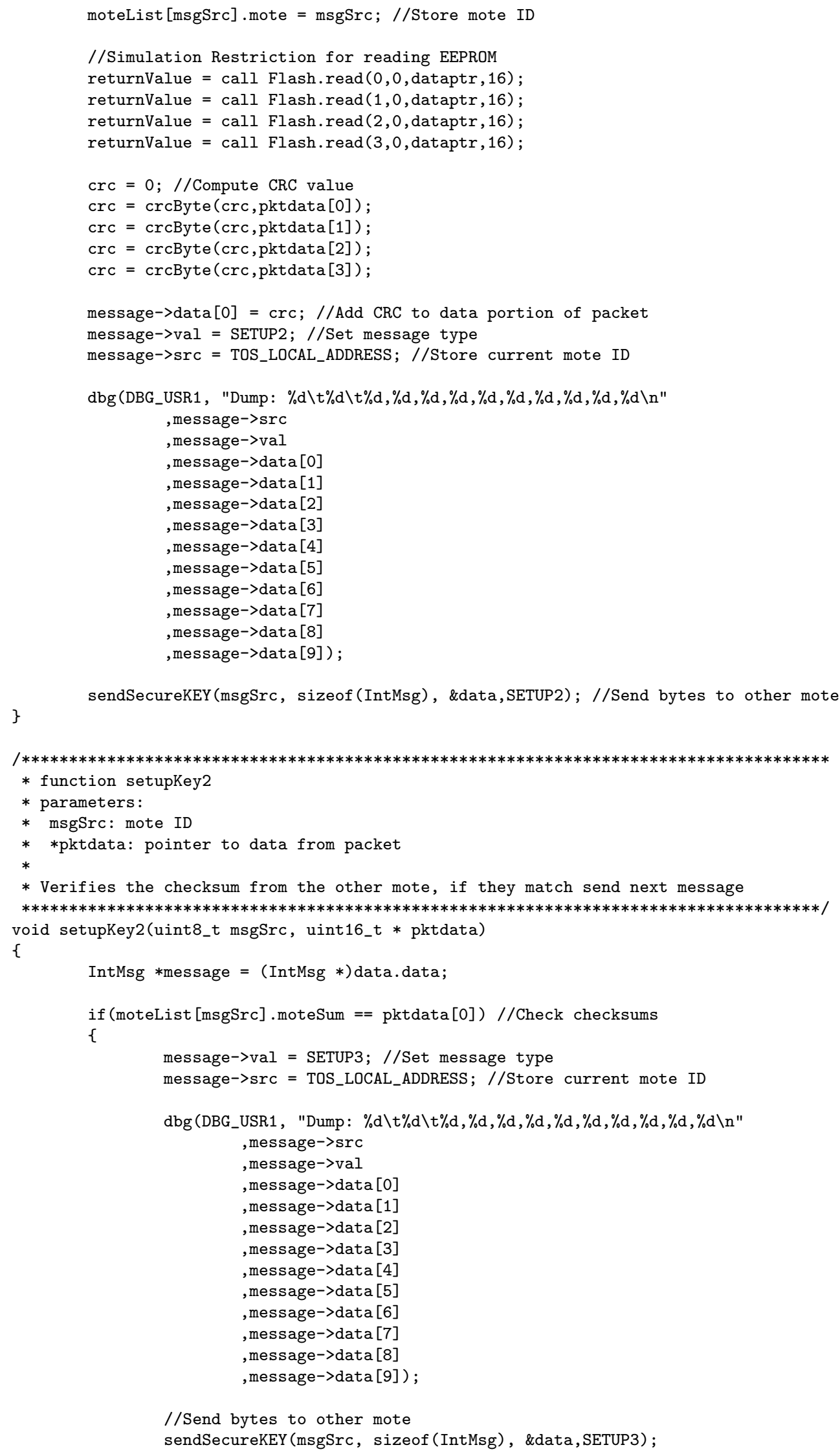

//Send bytes to other mote

sendSecureKEY (msgSrc, sizeof (IntMsg), \&data, SETUP3); 


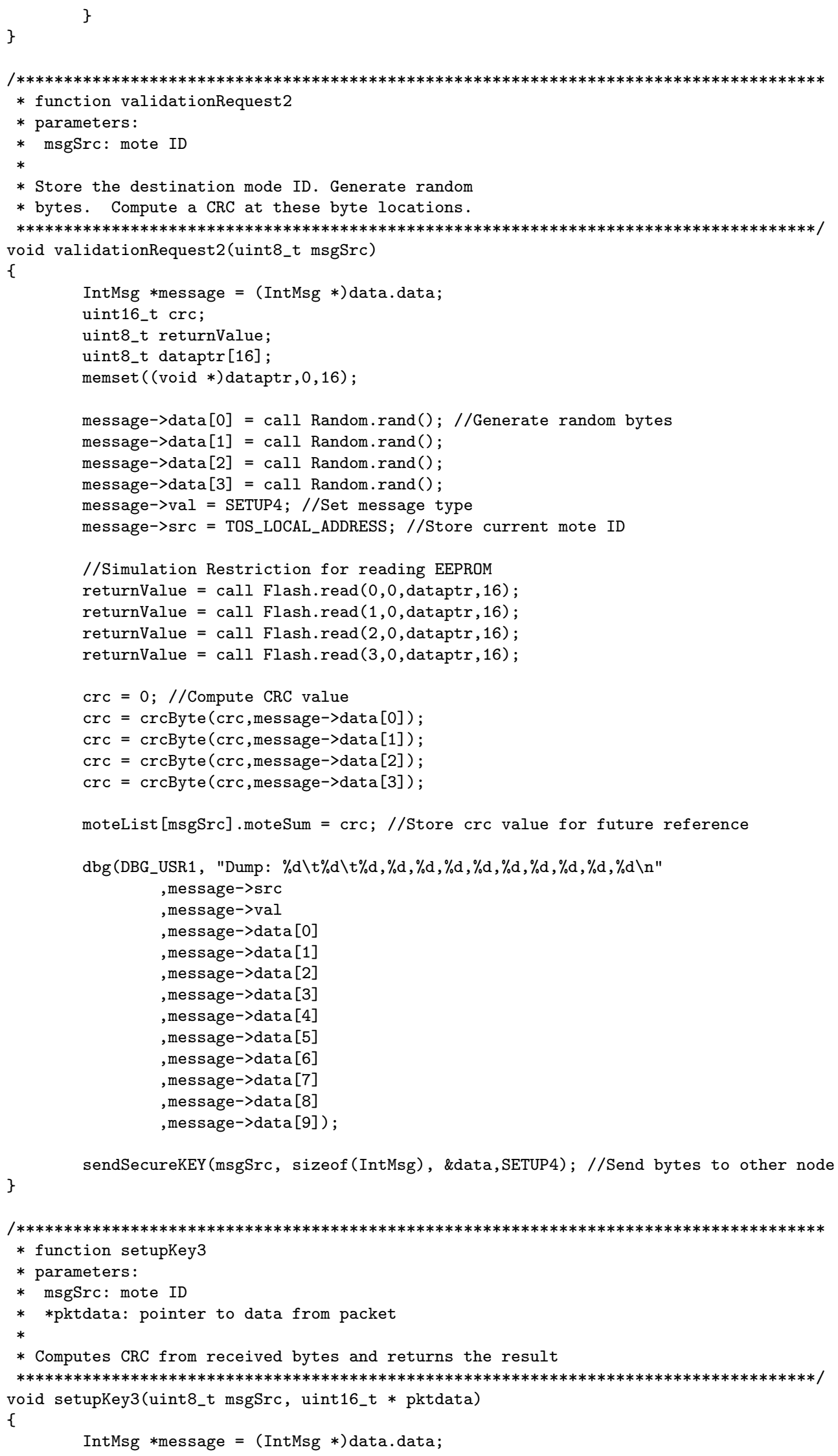




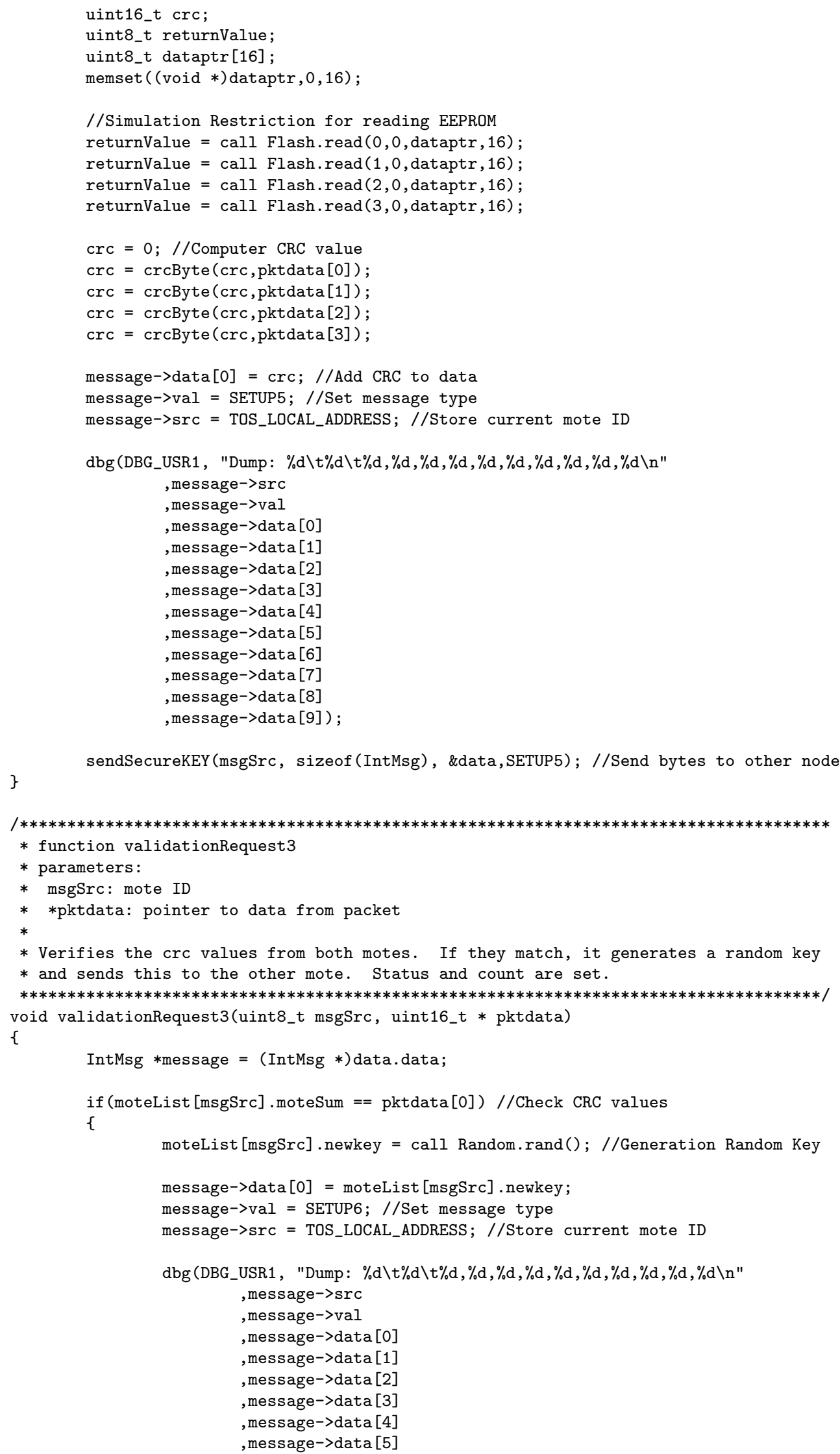




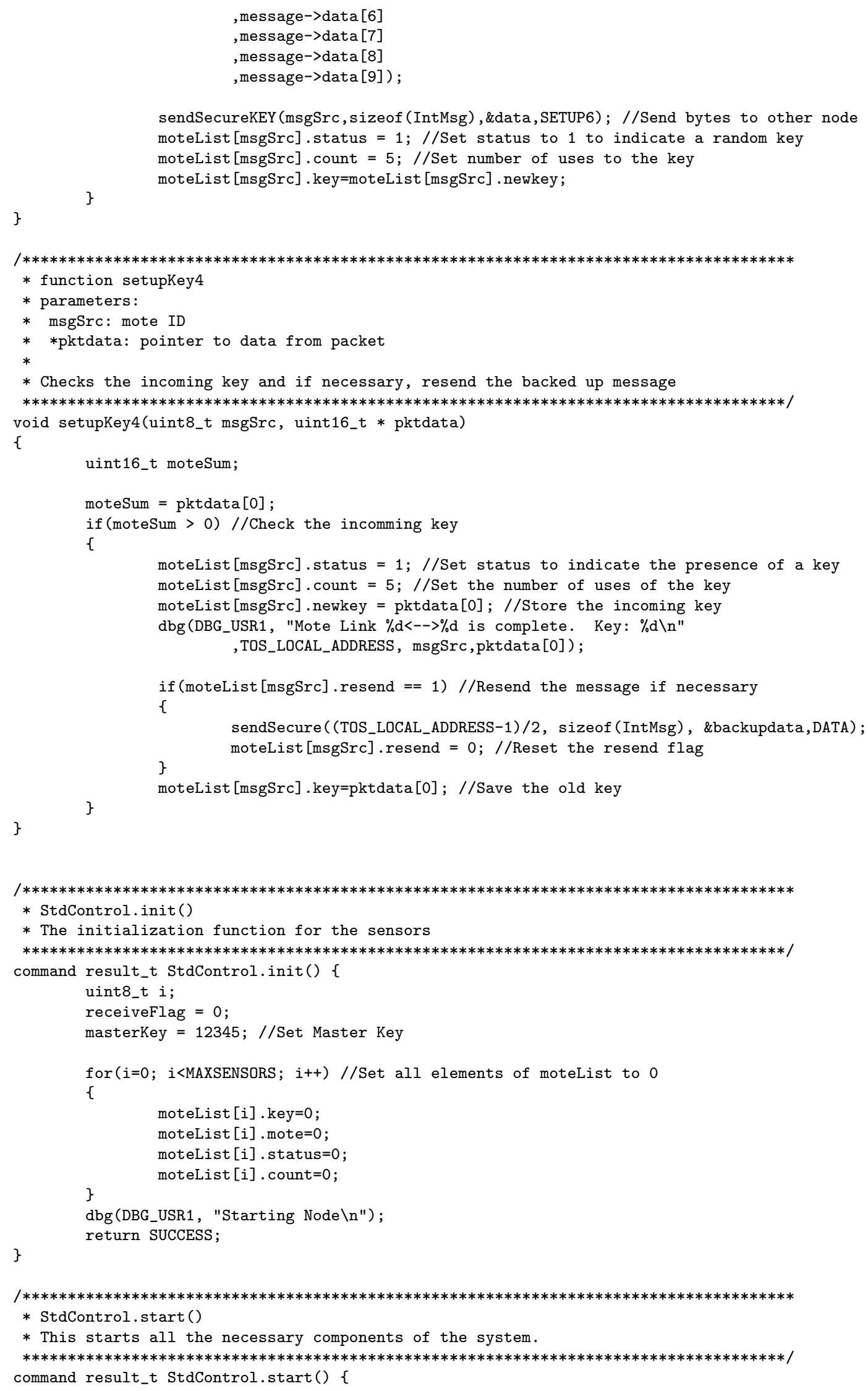




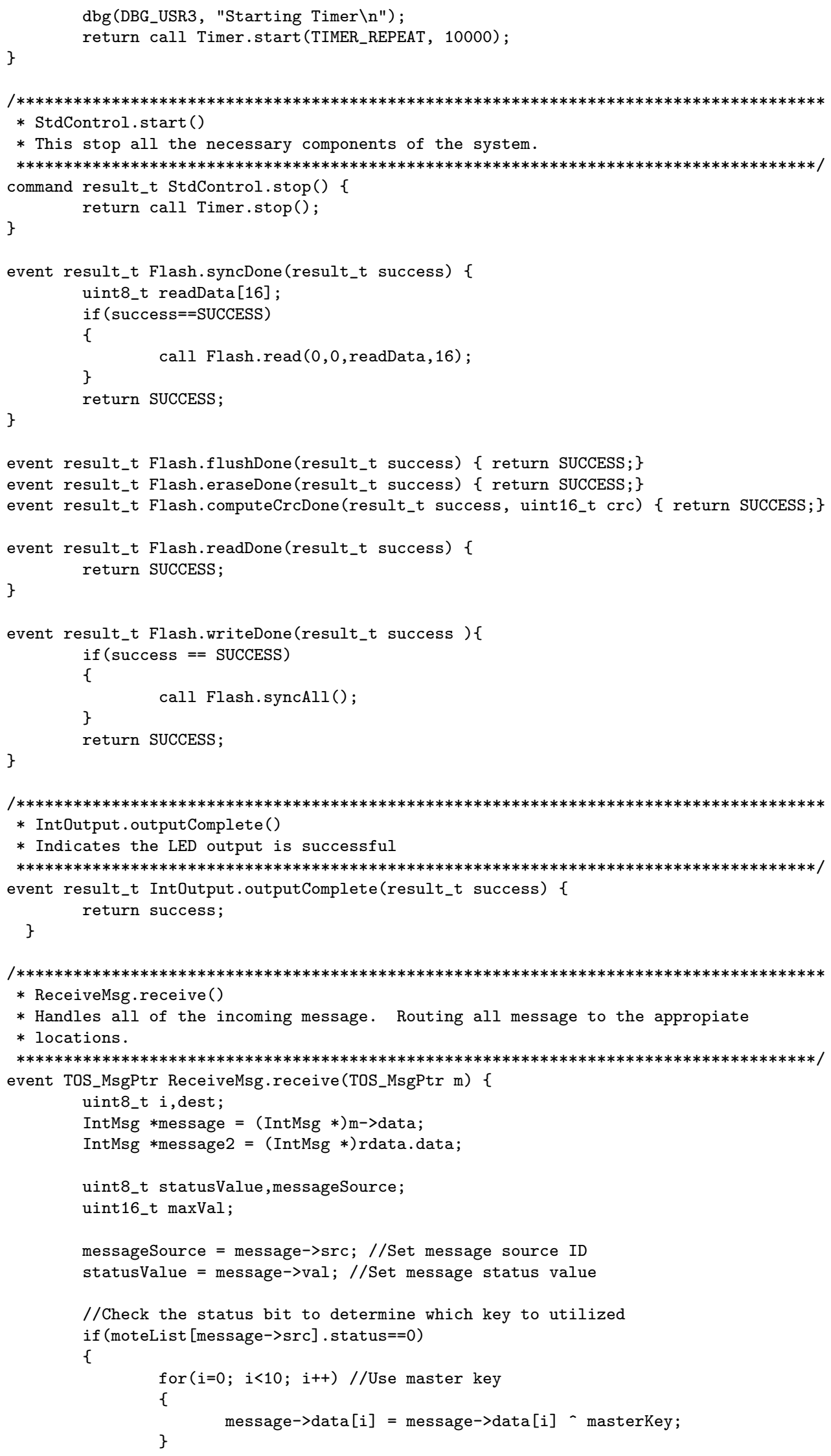




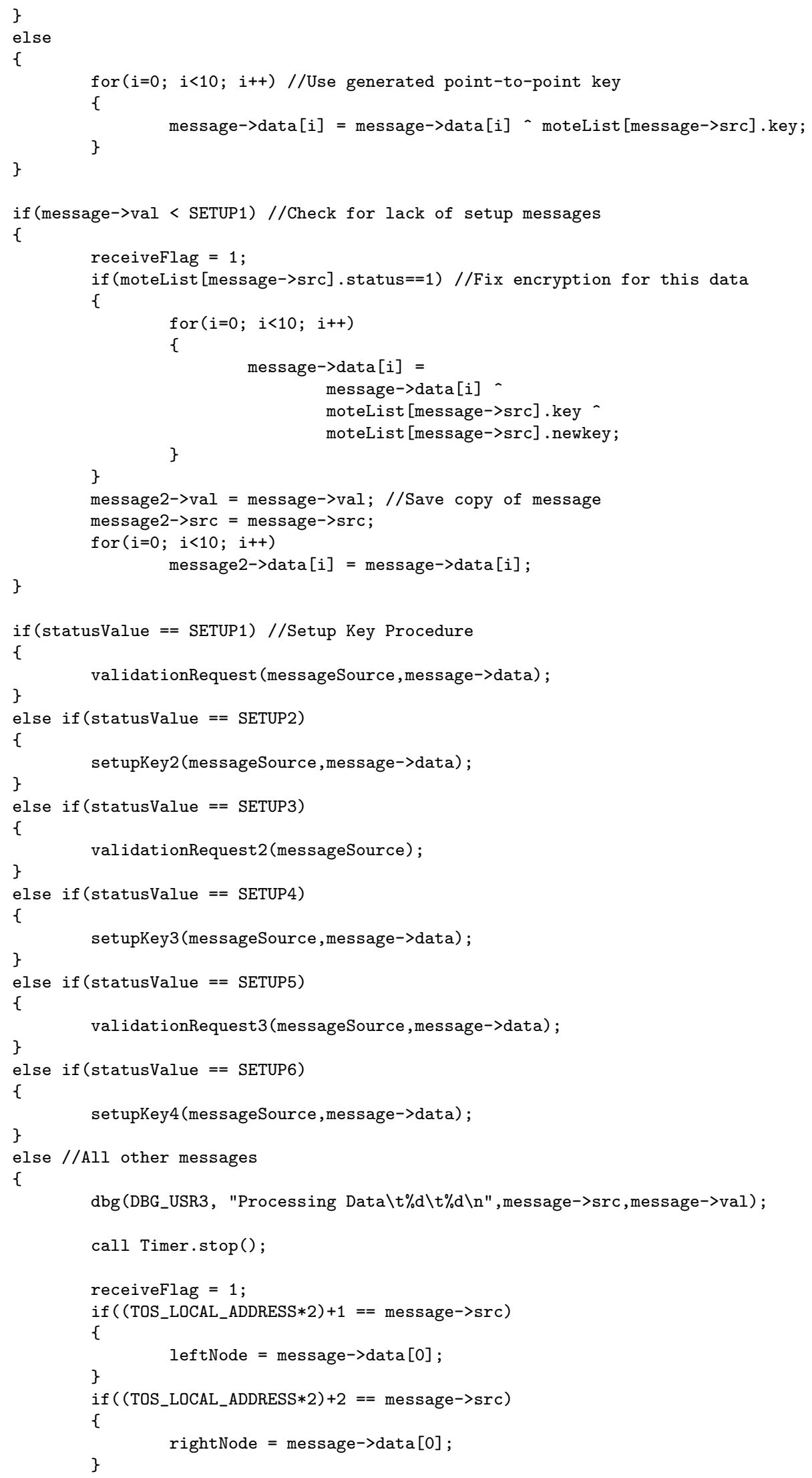




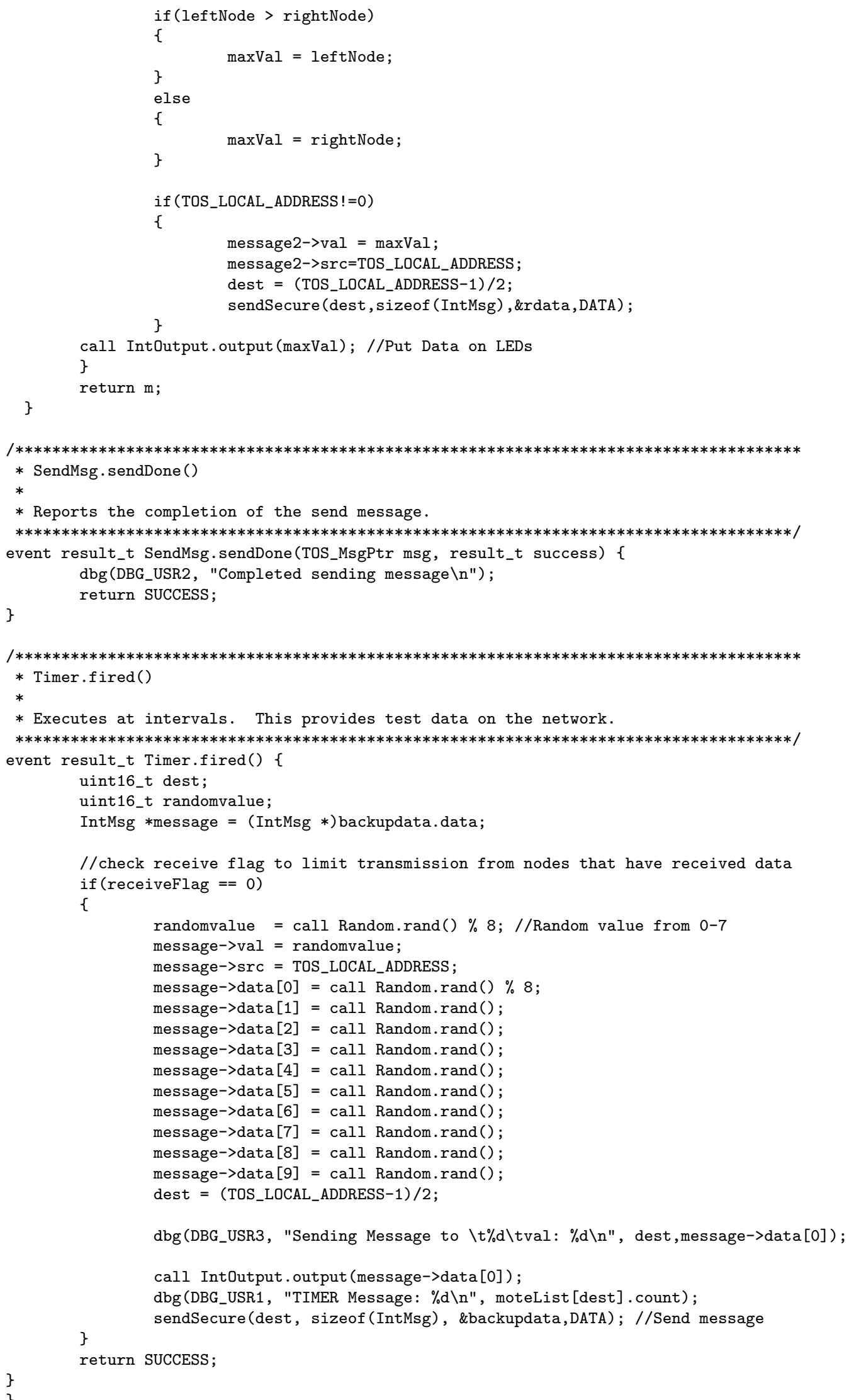

